\title{
The 5XFAD mouse model: a tool for genetic modulation of Alzheimer's disease pathology
}

\author{
PhD Thesis
}

\author{
in partial fulfilment of the requirements \\ for the degree "Doctor of Philosophy (PhD)" \\ in the Neuroscience Program \\ at the Georg August University Göttingen, \\ Faculty of Biology
}

submitted by

Sadim Jawhar

born in

Al-Bass, Lebanon 


\section{Declaration}

I hereby declare that the $\mathrm{PhD}$ thesis entitled "The 5XFAD mouse model: a tool for genetic modulation of Alzheimer's disease pathology" was written independently and with no other sources and aids than quoted.

Goettingen, 2011

Sadim Jawhar 


\section{PhD Thesis committee}

Prof. Dr. Thomas A. Bayer (Reviewer)

Department for Psychiatry

Division of Molecular Psychiatry

Georg August University

Goettingen

Prof. Dr. André Fischer (Reviewer)

Laboratory of Aging and Cognitive Diseases

European Neuroscience Institute

Goettingen

\section{Prof. Dr. Fred S. Wouters}

Department of Neuro-Sensory Physiology

Centre II; Physiology and Pathophysiology

Georg August University

Goettingen 


\section{Table of Contents}

\section{Acknowledgments}

Abbreviation list

1. Introduction

1.1 Alzheimer's disease

1.1.1 Epidemiology

1.1.2 Clinical features

.2 Neuropathological hallmarks

1.2.1 Amyloid plaques

1.2.2 Neurofibrillary tangles

1.2.3 Neuron loss and brain atrophy

1.2.4 Synaptic loss

1.2.5 Axonopathy

1.3 Diagnosis and treatment

1.3.1 Diagnosis

1.3.2 Treatment

1.4 The amyloid precursor protein

1.4.1 Non-amyloidogenic pathway

1.4.2 Amyloidogenic pathway

1.4.3 $\mathrm{A} \beta$ aggregation

1.5 The amyloid hypothesis

1.5.1 The intraneuronal amyloid hypothesis

1.6 Genetics

1.7 Mouse models of Alzheimer's disease

1.8 The 5XFAD mouse model

1.9 The pyroglutamate-modified $\mathrm{A} \beta$

1.9.1 Generation of pyroglutamate-modified $A \beta$

1.9.2 Biochemical properties of pyroglutamate-modified $\mathrm{A} \beta$

1.10 Glutaminyl cyclase

1.10.1 Physiological functions of glutaminyl cyclase

1.10.2 The role of glutaminyl cyclase in the generation of pyroglutamate-modified $A \beta$

2. Project I: "Characterization of the 5XFAD mouse model "

2.1 Overview of project I

2.2 Original publication

3. Project II: "Evaluating the role of glutaminyl cyclase and pyroglutamatemodified A $\beta$ in AD via the 5XFAD/hQC and 5XFAD/QC-KO mouse models"

3.1 Overview of project II

3.2 Original publication

4. Discussion

4.1 Project I: "Characterization of the 5XFAD mouse model"

4.1.1 The 5XFAD mouse model shows age-dependent neurological

deficits and motor impairments

4.1.2 The 5XFAD mouse model shows early pathological deficits in the spinal cord 
4.1.3 Neuron loss in 5XFAD mice correlates with the presence of intraneuronal $\mathrm{A} \beta$

4.1.4 Spatial working memory impairment in the 5XFAD mouse model

4.1.5 Reduced anxiety in 5XFAD mice

4.1.6 Plaque pathology and pyroglutamate-modified $A \beta$ in 5XFAD mice

4.1.7 Conclusions of project I

4.2 Project II: "Evaluating the role of glutaminyl cyclase and pyroglutamate-modified $\mathrm{A} \beta$ in $\mathrm{AD}$ via the 5XFAD/hQC and 5XFAD/QC-KO mouse models"

4.2.1 The 5XFAD/hQC mouse model

4.2.1.1 hQC overexpression alters pyroglutamate-modified A $\beta$ levels and plaque pathology

4.2.1.2 hQC overexpression worsens the behavior impairments of 5XFAD mice

4.2.2 The 5XFAD/QC-KO mouse model

4.2.2.1 Glutaminyl cyclase ablation reduces $A \beta$ concentration and plaque pathology in

5XFAD mice

4.2.2.2 Glutaminyl cyclase knock-out rescues the spatial working memory impairment in

5XFAD mice

4.2.3 Conclusions of project II 
"I graduate and I fail to find a wall on which to hang my certificate" Mourid Al-Bargouthi-Palestenian Poet

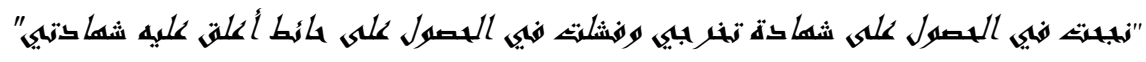

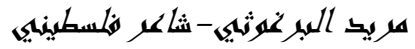




\section{Acknowledgments}

I gratefully acknowledge the continuous support, encouragement and insightful guidance of Prof. Dr. Thomas A. Bayer who gave me the chance to work under his supervision and has been following my work throughout the preparation of this thesis.

I also wish to express my gratitude to Prof. Dr. André Fischer and Prof. Dr. Fred S. Wouters for agreeing to be members of my PhD committee and for their time and insightful comments.

Dr. Oliver Wirths deserves special thanks for all the help, patience, advice and inspiring comments he offered me during my thesis preparation.

I would also like to thank Prof. Dr. Michael Hoerner and Sandra Drube for the great assistance they provided me ever since I applied to the program.

Additional thanks go to all the former and current members of Prof. Bayer's lab: Marie-Caroline Cotel, Andrea Marcello, Vivek Venkataramani, Katharina Dietrich, and Yvonne Bouter.

My thanks also go to Anika Saul for all the tips in biochemistry and in the running of necessary computer programs.

I owe a special dept of thanks to Ditte Z. Christensen for her continuous help and invaluable advice about work and life in general, even after she left the lab.

I am also indebted to Petra Tucholla for always being available for technical and logistic support, and for cheering me up especially when computer technology insisted on failing me.

Special thanks go to Anna Trawicka for the work she accomplished during her bachelor thesis, and more importantly for being such an unforgettable friend.

The past three years would not have been the same without the girls of the front office, with whom I shared moments of joy and disappointments in and out of Goettingen. Antje Hillmann, "my beautiful German friend in green", deserves deep gratitude for her endless emotional support and continuous help in overcoming technical, bureaucratic and German language obstacles. My dear friend Jessica L. Wittnam deserves special thanks for keeping me sane through her unfailing support and views on science and life, and for being my English language referee.

Many thanks should also go to Alwaleed Alkhaja, who never let me down, for being who he is and for always being there to put a smile on my face; last but not least, for proofreading my thesis and providing me with insightful comments.

More and above, I owe a special gratitude to Moslih and Samia Kanaaneh from Palestine who stood by me in every step, shared my dreams and gave me a hope in a better future despite the distance; knowing you was my most important experiment.

I can hardly find words to express my heartfelt gratitude and sense of indebtedness to my parents, Hussein Jawhar and Zahra Al-Husseini, who have never lost their unconditional belief in me and my capacity to succeed, even at moments in which I myself lost that belief. They patiently supported my decisions and were always willing to give me much more than I expected. To you, I dedicate my thesis as an embodiment of your success. 


\section{List of Abbreviations}

5XFAD Five familial AD mutations

AD

Alzheimer's disease

ADAM A disintegrin and metalloproteinase

AICD APP intracellular domain

APH-1 Anterior pharynx-defective-1

APLP Amyloid precursor like protein

APOE Apolipoprotein E

APP Amyloid precursor protein

$\mathbf{A} \boldsymbol{\beta}$

Amyloid beta

$\mathbf{A} \boldsymbol{\beta}_{1-\mathbf{x}} \quad \mathrm{A} \beta$ of variable $\mathrm{C}$-terminal lengths, starting with unmodified $\mathrm{N}$-terminal

$\mathbf{A} \boldsymbol{\beta}_{\mathbf{p E 1 1 - x}} \quad \mathrm{A} \beta$ of variable $\mathrm{C}$-terminal lengths, starting with pyroglutamate at the eleventh position

$\mathbf{A} \boldsymbol{\beta}_{\mathbf{p E 3} \text {-x }} \quad \mathrm{A} \beta$ of variable $\mathrm{C}$-terminal lengths, starting with pyroglutamate at the third position

$\mathbf{A} \boldsymbol{\beta}_{(\mathbf{E}) 11-\mathbf{x}} \quad \mathrm{A} \beta$ truncated at the eleventh position, starting with glutamate

$\mathbf{A} \boldsymbol{\beta}_{(\mathbf{E}) 3-\mathbf{x}} \quad \mathrm{A} \beta$ truncated at the third position, starting with glutamate

$\mathbf{A} \boldsymbol{\beta}_{(\mathbf{Q}) 3-\mathbf{x}} \quad \mathrm{A} \beta$ truncated at the third position, starting with glutamine

$\mathbf{A} \boldsymbol{\beta}_{\mathbf{x}-40} \quad \mathrm{~A} \beta$ ending with amino acid 40

$\mathbf{A} \boldsymbol{\beta}_{\mathbf{x}-42} \quad \mathrm{~A} \beta$ ending with amino acid 42

BACE Beta site APP-cleaving enzyme

CA Cornu Ammonis

CAA Congophilic amyloid angiopathy

CNS Central nervous system

CTFa C-terminal fragment generated by $\alpha$-secretase

CTF $\boldsymbol{C}$-terminal fragment generated by $\beta$-secretase

ER Endoplasmic reticulum

FAD Familial AD

GnRH Gonadotropin releasing hormone

GSK-3 Glycogen synthase kinase 3

hQC Human QC

imAPP Immature APP

LTP Long term potentiation

MAPK Mitogen-activated protein kinase

mAPP Mature APP

MAPs Microtubule associated proteins

MCI Mild cognitive impairment

MCP Monocyte chemotactic protein

MMSE Mini mental state examination

mQC Murine QC 


$\begin{array}{ll}\text { MRI } & \text { Magnetic resonance imaging } \\ \text { MVB } & \text { Multivesicular bodies } \\ \text { NFT } & \text { Neurofibrillary tangles } \\ \text { NMDA } & \text { N-methyl D-aspartate } \\ \text { PEN-2 } & \text { Presenilin enhancer-2 } \\ \text { PHFs } & \text { Paired helical filaments } \\ \text { PS } & \text { Presenilin } \\ \text { QC } & \text { Glutaminyl cyclase } \\ \text { QC-KO } & \text { QC homozygous knock-out } \\ \text { sAPPa } & \text { Soluble APP fragment generated by } \alpha \text {-secretase } \\ \text { SAPP } \beta & \text { Soluble APP fragment generated by } \beta \text {-secretase } \\ \text { TGN } & \text { trans-Golgi network } \\ \text { TRH } & \text { Thyrotropin releasing hormone } \\ \boldsymbol{\alpha} \text {-secretase } & \text { Alpha secretase } \\ \boldsymbol{\beta} \text {-secretase } & \text { Beta secretase } \\ \boldsymbol{\gamma} \text {-secretase } & \text { Gamma secretase }\end{array}$




\section{Introduction}

\subsection{Alzheimer's disease}

When Alois Alzheimer presented the case of his patient Auguste Deter at the Tuebingen meeting of the Southwest German Psychiatrists in 1906, he did not attract much attention or stimulate any discussion in the audience. The young doctor likely would not have believed that, 100 years later, the disease that now holds his name would be the most common cause of dementia and a source of critical medical and economical problems. At this meeting, Alzheimer presented Auguste Deter's symptoms and reported the histopathological features that are now associated with Alzheimer disease (AD): neuron loss, extracellular amyloid plaques and intracellular neurofibrillary tangles (Dahm, 2006).

\subsubsection{Epidemiology}

According to the World Alzheimer Report 2010, there were 35.6 million people worldwide living with dementia in 2010 (World Alzheimer Report, 2010). This number will increase to 65.7 million by 2030 and 115.4 million by 2050 . Around two-thirds of current patients live in low and middle-income countries, where the sharpest increase in numbers is set to occur. AD accounts for 50-60\% of the dementia cases (Ferri et al., 2005). The number of people suffering from dementia in Germany is approximately 1.2 million; in which AD accounts for two thirds of cases (Deutsche Alzheimer Gesellschaft, 2010). In Lebanon, there is an estimated number of 30,000 AD cases and an estimate of 1.5 million cases in Arab countries (Alzheimer's Association Lebanon, 2011).

Life expectancy for patients with AD can vary from 3 to 10 years (Zanetti et al., 2009). According to the World Health Organization report 2011, AD and other forms of dementias represent the fourth highest cause of death in high-income countries, accounting for $4.1 \%$ of the total deaths. The total estimated worldwide costs of dementia were 604 billion US dollars in 2010 (World Health Organization report, 2011, World Alzheimer Report, 2010). 


\subsubsection{Clinical features}

$\mathrm{AD}$ is a slowly progressive disorder manifested by many symptoms that affect memory in addition to other cognitive and behavioral abilities of the patients. Prior to the appearance of clinical symptoms, neurodegeneration starts and accumulates until it reaches a certain threshold, during which neuropsychological tests can reveal mild cognitive impairments (MCI) (Linn et al., 1995). MCI is defined by cognitive decline greater than what is expected for an individual's age and education level, but which does not interfere notably with daily life activities. At this stage, the differentiation between incipient AD, a reversible condition, normal aging or other forms of dementia is unreliable (Gauthier et al., 2006).

The amnesic subtype of MCI exhibits a high risk of progression to AD and thus can be considered a predemential stage of the disease (Petersen, 2004). The predementia stage of the disease starts with impairments in episodic memory, which is represented by difficulties with recent memories. In addition, subtle effects on other cognitive functions start to appear; this may include planning, attention, flexibility, abstract thinking and verbal ability (Mega et al., 1996; Forstl and Kurz, 1999). In the early stages of the disease, the deterioration in the recent form of the declarative memory continues, in addition to vocabulary shrinkage, spatial disorientation, depression and apraxia (Moore and Wyke, 1984; Burns et al., 1990; Locascio et al., 1995; Trobe et al., 1996). As the disease progresses, the patients require close supervision due to the loss of daily life skills, worsening of vocabulary, hallucinations and delusions, aggression, irritability and wandering (Mega et al., 1996; Forstl and Kurz, 1999). At the terminal stages of the disease, all forms of memory including early memories are usually severely impaired. Besides, patients may develop some motor impairments such as poor facial expression, rigidity, posture/gait and bradykinesia (Scarmeas et al., 2004). Some AD patients show epileptic seizures as well (Romanelli et al., 1990).

\subsection{Neuropathological hallmarks}

\subsubsection{Amyloid plaques}

The amyloid plaques are spherical and complex protein accumulations with $\beta$-sheet structure. They can be divided into neuritic plaques and diffuse plaques (Yamaguchi et al., 1988b; Small, 1998). Neuritic plaques represent one of the major hallmarks of AD. They 
consist of foci of extracellular amyloid beta (A $\beta$ ) peptides associated with axonal and dendritic enlargements that contain degenerated mitochondria, organelles and lysosomes (dystrophic neurites). Microglia frequently exist within and adjacent to the amyloid core, whereas astrocytes surround the plaque and send their processes towards it. A cross section of a neuritic plaque varies from 10 to $120 \mu \mathrm{m}$ in size and the densities of
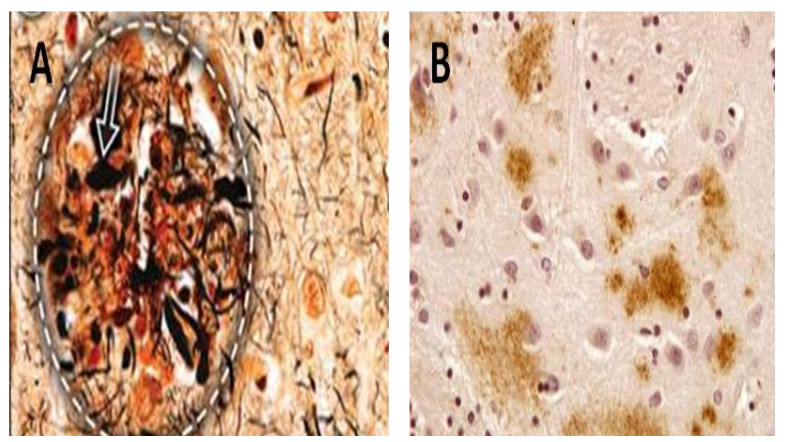

Fig 1. Amyloid plaques visualized by immunohistochemistry. A) A neuritic plaque with dystrophic neurites (small arrow). Adapted from (Holtzman et al., 2011). B) Diffuse plaques. Adapted from (Duyckaerts et al., 2009).

amyloid fibrils vary among plaques as well. In addition to $\mathrm{A} \beta$, neuritic plaques in $\mathrm{AD}$ patients contain many other proteins such as Tau, amyloid precursor protein (APP) and ubiquitin (Su et al., 1998; Norfray and Provenzale, 2004)

On the other hand, diffuse plaques vary in size from $50 \mu \mathrm{m}$ to several hundred $\mu \mathrm{m}$ and consist of amorphous, non-fibrillar plaques of $\mathrm{A} \beta$ with little or no detectable neuritic dystrophy (Yamaguchi et al., 1988a) (Fig. 1). Therefore, they were suggested to represent precursors in neuritic plaque generation (Dickson, 1997).

\subsubsection{Neurofibrillary tangles}

In contrast to the extracellular amyloid plaques, neurofibrillary tangles (NFTs) consist of large non-membranous bundles of abnormal fibers present in the perikarya or in the apical dendrites (Fig. 2). Using electron microscopy, it was revealed in the early 1960's that NFTs consist mostly of $10 \mathrm{~nm}$ filament pairs wound into paired helical filaments (PHFs) (Kidd, 1963). It took around three decades to confirm the composition of these PHFs to be hyperphosphorylated tau protein (Grundke-Iqbal et al., 1986; Lee et al., 1991). Tau protein is a member of the microtubule-associated proteins family (MAPs) that is encoded by a single gene on chromosome 17 containing 16 exons (Neve et al., 1986). Alternative splicing of these exons

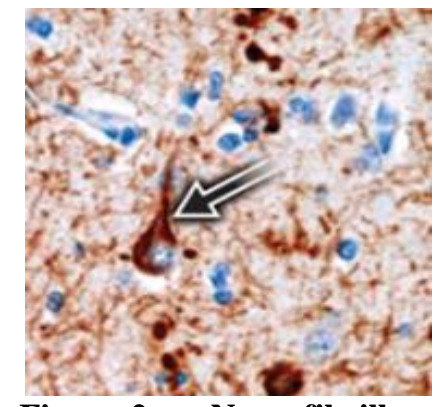

Fig. 2. Neurofibrillary tangles in AD. Antiphospho-tau antibody reveals hyperphosphorylated tau accumulation in neuronal cell bodies (large arrow). Adapted from (Holtzman et al., 2011) 
produce the six isoforms that are present in the human brain (Goedert et al., 1989). Tau is mainly expressed in neurons of the central nervous system (CNS) where it is primarily located in the axons and absent from the dendrites. It interacts with tubulin in order to promote its assembly into microtubules and to stabilize microtubules; therefore, it is important for stabilizing axons (Hirokawa et al., 1996). However, hyperphosphorylation of tau leads to its dissociation from microtubules and its subsequent aggregation into PHFs (Alonso et al., 2001). Several kinases have been suggested to be involved in phosphorylating tau at various sites such as glycogen synthase kinase 3 (GSK-3) (Mandelkow et al., 1992), cyclin-dependent kinase 5 (CDK-5) (Baumann et al., 1993) and mitogen-activated protein kinase (MAPK) (Drewes et al., 1992).

Although NFTs are widely considered to be downstream of amyloid pathology, they represent a better correlate with clinical and cognitive stages in $\mathrm{AD}$ patients in comparison to the neuritic plaques, and they are thus widely used for postmortem staging of AD (Braak and Braak, 1991).

\subsubsection{Neuron loss and brain atrophy}

In addition to amyloid plaques and NFTs, neuronal loss in some cortical and subcortical regions represents a main pathological feature of the disease. During the progression of the disease, the persistent neuron loss leads to atrophy in various brain regions such as the hippocampus, entorhinal cortex, and nucleus basalis of Meynert. Using unbiased stereological techniques, it was shown that significant neuron loss was detectable in the entorhinal cortex in early subtle cases of AD (Gomez-Isla et al., 1996). No neuron loss was reported in the hippocampus of preclinical AD cases, whereas significant neuronal loss was detected in the CA1, hilus and subiculum of AD cases (West et al., 2004). The nucleus basalis of Meynert is a major source of cholinergic innervations for the cerebral cortex and is one of the first brain

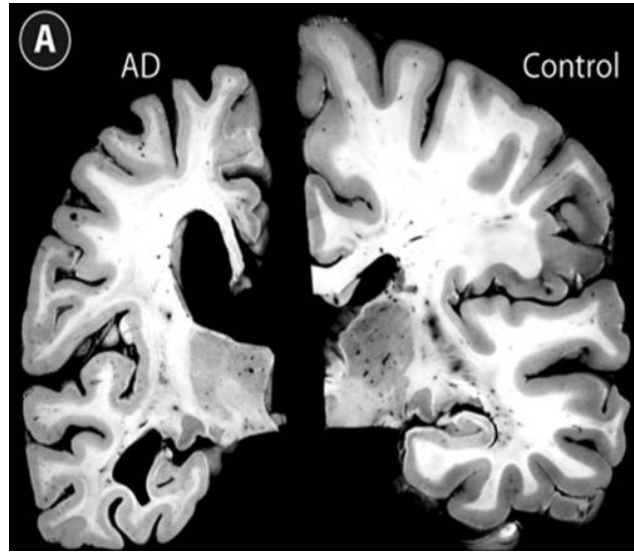

Fig. 3. Brain atrophy in AD. Postmortem brain section from an $\mathrm{AD}$ case (left) compared with cognitively normal individual (right) reveals severe brain atrophy in $\mathrm{AD}$. Adapted from (Holtzman et al., 2011). 
regions discovered with profound neuron loss (Davies and Maloney, 1976; Cullen et al., 1997). In addition, neuronal death has been reported in other brain regions such as the anterior olfactory bulb (ter Laak et al., 1994), amygdala (Vereecken et al., 1994), lower and medial parts of substantia nigra (Uchihara et al., 1992), nucleus coeruleus (Busch et al., 1997) and raphe nuclei (Aletrino et al., 1992) of AD patients.

In addition to the microscopic pathology mentioned above, $\mathrm{AD}$ brain exhibits pronounced macroscopic atrophy leading to widening of the sulci, shrinkage of the gyri and enlargement of the ventricles (Fig. 3). This atrophy is represented by reduced weight in addition to volumetric reduction that can be detected by magnetic resonance imaging (MRI) and is primarily pronounced in the hippocampus, the entorhinal cortex and the amygdala (Bottino et al., 2002). The middle frontal gyri are affected but to a lesser extent than the temporal cortex, whereas the occipital and sensorimotor cortices are relatively spared in AD patients (Thompson et al., 2001).

\subsubsection{Synaptic loss}

$\mathrm{AD}$ patients were shown to have synaptic loss in different layers of the temporal and frontal cortex (Davies et al., 1987). It has been shown recently that patients with mild AD had $55 \%$ synaptic loss in the CA1 while patients with MCI exhibited $18 \%$ synaptic loss in comparison to control cases (Scheff et al., 2007). The degree of synaptic loss has been suggested to correlate with the duration of the dementia (Blennow et al., 1996). Since synaptic loss was found to parallel the cognitive state of the patients (Scheff et al., 2007), there was a significant correlation between synaptic count and the performance in the mini mental state examination (MMSE) in AD patients (DeKosky and Scheff, 1990). Besides, it has been suggested to be the major correlate to the cognitive decline in comparison to NFT and neuron loss (Terry et al., 1991; Scheff et al., 2007). This evidence is supported by the common knowledge that the synapses are the major sites for long term potentiation (LTP) and plasticity in the neuron (Scheff et al., 2007). 


\subsubsection{Axonopathy}

Many neurodegenerative diseases, such as Parkinson's disease, amyotrophic lateral sclerosis and AD, exhibit prominent axonal transport deficits which manifest as axonal swellings (De Vos et al., 2008). In early-AD patients, axonal abnormalities have been detected in many brain regions, such as the hippocampus (Probst et al., 1983), the cerebral cortex and subcortical regions such as the substantia nigra (McNeill et al., 1984) and the nucleus basalis of Meynert (Stokin et al., 2005), suggesting that axonal transport impairment plays a role

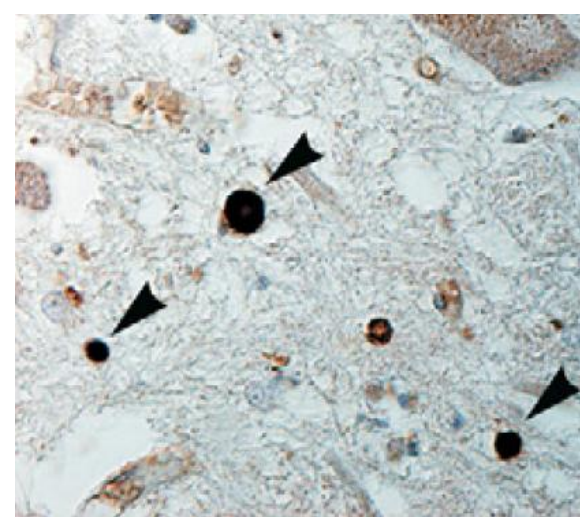

Fig 4. Axonopathy in AD. APPpositive axonal spheroids (arrow heads) in the spinal cord of an $\mathrm{AD}$ patient (Wirths et al., 2007).

during the pathology of the disease. Moreover, in vivo MRI studies illustrated that axonal transport is also altered in rodent models of AD (Smith et al., 2007).

Axonal swellings in AD have been shown to accumulate axonal cargoes or proteins like tau (Kowall and Kosik, 1987), phosphorylated tau, choline acetyltransferase, APP (Wirths et al., 2007), ubiquitin (Cras et al., 1991) and cellular organelles (Stokin et al., 2005). It is noteworthy to mention two types of axonal swellings present in the AD brain. The first type is dystrophic neurites seen in the vicinity of plaques that are formed in response to plaque deposition (Terry et al., 1964; Cras et al., 1991). Unlike plaque-dependent dystrophic neurites, plaque-independent axonal spheroids precede aberrant amyloid deposition or tau hyperphosphorylation and might rather represent a precursor for plaque deposition (Stokin et al., 2005) (Fig. 4).

\subsection{Diagnosis and treatment}

\subsubsection{Diagnosis}

Definite diagnosis of AD can only be achieved by examining postmortem tissue for amyloid plaques and NFTs. Nevertheless, the medical history of the patient obtained from a third informant is a basic practice in the diagnosis. Neurological and physical examinations serve as important parts of the differential diagnosis of the dementia. Also, cognitive assessment is an essential requirement especially at the early stages because it can detect 
memory impairments (Waldemar et al., 2007). This involves neuropsychological tests covering different cognitive entities, such as MMSE (Folstein et al., 1975). Blood tests are necessary to identify secondary causes of dementia and coexisting disorders that are common in elderly people. Neuroimaging (MRI and CT), is mainly used to exclude alternative causes of dementia such as brain tumors and subdural haematoma (Waldemar et al., 2007). Measurements of atrophy in certain regions of the temporal lobe using MRI hold promise to differentiate AD from control cases (Killiany et al., 1993). Hypometabolism in the temporal, parietal, and posterior cingulate cortex, identified by ${ }^{18} \mathrm{~F}$-fluorodeoxyglucose-positron emission tomography (PET), could be used to differentiate patients with AD from cognitively normal elderly people with high sensitivity and with a relatively good specificity to other dementias (Silverman et al., 2001; Herholz et al., 2002). In addition, cerebral spinal fluid (CSF) biomarkers, such as reduced $\mathrm{A} \beta_{\mathrm{x}-42}$ and increased tau and phosphorylated tau, have been investigated as valuable diagnostic biomarkers for AD (Mattsson et al., 2009).

\subsubsection{Treatment}

Despite all the vast efforts to investigate therapeutics for $\mathrm{AD}$, there is presently no cure. The available AD treatments target the symptoms and do not address the underlying molecular mechanism of the disease. Four drug-treatments, which target neurotransmitter disturbances in AD, are currently approved. These include the drug memantine and three acetylcholinesterase inhibitors. In addition, some antipsychotic drugs are moderately effective in alleviating some AD symptoms, such as aggression and psychosis (Ballard and Waite, 2006).

Memantine is a non-competitive N-methyl D-aspartate (NMDA)-receptor antagonist. It works by protecting neurons from glutamate-mediated excitotoxicity that is increased in AD without preventing the physiological NMDA-receptor activation needed for cognitive functioning. Memantine shows modest effects in moderate to severe AD cases (Areosa et al., 2005).

The cholinergic hypothesis postulates that the activity of cholinergic neurons is disturbed in AD, leading to cognitive and memory impairment (Terry and Buccafusco, 2003). With this notion in mind, three drugs, donepezil, rivastigmine and galantamine, are used to 
increase the availability of acetylcholine by inhibiting acetylcholinesterase, the enzyme that degrades acetylcholine in the synaptic cleft. Rather than changing the natural course of AD, they only temporarily mitigate some of the symptoms (Bullock and Dengiz, 2005; Bullock et al., 2005; Winblad et al., 2006).

\subsection{The amyloid precursor protein}

The similarities in the brain pathology between $\mathrm{AD}$ and Down syndrome (DS) raised the question of whether a common molecular basis underlies the pathology in both situations. The localization of the defective gene in familial AD (FAD) to the DS region of chromosome 21 provided an explanation for the AD phenotype seen in DS (St George-Hyslop et al., 1987). The defective gene was shortly isolated and described to be a single transmembrane glycoprotein, the amyloid precursor protein (APP) (Kang et al., 1987; Tanzi et al., 1987).

APP belongs to a gene family that includes amyloid precursor like protein-1 (APLP-1) and APLP-2 which are type-1 transmembrane proteins and share significant sequence homology in their ectodomains; the A $\beta$ sequence, however, is unique to APP (Bayer et al., 1999). The APP gene encodes three major isoforms, APP695, APP751 and APP770, resulting from alternative splicing (Kang et al., 1987; Kitaguchi et al., 1988; Tanzi et al., 1988). APP770 and APP751 are expressed widely in non-neuronal cells throughout the body but also expressed in neurons. APP695, which is expressed predominantly in neurons (Sola et al., 1993), is the most abundant form in the brain. In both human and rodents, APP expression shows wide distribution in the brain with the highest expression in the cortex, hippocampus and cerebellum. APP was reported in many other brain regions such as the basal ganglia (nucleus basalis of Meynert) and the substantia nigra (Sola et al., 1993). In neurons, APP is expressed in neuronal perikarya, transported by fast anterograde axonal transport and found in the nerve terminals, dendrites and synapses (Koo et al., 1990).

Although the pathological role of APP has been extensively studied since its discovery, the physiological role is still undetermined. APP has been suggested to be involved in cell adhesion, neurite growth, synaptogensis, neuronal transport of proteins, transmembrane signal transduction and calcium metabolism (Zheng and Koo, 2011). However, studies have 
shown that APP null mice are viable and fertile despite their reduced body weight, locomotor activity, cognitive impairment and reduced LTP in addition to gliosis (Dawson et al., 1999).

After its synthesis in the endoplasmic reticulum (ER), APP is subject to posttranslational modifications such as glycosylation, sulfation, and phosphorylation during its transit through the intracellular protein secretory pathway (Selkoe, 2001). APP isoforms exist such as the immature form (imAPP) that is localized to the ER and cis-Golgi and not subject to cleavage by secretases. imAPP is then trafficked though the Golgi to yield the mature APP (mAPP) (Tomita et al., 1998). mAPP in neurons is phosphorylated after transit through the Golgi apparatus, just preceding APP targeting out of neuronal perikarya into the axons (Suzuki et al., 1997). Phosphorylation of APP is suggested to regulate its subcellular trafficking, production of $\mathrm{A} \beta$, cell signaling and protect it against degradation by caspases (Lee et al., 2003; Suzuki and Nakaya, 2008).

\subsubsection{Non-amyloidogenic pathway}

There are two major alternative pathways for APP processing that differ in the enzymes involved as well as in the resulting peptides. In the non-amyloidogenic pathway, alpha secretase ( $\alpha$-secretase) cleaves APP twelve amino acids from the transmembrane domain at the N-terminal. This cleavage occurs in the $\mathrm{A} \beta$ domain and demolishes the production of $\mathrm{A} \beta$ (Sisodia et al., 1990). It occurs primarily at the plasma membrane resulting in shedding of the soluble ectodomain fragment $(\mathrm{sAPP} \alpha)$ into the lumen/extracellular space and retention of an 83-residue C-terminal fragment (CTF $\alpha)$ in the membrane (Sisodia, 1992). $\alpha$-Secretase is a single transmembrane type- 1 protein which was found to be zinc-dependent

(Roberts et al., 1994) (Fig. 5). Enzymes belonging to ADAM (a disintegrin and metalloproteinase) family such as ADAM 9, ADAM 10 and ADAM 17 have been suggested to have $\alpha$-secretase activity (Buxbaum et al., 1998; Koike et al., 1999; Lammich et al., 1999). The remaining CTF $\alpha$ is further processed by gamma secretase ( $\gamma$-secretase) to release the short p3 fragment and the APP intracellular domain (AICD) (Zhang et al., 2011). 


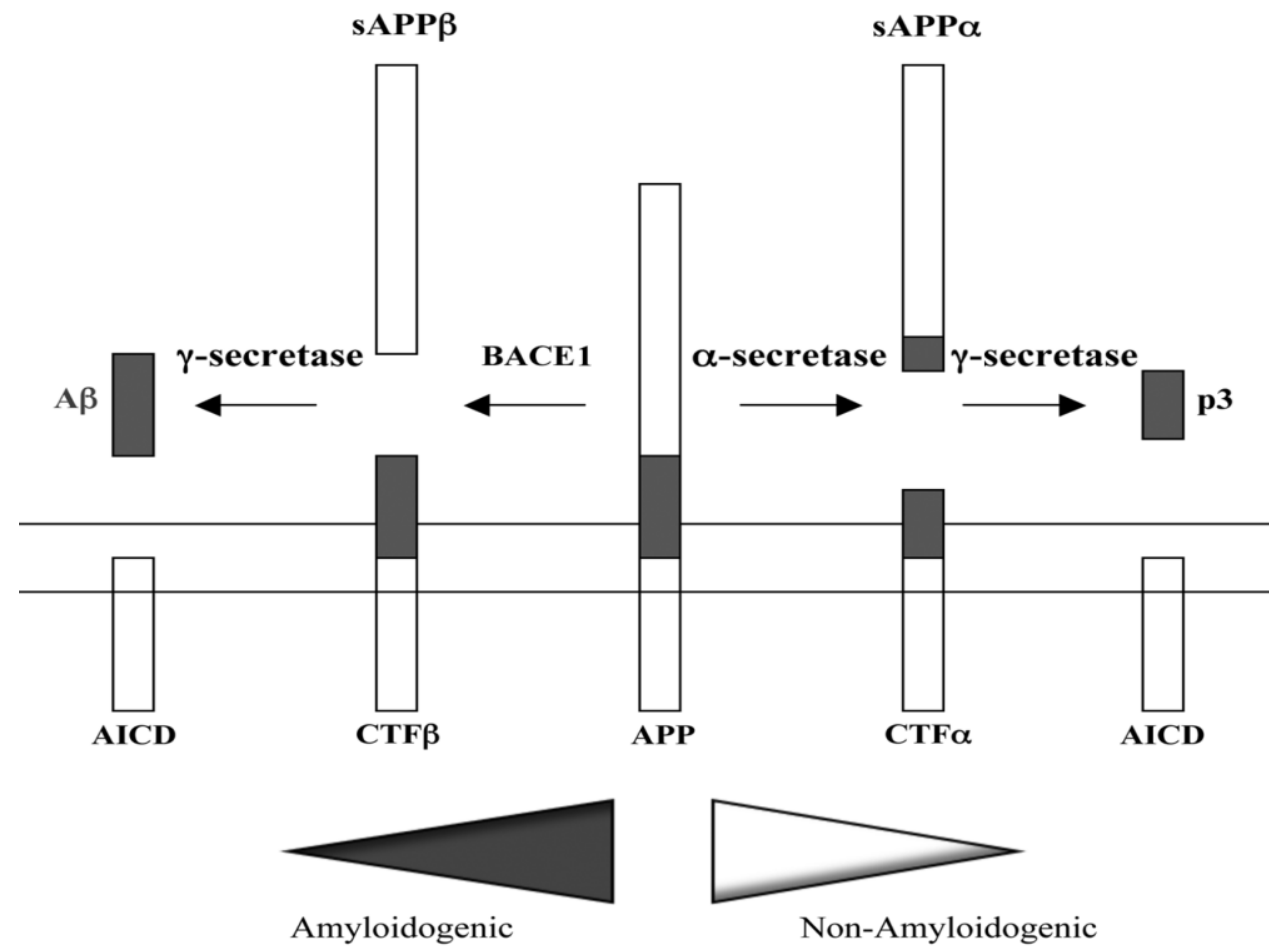

Fig. 5. APP processing. Schematic representation showing the non-amyloidogenic processing via $\alpha$-secretase and $\gamma$-secretase to produce p3 (right) and the amyloidogenic precessing via BACE1 ( $\beta$-secretase) and $\gamma$-secretase to produce amyloid plaque-forming A $\beta$ (left). Taken from (Tang et al., 2009)

\subsubsection{Amyloidogenic pathway}

The amyloidogenic pathway involves the cleavage of APP by beta secretase $(\beta$ secretase), which cuts 16 amino acids upstream from the $\alpha$-secretase cleavage site to generate a soluble amino terminal APP derivative ( $\mathrm{SAPP} \beta)$, and a membrane inserted C-terminal fragment that is 99 amino acids long (CTF $\beta)$. After the initial $\beta$-secretase cut, $\gamma$-secretase cleaves the CTF $\beta$ at different intramembranous sites to yield $A \beta$ peptides of varying lengths (39 - 43 amino acids) consisting mostly of $A \beta$ that is 40 amino acids long $\left(A \beta_{x-40}\right)$ and a smaller amount of $A \beta$ that is 42 amino acids long $\left(A \beta_{x-42}\right)$ in addition to the AICD (Citron et al., 1995; Haas et al., 1995; Selkoe, 2001; Tang, 2009).

The beta site APP-cleaving enzyme (BACE) was identified as $\beta$-secretase concomitantly by several groups (Hussain et al., 1999; Sinha et al., 1999; Vassar et al., 1999). BACE1 (or Asp2) is a membrane-bound aspartyl protease with a characteristic type-1 transmembrane domain near the $\mathrm{C}$-terminus that cleaves $\mathrm{A} \beta$ at known positions; the aspartate at position 1 or the glutamate at position 11 (Sinha et al., 1999; Vassar et al., 1999). BACE1 is 
highly expressed in the brain, especially in the hippocampus, the cortex and the cerebellum, and is considered the major $\beta$-secretase for the generation of $A \beta$ peptides by neurons (Vassar et al., 1999; Cai et al., 2001). At a subcellular level, BACE1 is predominantly localized within endosomes, with lower levels in the trans-Golgi network (TGN) and the cell membrane; it is absent from lysosomes and the ER (Huse et al., 2002). Very little BACE1-mediated APP cleavage occurs at the plasma membrane because it is recycled very quickly, precluding substrate cleavage. Instead APP is cleaved by BACE1 primarily in endocytic vesicles (Chow et al., 2011). In addition to BACE1, BACE2 and cathepsin B have been suggested to exert $\beta$ cleavage of APP, but their physiological contribution to APP cleavage is still debatable (Zhang et al., 2011).

$\gamma$-Secretase consists of at least of four components: presenilin (PS; PS1 or PS2), nicastrin, anterior pharynx-defective-1 (APH-1) and presenilin enhancer-2 (PEN-2) (Kimberly et al., 2003) (Fig. 6). This complex is suggested to reside primarily in the ER, Golgi/TGN, endocytic and intermediate compartments (Cupers et al., 2001). Besides cleaving APP CTFs, $\gamma$-secretase cleaves other substrates such as notch, cadherin, tyrosinase, ErbB4 and CD44 (Wolfe, 2010). Cleavage of APP CTFs was suggested to occur in TGN and in the early endosomes (Xu et al., 1997; Greenfield et al., 1999).

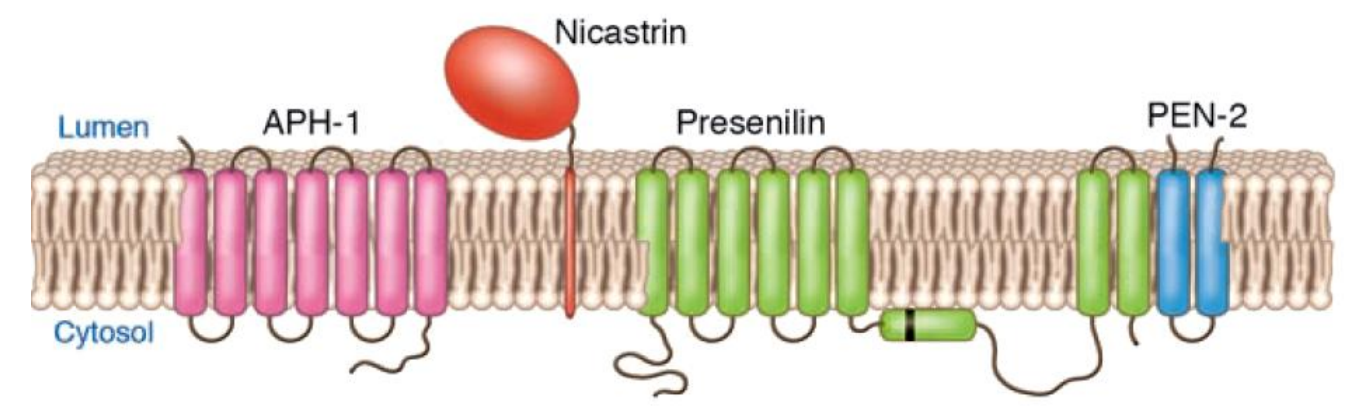

Fig 6. Topology of $\gamma$-secretase complex. $\gamma$-Secretase consists of four components which are presenilin, nicastrin, PEN-2 and APH-1. The black bar represents the cleavage site for processing of PS1 into the amino and carboxyterminal fragments that self associate and form the active enzyme. Taken from (Gandy, 2005).

\subsubsection{A $\beta$ aggregation}

In vitro and in vivo analysis of amyloid deposits in $\mathrm{AD}$ revealed various $\mathrm{N}$ - and $\mathrm{C}$ terminal variants (Masters et al., 1985b; Suzuki et al., 1994a). Although the predominant A $\beta$ 
species in congophilic amyloid angiopathy (CAA) was suggested to be $A \beta_{\mathrm{x}-40}$ (Glenner and Wong, 1984), the major C-terminal $A \beta$ isoform in plaques was found to be $A \beta_{x-42}$ (Prelli et al., 1988; Miller et al., 1993). The increased C-terminal length of A $\beta$ in AD appears to cause the enhanced aggregation, early deposition and promote the toxicity of $\mathrm{A} \beta$ (Barrow and Zagorski, 1991; Iwatsubo et al., 1994; Pike et al., 1995).

The properties of $\mathrm{A} \beta$ peptides enable it to self-associate into different aggregation states ranging from monomers, soluble oligomers and insoluble fibrils. A $\beta$ can produce oligomers ranging from dimers to high molecular weight structures (larger than $100 \mathrm{kDa}$ ), although, the majority of $\mathrm{A} \beta$ occurs in lower molecular weight ranges, from dimers to octamers. Soluble A $\beta$ oligomers are low molecular weight, non-fibrillar, soluble structures which remain stable in aqueous solution even after high speed centrifugation (Walsh and Selkoe, 2007). A $\beta$ monomers are suggested to be in equilibrium with different oligomeric isoforms. When a certain threshold of monomers is reached, polymerization into paranuclei is favored. These paranuclei self associate to form protofibrils which are beaded chains of $5 \mathrm{~nm}$ in diameter and less than $150 \mathrm{~nm}$ in length. Maturation of these protofibrils yields either the classical fibrils (around $10 \mathrm{~nm}$ in diameter and often more than $1 \mu \mathrm{m}$ in length) comprising amyloid plaques, or alternatively, they can depolymerize to lower-order species (Walsh and Selkoe, 2007; Selkoe, 2008; Roychaudhuri et al., 2009) (Fig. 7).

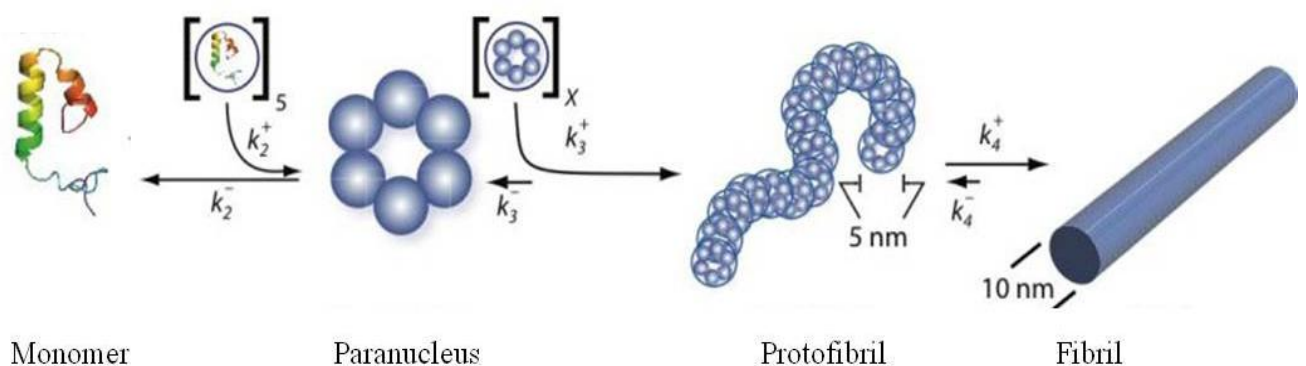

Fig 7. A $\beta$ aggregation. $A \beta$ is a natively disordered protein, existing in the monomeric state as an equilibrium mixture of many conformers. Formation of a partially folded monomer is required to produce a nucleus for fibril elongation, a paranucleus (in this case, containing six monomers). Paranuclei self associate readily (to form protofibrils which comprise a significant but finite number of paranuclei. Maturation of protofibrils yields the classical amyloid-fibrils. Adapted from (Roychaudhuri et al., 2009). 


\subsection{The amyloid hypothesis}

For more than two decades, the amyloid hypothesis has been the cardinal hypothesis describing $\mathrm{AD}$ etiology. The amyloid hypothesis considers $\mathrm{A} \beta$ deposition to be the causative event of AD pathology; neurofibrillary tangles, cell loss, vascular damage and dementia occur as a consequence of this event (Hardy and Allsop, 1991). The evolution of this hypothesis was supported by many observations. One of the strongest arguments for the amyloid hypothesis came from genetic studies which brought to light many FAD cases that resulted from inherited APP or PS1 mutations leading to increased deposition of A $\beta$ in plaques (Bertram et al., 2010). In addition, triplication of the APP gene in DS patients leads to AD pathology in these patients (St George-Hyslop et al., 1987). Moreover, transgenic models of AD harboring APP mutations develop age-dependent pathology similar to that seen in AD (Philipson et al., 2010). Also, the fourth allelic variant ( $\varepsilon 4$ ) of the apolipoprotein (APOE) polymorphism, which represents the most important genetic risk factor for the sporadic form of $\mathrm{AD}$, is associated with $A \beta$ accumulation and reduced clearance (Castellano et al., 2011). On the other hand, mutations in tau lead to taupathies and not $A D$, thereby suggesting that NFTs occur subsequent to $A \beta$ aggregation.

One of the major flaws in the amyloid hypothesis is the weak correlation between the severity of the dementia and the density of amyloid plaques (Snowdon, 2003). Reciprocally, cognitive impairments and pathological changes in many AD mouse models occur before the first signs of the plaque deposition (Klein et al., 2001; Walsh and Selkoe, 2007). Johansson et al. (2007) studied the effect of the polyunsaturated fatty acid docosahexaenoic acid on $A \beta_{x-42}$ oligomerization and fibrillization in vitro and showed that it stabilized soluble $A \beta_{x-42}$ protofibrils, thereby hindering their conversion to insoluble fibrils and hence maintaining $A \beta$ induced toxicity over time. On the other hand, $A \beta_{x-42}$ devoid from docosahexaenoic acid demonstrated reduced toxicity, as A $\beta$ peptides formed fibrils (Johansson et al., 2007). 


\subsubsection{The intraneuronal amyloid hypothesis}

The neuronal

The Modified $\beta$-Amyloid Cascade

origin of the extracellular plaques is one of the accepted facts in the field of $\mathrm{AD}$. In vitro studies have elucidated the presence of intraneuronal $\mathrm{A} \beta$ in cultured human neurons (Wertkin et al., 1993; Turner et al., 1996). The first observations about the presence of $A \beta$ in neurons, and that it precedes plaque deposition in $\mathrm{AD}$ cases, date back more than 20

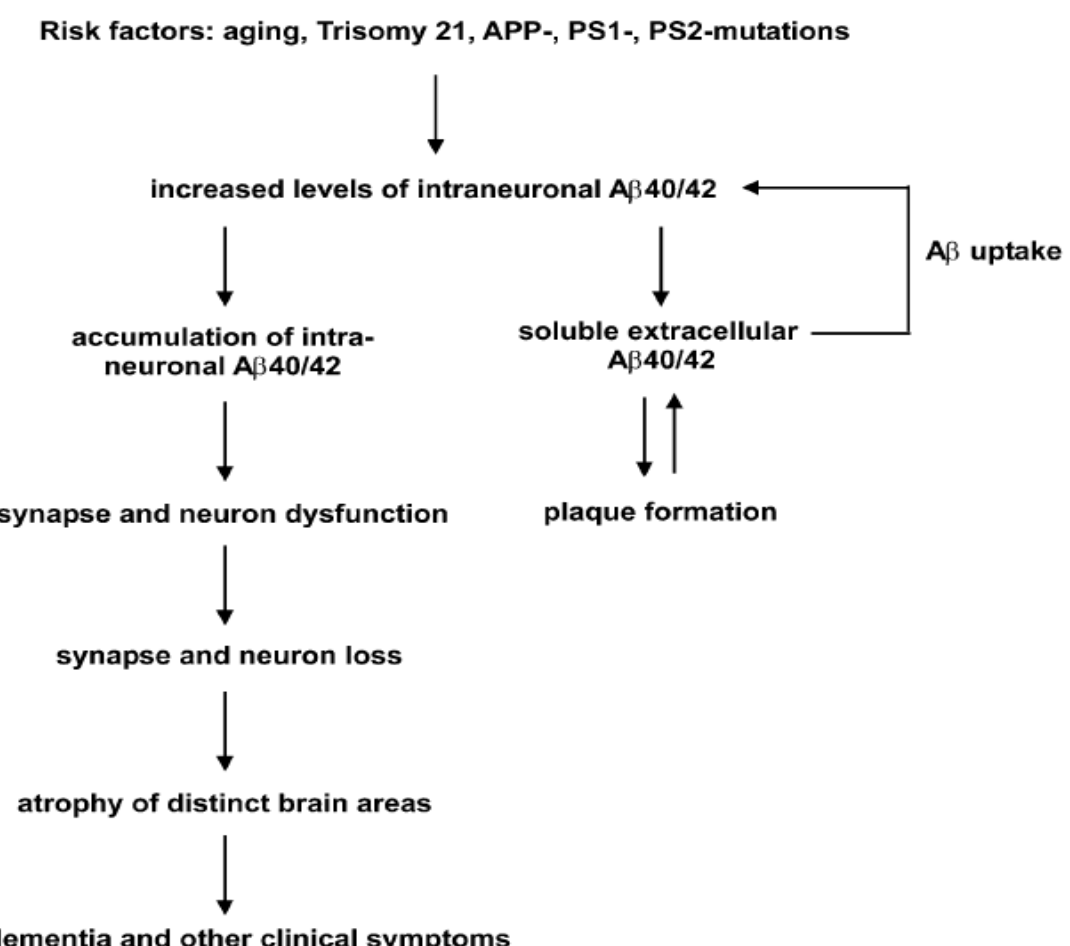

Fig 8. Representation of the intraneuronal Aß hypothesis. (Wirths et al., 2004).

years (Masters et al., 1985a; Grundke-Iqbal et al., 1989). Moreover, a study by Gouras et al. showed that intraneuronal $\mathrm{A} \beta_{\mathrm{x}-42}$ accumulates in $\mathrm{AD}$ vulnerable regions and that this deposition precedes NFTs and extracellular plaques (Gouras et al., 2000). A similar finding was made in young DS patients lacking plaque deposition. This observation led to the suggestion that intracellular $\mathrm{A} \beta$ deposition precedes extracellular deposition and pathological consequences (Gyure et al., 2001). An increasing amount of data regarding intraneuronal A $\beta$ in $\mathrm{AD}$ has accumulated in the past years showing the correlation between intraneuronal $\mathrm{A} \beta$, neuron loss and cognitive impairments in several mouse models of AD (Wirths and Bayer, 2010) (Fig. 8).

Converging evidence points toward two different origins of intraneuronal $A \beta$ : intracellular processing of APP and reuptake of A $\beta$ from the extracellular space. Extensive studies support the role of intracellular APP processing in the accumulation of intraneuronal 
$A \beta$. Several reports have shown that $A \beta_{x-42}$ is generated in the ER/intermediate compartments and TGN while $A \beta_{x-40}$ is generated exclusively in the TGN (Cook et al., 1997; Hartmann et al., 1997; Greenfield et al., 1999). It has been suggested that clathrin mediated endocytosis of cell surface APP leads to the production of A $\beta$ via the endocytic pathway (Koo and Squazzo, 1994).

In addition to intracellular production of $A \beta$, extracellular reuptake of $A \beta$ represents another source of intraneuronal $A \beta$. Several pathways have been proposed for the internalization of $\mathrm{A} \beta$ like APOE receptors, endocytosis, nicotinic acetyl choline receptors, integrins, NMDA receptors and the receptor for advanced glycation end products (Mohamed and Posse de Chaves, 2011).

Accumulation of $A \beta$ in lysosomes leads to the loss of lysosomal impermeability and leakage of lysosomes' content, such as cathepsins and proteases, resulting in necrosis and apoptosis (Ditaranto et al., 2001; D'Andrea et al., 2002). Also, accumulation of $A \beta$ in multivesicular bodies (MVBs) might hinder their function in retrograde transport and degradation. This is particularly important since MVBs represent a good medium for A $\beta$ aggregation due to their abundant membranes and acidic environment (Takahashi et al., 2002). In vitro and in vivo studies have shown that accumulation of intraneuronal $\mathrm{A} \beta$ impairs protease activity and leads to tau accumulation (Tseng et al., 2008). Moreover, the localization of $A \beta$ to mitochondria in APP models correlated with a reduced rate of oxygen consumption, increased hydrogen peroxide and decreased respiratory chain enzymes activity (Caspersen et al., 2005; Manczak et al., 2006). Studies from AD mouse models and AD patients showed a correlation between intraneuronal $A \beta$ and apoptotic markers (Chui et al., 2001).

All together, these findings from AD patients, DS patients, mouse models and in vitro studies led to a twist in the amyloid hypothesis which identifies intraneuronal $A \beta$ as a key player in AD pathology and may solve the flaws in the original amyloid hypothesis (Wirths et al., 2004).

\subsection{Genetics}

$\mathrm{AD}$ is a multifactorial disease that is influenced by many environmental and inherited factors, with aging as the major risk factor for the disease. After the age of 65 , the risk of 
developing AD doubles every 5 years; thus, the global incidence of $\mathrm{AD}$ raises as the average age of the worldwide population increases (Ferri et al., 2005). Besides aging, low educational and occupational attainment, low mental ability in early life and reduced mental and physical activity during late life have been suggested to increase the risk of AD (Whalley et al., 2000; Scarmeas et al., 2001; Lindsay et al., 2002). Other factors may include traumatic head injuries, depressive mood and excessive alcohol consumption (Mayeux, 2003). Moreover, a particularly high risk of developing $\mathrm{AD}$ has been shown for smokers and patients with hypertension, diabetes or hyperlipidemia (Lange-Asschenfeldt and Kojda, 2008).

The vast majority of $\mathrm{AD}$ cases are sporadic, while 5 to $10 \%$ are familial $\mathrm{AD}$ cases (FAD) caused by inherited mutations usually leading to early onset AD. Early onset AD includes patients who range from 16 to 65 years of age; whereas late onset AD includes patients older than 60 years. Genetic studies of FAD revealed three main gene mutations in the APP, PS1 and PS2 genes. These mutations account for only 30-50\% of the cases with autosomal dominant AD (Bekris et al., 2010).

The first mutation detected in the APP was discovered in British kindred and thus called the London mutation (Goate et al., 1991). Currently, more than 32 APP missense mutations have been identified in 89 families (Department of molecular genetic, University of Antwerp, 2011). All APP mutations identified so far lie in or near the A $\beta$ sequence. The majority of APP mutations are in the vicinity of protease cleavage sites, exerting their pathogenic effect by influencing APP cleavage and altering the $A \beta_{x-42} / A \beta_{x-40}$ ratio. However, the Swedish mutation at the $\beta$-secretase cleavage site increases the overall A $\beta$ levels (Mullan, 1991; Citron et al., 1992). Mutations near the $\gamma$-secretase cleavage site affect the C-terminal cleavage position. The London mutation (APP717) near the $\gamma$-secretase cleavage sites leads to an increase in the $A \beta_{x-42}$ over $A \beta_{x-40}$ ratio without affecting the overall $A \beta$ production (Suzuki et al., 1994b). The same holds true for the Florida mutation (APP716) mutation (Eckman et al., 1997). Mutations at the $\alpha$-secretase cleavage site, such as the Flemish mutation (APP692), interfere with $\alpha$-secretase cleavage, giving more substrate for the $\beta$-secretase (Cras et al., 1998). On the other hand, mutations within the $A \beta$ sequence may alter the physiochemical properties of $\mathrm{A} \beta$, leading to enhanced aggregation and increased protofibril/fibril formation, 
an observation seen in the Dutch and the Arctic mutations (Nilsberth et al., 2001; Brouwers et al., 2008) (Fig. 9).

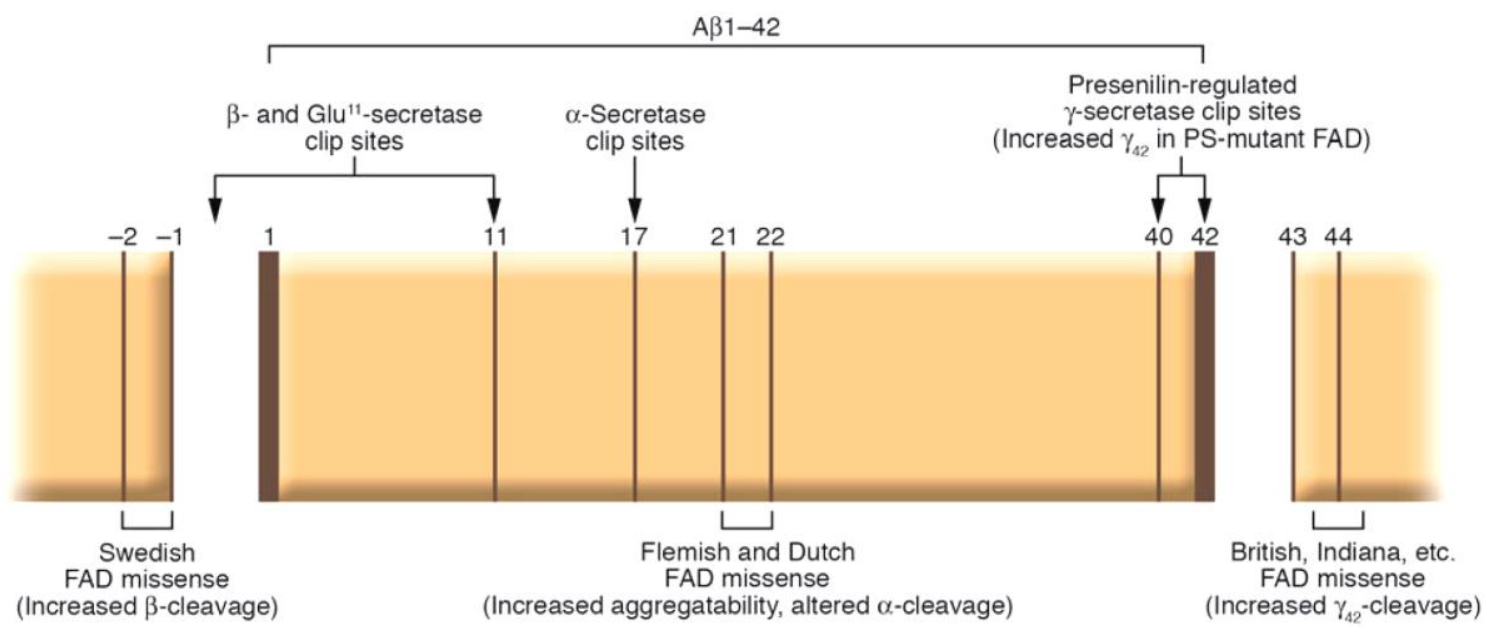

Fig 9. Some of the AD missense mutations. The Swedish mutation increases $\beta$-secretase cleavage, while the Flemish mutation partly disfavors $\alpha$-cleavage of APP. The Arctic, Dutch and Iowa mutations mainly increase aggregation. The London-type APP mutations increase $A \beta_{x-42}$ or the $A \beta_{x-42} / A \beta_{x-40}$ ratio by altering the $\gamma$ secretase cleavage site. Taken from (Gandy, 2005).

Linkage studies revealed the presence of another locus on chromosome 14 related to FAD which corresponds to the PS1 gene. Mutations in PS1 are the most common causes of FAD, accounting for $18-50 \%$ of cases of FAD. To date, 182 PS1 mutations have been identified in 402 families (Department of molecular genetic, University of Antwerp, 2011). Besides being the most common FAD mutation, it accounts for the most aggressive and early form of the disease, characterized by an onset of 30 years of age (Bekris et al., 2010). In addition to mutations in PS1, mutations in PS2 gene on chromosome 1 were identified; however, they represent a rare cause of FAD. So far, 13 mutations in the PS2 have been described in 22 families (Department of molecular genetic, University of Antwerp, 2011). PS1 and PS2 mutations are known to cause an increase in $\mathrm{A} \beta_{\mathrm{x}-42} / \mathrm{A} \beta_{\mathrm{x}-40}$ ratios either by increasing $\mathrm{A} \beta_{\mathrm{x}-42}$ or by decreasing $\mathrm{A} \beta_{\mathrm{x}-40}$ levels (Kumar-Singh et al., 2006).

Although genetic mutations account for a minority of AD cases, several genetic polymorphisms have been suggested to be associated with sporadic AD such as the fourth allelic variant $(\varepsilon 4)$ of APOE, which represents the strongest genetic risk factor. The presence of the APOE $\varepsilon 4$ allele increases the risk of the disease by 3 -fold in heterozygous carriers and by 15 -fold in homozygous carriers (Farrer et al., 1997). Other susceptibility loci that might 
contribute, but to a lesser extent, to the risk of developing $\mathrm{AD}$ include clusterin (CLU), complement component $(3 \mathrm{~b} / 4 \mathrm{~b})$ receptor 1 (CR1) and phosphatidylinositol-binding clathrin assembly protein (PICALM) (Sleegers et al., 2010).

\subsection{Mouse models of Alzheimer's disease}

Transgenic mouse models of AD have been developed based on the amyloid hypothesis and advanced from the contentious discovery of mutations in FAD. AD mouse models, like other disease models, contribute widely to the understanding of the pathophysiology of the disease. Furthermore, they have been invaluable tools in the preclinical testing of potential therapeutics such as immunotherapies. Whereas invertebrate models such as Drosophila melanogaster and Caenorhabditis elegans are available for various diseases, including $\mathrm{AD}$, and might offer certain advantage over mouse models, they are evolutionarily distant from humans. Mice, on the other hand, are the most commonly used vertebrates for transgenic modeling due to their relatively short life span, low costs and easy handling. Moreover, the use of mice has been facilitated by the availability of advanced techniques and knowledge of genetic modification. Although some transgenic rats have been described recently (Philipson et al., 2010), mice are almost the exclusive transgenic mammals used to model AD.

The discovery of the FAD mutations in APP provided the opportunity to study AD through the generation of $\mathrm{AD}$ models carrying these mutations. This was started with the generation of a mouse model that overexpresses the Indiana mutation, V717F, under the platelet-derived growth factor. This drives the expression of the transgene in the CNS and the periphery, leading to an 18-fold increase in APP levels. As a consequence, the mice exhibit an age-dependent increase in plaque deposition starting from 6 months of age, in addition to dystrophic neurites, astrocytosis, microgliosis and synaptic loss (Games et al., 1995; Rockenstein et al., 1995). These mice show deficits in object recognition memory which were found to be related to the number of amyloid deposits in specific brain regions, whereas deficits in spatial reference and working memory were related to the changes in synaptic density and hippocampal atrophy (Dodart et al., 2000). The Tg2576 mouse is one of the most widely studied models, expressing the Swedish mutation under the control of the hamster 
prion protein promoter. It develops plaque pathology around 9-12 months of age together with astrocytosis, microgliosis, dystrophic neurites and impairments in different forms of memory such as spatial and working memory (Hsiao et al., 1996). Moreover, other mouse models with multiple APP mutations have been generated to drive early AD pathology such as the TgCRND8 mouse model (Chishti et al., 2001).

Many PS models have been generated, either using similar promoters to the APP models, such as the PS1 M146L model (Duff et al., 1996), or as targeted genes, such as the PS1M146VKI model (Guo et al., 1999). In general, PS1 models exhibit increased $A \beta_{x-42}$ levels, but they do not develop an overt deposition of plaques or other AD hallmarks, unless they are crossed to APP transgenic mice (Holcomb et al., 1998). The lack of plaque deposition in the PS1 models might be due to the low $\mathrm{A} \beta_{\mathrm{x}-42}$ levels in comparison to APP models. It might also be caused by differences in the solubility and aggregation properties between mouse and human A $\beta$ (Jankowsky et al., 2007).

Although AD mouse models represent a wide spectrum of AD pathology, they are hampered by the lack of some cardinal features of AD, such as neuron loss and NFTs. Among APP models, only APP23, with the Swedish mutation under the Thy-1 promoter, harbors neuron loss in the CA1 region at 14-18 months of age (Calhoun et al., 1998). More widespread neuron loss has been reported in mouse models which coexpress APP and PS1 mutations, such as the APP/PS1KI (Casas et al., 2004), APP/PS1 (Schmitz et al., 2004) and 5XFAD mouse models (Oakley et al., 2006). Hence, neuron loss seems to be detected in mice where a combination of multiple mutations is expressed.

Another weakness is the lack of NFTs in AD models. In order to overcome this, FAD and tau mutations from frontotemporal dementias were combined in mice like the tau/APP mice resulting from the crossing of $\mathrm{Tg} 2576$ and P301 mice (Lewis et al., 2001). A triple transgenic model, $3 \times \mathrm{Tg}$, was generated by coinjecting two transgenes containing APPSwedish and P301L mutations into PS1M146V knock-in mice (Oddo et al., 2003). Both models produce NFT-like pathology in addition to plaque deposition. 


\subsection{The 5XFAD mouse model}

The 5XFAD model is a double transgenic APP/PS1 mouse model that co-expresses five AD mutations leading to accelerated plaque formation and increased $\mathrm{A} \beta_{\mathrm{x}-42}$ production. The model was generated by introducing APP Swedish, Florida and London mutations, and PS1 M146L and L286V mutations into

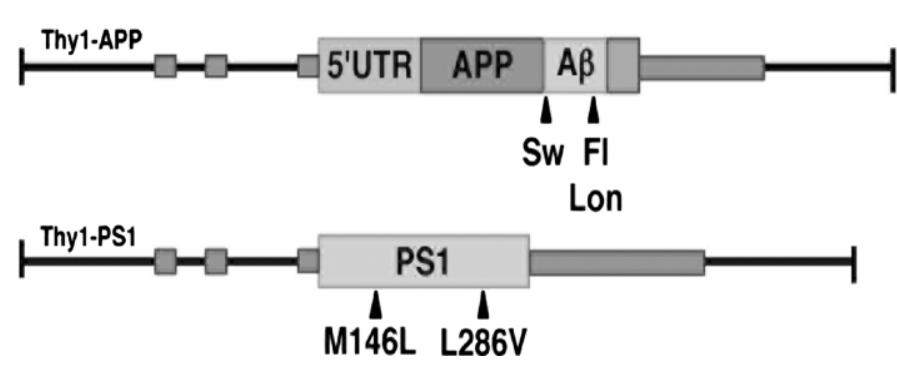

Fig 10. Schematic diagram of the 5XFAD, APP and PS1, transgenes. FAD mutations in Thy1-APP and Thy1-PS1 transgenes are indicated by arrowheads. Sw, Swedish mutation; Lon, London mutation; Fl, Florida mutation. Small gray rectangles represent coding regions of APP and PS1. Taken from (Oakley et al., 2006). APP (695) and PS1 cDNAs via sitedirected mutagenesis. They were later sub-cloned into mouse Thy-1 transgenic cassette. The originally described 5XFAD mice were maintained in a hemizygous state on a C57/BL6SJL background. The mice showed stable transmission and expression of both APP and PS1 transgenes over generations, demonstrating that the 5XFAD mice breed as a single transgenic model (Oakley et al., 2006).

The presence of Swedish mutation results in higher levels of the total $A \beta$, whereas Florida, London, M146L and L286V mutations increase the production of $A \beta_{x-42}$ specifically (Fig. 10). Therefore, $5 X F A D$ mice show a dramatic increase in $A \beta_{x-42}$ in comparison to $A \beta_{x-40}$ which is proportional to the APP expression. This is reflected by an early onset of plaque deposition (around 2 months of age) that spreads to cover most of the brain paralleled with astrocytosis and microgliosis (Oakley et al., 2006). Interestingly, 5XFAD mice exhibit a gender-based effect. This was illustrated when mice showed a gender-specific response to stress in which female mice exhibited a significant increase in hippocampal plaque deposition that was not seen in male mice (Devi et al., 2010).

In addition to plaque pathology and gliosis, the 5XFAD model was reported to develop age-dependent synaptic degeneration indicated by the reduction of synaptic markers (Oakley et al., 2006). Intraneuronal BACE1 and $A \beta$ colocalize to swollen presynaptic terminals and fine axonal processes before plaque deposition. Later, these structures undergo a continuing 
process of sprouting/swelling and dystrophy, associated with the emergence and deposition of extracellular A $\beta$ (Zhang et al., 2009).

The 5XFAD mouse model represents an important model as it is one of the few currently available AD mouse models known to develop neuron loss. 5XFAD mice were reported to develop neuron loss by 9 months of age at the cortex and subiculum (Oakley et al., 2006). Genetic deletion of BACE in the 5XFAD/BACE knockout mice rescues the neuron loss in these mice in comparison to 5XFAD mice (Ohno et al., 2007). However, neuron loss in the 5XFAD mice was never appropriately quantified.

As a consequence of the extensive pathology seen in the 5XFAD mouse model, this model develops different behavioral impairments. 5XFAD mice were found to develop working memory impairments in the Y-maze as they reached an age of 4 to 5 months (Oakley et al., 2006). In addition, 5XFAD mice exhibit impairment in the contextual fear conditioning as early as 4 months of age, which correlates with hippocampal synaptic dysfunction (Kimura and Ohno, 2009). At 9 months of age, these mice are impaired in the conditioned taste aversion test, which can be rescued to the wild type performance by partially abolishing BACE (heterozygous knock-out) (Devi and Ohno, 2010).

All together, the variety of AD hallmarks presented by the 5XFAD model prompted its use recently to assess different potential therapeutic interventions, such immunization (Wirths et al., 2010b), somionine (Joyashiki et al., 2011), PKC activator (Hongpaisan et al., 2011), agonists of the peroxisome proliferator activated receptor gamma (PPARgamma) (Kalinin et al., 2009) and icariin (Urano and Tohda, 2010).However, as this model lacks NFTs formation, it represents an amyloid-predominant model of AD.

\subsection{The pyroglutamate-modified A $\beta$}

In order to unravel the pathogenic properties of $A \beta$, it was important to develop approaches to extract and study the biochemical nature of $\mathrm{A} \beta$. Limited extraction and sequencing methods rendered it impossible for a long time. Masters et al. reported the presence of ragged $\mathrm{N}$-termini of $\mathrm{A} \beta$ extracted from the plaque cores of $\mathrm{AD}$ and $\mathrm{DS}$ patients (Masters et al., 1985b). According to them, 64\% of the total A $\beta$ peptides started with phenylalanine at position 4. Soon after that, Selkoe and colleagues reported that they were not 
able to obtain $\mathrm{N}$-terminal sequences from plaques. Thus, they postulated that the $\mathrm{N}$-terminal of A $\beta$ might be blocked (Selkoe et al., 1986). In line with these suggestions, other teams did not succeed in obtaining interpretable N-terminal sequences from plaque cores isolated by other methods (Gorevic et al., 1986). Finally, this discrepancy was resolved by Mori and colleagues who described the presence of $A \beta$ peptides $(15-20 \%$ of the total $A \beta)$ bearing a pyroglutamate residue at the N-terminus. By using pyroglutamate amino peptidase, they were able to unravel the N-terminus, which is blocked by the lactam ring and thus resistant to any other peptidase previously used for Edman sequencing (Mori et al., 1992).

Since then, the interest in dissecting the temporal and spatial deposition of pyroglutamate-modified $\mathrm{A} \beta\left(\mathrm{A} \beta_{\mathrm{pE} 3-\mathrm{x}}\right)$ has increased. Many techniques and protocols were developed in order to increase the sensitivity to different forms of $A \beta$, especially the $A \beta_{\mathrm{pE} 3-\mathrm{x}}$ peptides (Naslund et al., 1996; Thompson et al., 1999). Equipped with a set of novel antibodies, Saido et al. showed by immunohistochemical and biochemical means that $A \beta_{\mathrm{pE} 3-\mathrm{x}}$ is present in equivalent or larger amounts than full-length $\mathrm{A} \beta$ in senile plaques. Based on the analysis of brain tissue from DS cases, the authors also suggested that $A \beta_{\mathrm{pE} 3-\mathrm{x}}$ precedes the deposition of unmodified $A \beta\left(A \beta_{1-x}\right)$ (Saido et al., 1995). However, a study on the sequential deposition of heterogenous forms of $A \beta$ in the brains of DS patients could not detect $A \beta_{\mathrm{pE}-\mathrm{x}}$ in young patients. Nevertheless, in agreement with Saido et al., A $\beta_{\mathrm{pE}-\mathrm{x}}$ always exceeded the deposition of $A \beta_{1-x}$ (Lemere et al., 1996). This was further confirmed by another study on water-soluble $\mathrm{A} \beta$ demonstrating the presence of $\mathrm{A} \beta_{\mathrm{pE} 3-42}$ in $\mathrm{AD}$ and $\mathrm{DS}$ as a dominant fraction (Russo et al., 1997). In line with the previous findings, testing extracts from AD and DS frontal cortex using ELISA revealed that levels of $\mathrm{A} \beta_{\mathrm{pE} 3}$ and isomerized $\mathrm{A} \beta$ species ending at amino acid 42 were higher than those ending with amino acid 40 (Hosoda et al., 1998; Harigaya et al., 2000). This was further confirmed by the observation that $A \beta_{\mathrm{pE} 3-42}$ constituted $25 \%$ of the total $A \beta_{x-42}$ in plaques of AD brains (Harigaya et al., 2000). It was reported that unmodified $A \beta_{1-40}$ and $A \beta_{1-42}$ can be modified into $A \beta_{\mathrm{pE} 3-42}$ after being injected into rat brain, indicating that rat brains harbor the enzymes required for $\mathrm{N}$-terminal truncation and pyroglutamate formation (Shin et al., 1997). Interestingly, aged dogs, monkeys and bears, which share the same $A \beta$ sequence, harbored $A \beta_{\mathrm{pE} 3-\mathrm{x}}$ in plaques and CAA (Tekirian et al., 1998; Chambers et al., 2010; Hartig et al., 2010). 


\subsubsection{Generation of pyroglutamate-modified $A \beta$}

Formation of $\mathrm{A} \beta_{\mathrm{pE}-\mathrm{x}}$ is a multistep process requiring the removal of the first two amino acids aspartate and alanine in order to expose the $\mathrm{N}$-terminal glutamate at the third position of $\mathrm{A} \beta$. After cleavage of APP by $\beta$ - and $\gamma$-secretase, $A \beta_{1-40 / 42}$ is liberated. Data from our group suggest that the levels of $\mathrm{A} \beta_{\mathrm{PE} 3-\mathrm{x}}$ are inversely linked to the levels of $\mathrm{A} \beta_{1-\mathrm{x}}$ in plaques in APP/PS1KI transgenic mice (Wirths et al., 2010a). In line with this observation, Sevalle et al. suggested that aminopeptidase A can cleave the first amino (aspartate) from A $\beta$ (Sevalle et al., 2009). Interestingly, more than 15 years ago, Saido et al. suggested the presence of hypothetical peptidases cleaving the first two amino acids (Saido et al., 1995). On the other side, $\mathrm{A} \beta_{(\mathrm{E}) 11-\mathrm{x}}$ starting with a glutamate residue is known to be generated by alternative BACE1 cleavage of APP in the TGN (Huse et al., 2002), and is the precursor for $A \beta_{\mathrm{pE} 11-\mathrm{x}}$. After exposure of the glutamate, the enzyme glutaminyl cyclase (QC) catalyses pyroglutamate formation by dehydration of glutamate (Schilling et al., 2004).

\subsubsection{Biochemical properties of pyroglutamate-modified $A \beta$}

The conversion of $A \beta$ into $A \beta_{\mathrm{pE} 3-\mathrm{x}}$ leads to altered biophysical and biochemical characteristics, pointing to changes in aggregation and stability. Saido et al. suggested that $\mathrm{A} \beta_{\mathrm{pE} 3-\mathrm{x}}$ and other modified $\mathrm{A} \beta$ species accumulate because of their limited degradation. The formation of the lactam ring and the loss of two negative charges and one positive charge result in higher hydrophobicity. In addition, the N-terminal pyroglutamate is resistant to degradation by peptidases. As a result, these factors give $A \beta_{\mathrm{pE} 3-\mathrm{x}}$ peptides more stability and aggregation propensity (Saido et al., 1996). He and Barrow reported that $A \beta_{\mathrm{pE} 3-\mathrm{x}}$ peptides show enhanced $\beta$-sheet formation and aggregation propensity in aqueous and hydrophobic media compared to full-length $A \beta$. They suggested that the loss of three charged groups facilitates and stabilizes $\beta$-sheet formation by reducing the level of unfavorable charge repulsion between strands (He and Barrow, 1999). Using a mixture of $A \beta$ species, including $\mathrm{A} \beta_{\mathrm{pE} 3-\mathrm{x}}$ levels mimicking the amount present in $\mathrm{AD}$ patients, abundant globular structures and higher aggregation rates were observed. In contrast, the aggregation propensity using the mixture present in non-demented individuals was significantly lower (Piccini et al., 2005). 
Interestingly, $\mathrm{A} \beta_{\mathrm{pE} 3-\mathrm{x}}$ displayed up to a 250 -fold accelerated formation of aggregates compared to $A \beta_{1-x}$, irrespective of the $C$-terminus of $A \beta$ (Schilling et al., 2006).

Russo et al. claimed that $\mathrm{A} \beta_{\mathrm{pE} 3-40}$ is more toxic for neurons and astrocytes compared to full-length $A \beta_{1-40}$ (Russo et al., 2001). Similarly, another study showed that an A $\beta$ mixture similar to the ones found in the brain of AD patients increased cell membrane permeability, leading to reduced cell survival in neuroblastoma cells (Piccini et al., 2005).

It is worth mentioning that in contrast to what has been described above, some studies have indicated that the secondary structure and toxicity of $A \beta_{\mathrm{pE} 3-40 / 42}$ peptides are similar to that of $A \beta_{1-40 / 42}$ peptides (Tekirian et al., 1999; Wirths et al., 2010b). In agreement, Youssef et al. showed that $A \beta_{1-42}$ and $A \beta_{\mathrm{pE} 3-42}$ exhibited similar effects on neuronal cytotoxicity in primary cortical neurons and on memory impairment after intracerebroventricular injection in wildtype mice (Youssef et al., 2008).

\subsection{Glutaminyl cyclase}

Glutaminyl cyclase (QC) is a metal-dependent acyl transferase that converts glutamine (or alternatively glutamate) into pyroglutamate via the liberation of ammonia (or water) (Schilling et al., 2003). The first isolation of QC was achieved from the latex of the tropical plant Carcia papaya (Messer, 1963). Twenty-four years later, a similar enzyme was detected in mammalian tissues such as brain, pituitary, adrenal medulla and human B lymphocytes (Busby et al., 1987; Fischer and Spiess, 1987). Later, RNA sequences homologous to that in Carcia papaya were identified in Arabidopisis thaliana, Solanum tuberosum and bacteria (Wintjens et al., 2006; Schilling et al., 2007). Although plant and animal QC seem to be similar in terms of their substrate and subcellular localization, cloning of several animal and plant QC showed differences in the primary structures (Pohl et al., 1991; Song et al., 1994; Dahl et al., 2000). This led to the assumption that the two enzymes evolved from different origins (Schilling et al., 2008a). However, both enzymes act by a similar mechanism. The active site of the human QC contains a zinc ion that facilitates the formation of a lactam ring after the removal of water molecule from glutamate (or the ammonia from glutamine) (Schilling et al., 2008a). 


\subsubsection{Physiological functions of glutaminyl cyclase}

QC has a wide range of substrates, which were described and investigated before the isolation of QC itself. QC is involved in the posttranslational maturation of many hormones, such as gonadotropin releasing hormone $(\mathrm{GnRH})$ and thyrotrophin releasing homromone (TRH), as well as neuropeptide like neurotensin and orexin. In addition, other substrates for QC include protein matrixes, such as fibronectin, collagens, and chemokines, such as monocyte chemoattractant proteins 1 to 4 . The presence of pyroglutamate protects proteins such as MCP, GnRH and TRH from degradation by amino peptidases (Sealfon et al., 1997; Van Coillie et al., 1998; Morty et al., 2006). Also, pyroglutamate has been shown to be essential for protein activity. The loss of pyroglutamate leads to reduced protein-receptor interaction, as shown for TRH and MCP (Goren et al., 1977; Van Coillie et al., 1998).

\subsubsection{The role of glutaminyl cyclase in the generation of pyroglutamate-modified A $\beta$}

It has been suggested that cyclization of glutamate to pyroglutamate can occur spontaneously, an assumption that was greatly influenced by the lack of methodological techniques. However, it was shown later that spontaneous cyclization is seventy times slower than enzymatic driven cyclization (Busby et al., 1987; Fischer and Spiess, 1987).

Compelling evidence demonstrates the role of $\mathrm{QC}$ in the generation of $\mathrm{A} \beta_{\mathrm{pE}-\mathrm{x}}$. Incubation of synthetic $A \beta_{3-x}$ with recombinant $Q C$ resulted in the conversion into $A \beta_{\mathrm{PE} 3-\mathrm{x}}$. This reaction is favored under acidic $\mathrm{pH}$ conditions and blocked by the presence of a QC inhibitor (Schilling et al., 2004). Furthermore, QC stimulated $\mathrm{A} \beta_{\mathrm{pE} 3-\mathrm{x}}$ generation in two different cell lines (Cynis et al., 2006; Cynis et al., 2008a). Application of a QC inhibitor suppressed the cyclization reaction to $\mathrm{A} \beta_{\mathrm{pE} 3-\mathrm{x}}$. Interestingly, addition of recombinant $\mathrm{QC}$ into culture media generated only minor amounts of $A \beta_{\mathrm{PE} 3-\mathrm{x}}$. This finding indicates that pyroglutamate $A \beta$ formation is favored within intracellular compartments (Cynis et al., 2008a).

In agreement with in vitro studies, several in vivo reports have supported the role of $\mathrm{QC}$ in the production of $\mathrm{A} \beta_{\mathrm{PE} 3-\mathrm{x}}$. Injection of $\mathrm{A} \beta_{3-40}$ into the cortex of wildtype rats led to significant production of $A \beta_{\mathrm{pE} 3-40}$ within 24 hours, which was inhibited by co-application of a QC inhibitor (Schilling et al., 2008b). Oral administration of a QC inhibitor to Tg2576 and 
TASD-41 transgenic mice reduced $A \beta_{\mathrm{pE} 3-\mathrm{x},} \mathrm{A} \beta_{\mathrm{x}-40}$ and $\mathrm{A} \beta_{\mathrm{x}-42}$ levels. This was accompanied by a reduction in plaque load and gliosis in addition to improvements in contextual fear memory and spatial memory. Similarly, treatment of transgenic Drosophila expressing A $\beta$ transgene, starting with glutamine, with a $Q C$ inhibitor led to reduced $A \beta_{\mathrm{pE3}-42}$ (Schilling et al., 2008c)

(Fig. 11).

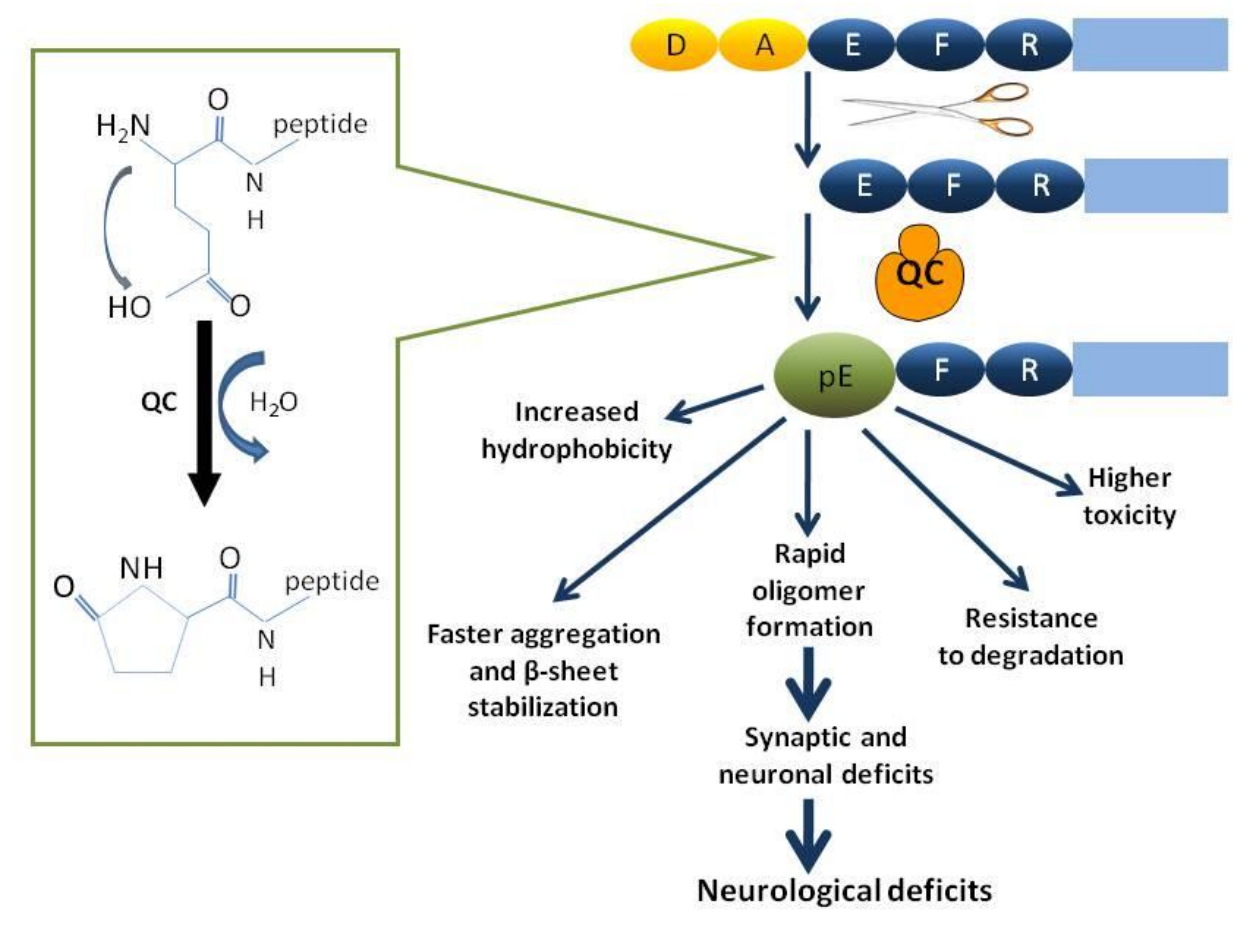

Fig. 11. Generation of pyroglutamate-modified $\mathbf{A} \boldsymbol{\beta}$. The first two $\mathrm{N}$-terminal amino acids, aspartate and alanine, are cleaved off by an unknown mechanism, thereby exposing glutamate at position three of the Nterminus of $A \beta$. Subsequently, glutamate is post-translationally modified into N-terminal pyroglutamate (pE) by dehydration catalysed by glutaminyl cyclase (QC) activity. The novel peptide has altered biochemical properties with severe pathological consequences. The enhanced toxicity is likely due to the higher aggregation propensity and the longer bioavailability of the $\mathrm{A} \beta_{\mathrm{pE} 3-\mathrm{x}}$ oligomers. 


\section{Project I: "Characterization of the 5XFAD mouse model"}

\subsection{Overview}

As mentioned earlier, the 5XFAD mouse model is an AD model overexpressing multiple APP and PS1 mutations which lead to accumulations of $A \beta_{x-42}$ far exceeding those of $\mathrm{A} \beta_{\mathrm{x}-40}$. The significance of this model comes from the early accelerated cerebral amyloid deposition associated with many AD pathological and behavioral hallmarks. It is one of the rare AD models suggested to exhibit neuron loss (Oakley et al., 2006). The major benefit of the 5XFAD mouse model comes from the segregation of the transgenes (APP and PS1) together, a plus for the breeding with other transgenic mice.

The original 5XFAD mice were generated and described on a C57/BLSJL genetic background. 5XFAD mice used in the current study were backcrossed to C57BL/6J genetic background. This was important to facilitate the comparison to other mouse models used in our lab which are maintained on C57BL/6J genetic background. Therefore, it was a prerequisite to characterize the 5XFAD model in detail on the new genetic background especially that previous studies have shown that the genetic background may influence AD pathology (Carlson et al., 1997; Lehman et al., 2003; Lassalle et al., 2008; Glazner et al., 2010). Moreover, it was important to verify the 5XFAD mouse model as an appropriate model to study $\mathrm{A} \beta_{\mathrm{pE} 3-\mathrm{x}}$ in order to use it to study the effect of QC on AD pathology.

In the following manuscript, we performed longitudinal characterization of female 5XFAD mice starting from 3 to 12 months of age. The behavioral performance of the 5XFAD mice was evaluated using a battery of motor and cognitive tasks. In order to evaluate neuron loss, an important hallmark of $\mathrm{AD}$, stereological neuron counting was performed in 12-monthold mice in different brain regions. In addition, age-dependent axonopathy and spinal cord pathology were quantified in different brain regions. Plaque load and intracellular $A \beta$ were also assessed in an age-dependant manner for both the pool of pan $\mathrm{A} \beta$ and $\mathrm{A} \beta_{\mathrm{pE} 3-\mathrm{x}}$.

\section{Aims of Project I}

- To validate the 5XFAD mouse model on the C57BL/6J genetic background.

- To assess unexplored behavioral entities in the 5XFAD mouse model such as anxiety, motor function and explorative activity in an age-dependent manner. 
- To quantify plaque pathology and axonopathy in different brain regions and in the spinal cord.

- $\quad$ To quantify the previously assumed neuron loss using unbiased stereology and study how it relates to the presence of intraneuronal $A \beta$.

- To replicate the memory impairment described in these mice.

- $\quad$ To explore the 5XFAD model as suitable model to study $A \beta_{\mathrm{pE} 3-\mathrm{x} \text {. }}$ 


\subsection{Original publication}

Jawhar, S., Trawicka, A., Jenneckens, C., Bayer, T.A., and Wirths, O. (2010). Motor deficits, neuron loss, and reduced anxiety coinciding with axonal degeneration and intraneuronal Abeta aggregation in the 5XFAD mouse model of Alzheimer's disease. Neurobiology of Aging. doi.org/10.1016/j.neurobiolaging.2010.05.027

\section{Personal contribution}

I was involved in the design of the study. Also, I conducted the behavioral and immunohistochemical experiments. I performed the major parts of stereological analysis. I was responsible for the analysis and interpretation of the behavior, plaque load, sterological and axonopathy data. Besides, I actively participated in writing the manuscript.

\section{Contribution to figures}

I fully contributed to figures $1,2,3,5$ and 6 . In figure 4, I conducted the quantification in the $5^{\text {th }}$ cortical layer. However, I was responsible for the stereology design, statistical analysis and interpretation of the results. In figure 7, I performed the quantification in the spinal cord and pons of the 3-month-old mice. However, I was responsible for the statistical analysis and interpretation of the results. 


\title{
Motor deficits, neuron loss, and reduced anxiety coinciding with axonal degeneration and intraneuronal $\mathrm{A} \beta$ aggregation in the 5XFAD mouse model of Alzheimer's disease
}

\author{
Sadim Jawhar ${ }^{\mathrm{a}}$, Anna Trawicka ${ }^{\mathrm{a}}$, Carolin Jenneckens ${ }^{\mathrm{a}}$, Thomas A. Bayer ${ }^{\mathrm{a}}$, Oliver Wirths ${ }^{\mathrm{a}, *}$ \\ ${ }^{a}$ Division of Molecular Psychiatry, Alzheimer Ph.D., Graduate School, Department of Psychiatry, University of Goettingen, von-Siebold-str. 5, 37075 \\ Goettingen, Germany
}

Received 12 January 2010; received in revised form 10 May 2010; accepted 14 May 2010

\begin{abstract}
In the present report, we extend previous findings in the 5XFAD mouse model and demonstrate that these mice develop an age-dependent motor phenotype in addition to working memory deficits and reduced anxiety levels as demonstrated in an elevated plus maze task. Employing a variety of $\mathrm{N}$ - and $\mathrm{C}$-terminal specific $\mathrm{A} \beta$ antibodies, abundant intraneuronal and plaque-associated pathology, including accumulation of pyroglutamate $\mathrm{A} \beta$, was observed as early as the age of 3 months. Using unbiased stereology, we demonstrate that the 5XFAD mice develop a significant selective neuron loss in layer 5 of the cortex, leaving the overall neuron number of the total frontal cortex and hippocampus unaffected. This observation coincides with the accumulation of intraneuronal A $\beta$ peptides only in cortical Layer 5, but not in CA1, despite comparable APP expression levels. The motor phenotype correlates with abundant spinal cord pathology, as demonstrated by abundant intraneuronal $\mathrm{A} \beta$ accumulation and extracellular plaque deposition. In addition, comparable to the APP/PS1KI mouse model, 5XFAD mice develop an age-dependent axonopathy likely contributing to the behavioral deficits.
\end{abstract}

(C) 2010 IBRO All rights reserved.

Keywords: Motor deficit; Transgenic mice; Working memory; Neuron loss; Intraneuronal Abeta; Axonal degeneration; Amyloid

\section{Introduction}

Alzheimer's disease (AD) is a severe neurodegenerative disorder representing the most frequent form of dementia. It is characterized by the deposition of extracellular plaques composed of the mainly $40-42$ amino acid $\mathrm{A} \beta$ peptide, as well as intracellular neurofibrillary tangles consisting of hyperphosphorylated Tau protein. Over the past years numerous transgenic mouse models of $\mathrm{AD}$ have been generated, reflecting underlying pathological alterations (Duyckaerts et al., 2008). Extracellular $\mathrm{A} \beta$ deposition, as well as therapeutic strategies for plaque removal, have dominated research efforts in $\mathrm{AD}$ during the last years, however, the presence of intraneuronal $\mathrm{A} \beta$ accumulations has recently

\footnotetext{
* Corresponding author. Tel: +4955139 10290; fax: +49 55139 10291.

E-mail address: owirths@uni-goettingen.de.
}

gained in importance. It has been shown that $A \beta 42$ accumulates within neurons in $\mathrm{AD}$-vulnerable brain regions in AD (Gouras et al., 2000) and Down syndrome patients (Gyure et al., 2001) and that neurons from sporadic and familial $\mathrm{AD}$ cases contain increased $\mathrm{A} \beta 42$ levels and an increased $A \beta 42 / A \beta 40$ ratio (Aoki et al., 2008).

The data in $\mathrm{AD}$ mouse models regarding intraneuronal $\mathrm{A} \beta$ are much more consistent and an increasing consensus can be observed during the past years. Early intraneuronal accumulation has been reported in several mouse models, including $\mathrm{APP}_{\mathrm{SDL}} \mathrm{PS}_{\mathrm{M} 146 \mathrm{~L}}$ (Wirths et al., 2001), $\mathrm{APP}_{\mathrm{SL}}$ PS1 $1_{\text {M146L }}$ (Wirths et al., 2002), Tg2576 (Takahashi et al., 2002), 3xTg-AD (Oddo et al., 2003), 5XFAD (Oakley et al., 2006), APP $_{\text {Arc }}$ (Knobloch et al., 2007; Lord et al., 2006), $\mathrm{APP}_{\mathrm{T} 714 \mathrm{I}}$ mice (Van Broeck et al., 2008), in $\mathrm{APP}_{\mathrm{SL}}$ $\mathrm{PS} 1 \mathrm{KI}_{\mathrm{M} 233 \mathrm{~T}, \text { L235P }}$ mice (Casas et al., 2004) in which it was recently shown to correlate with neuron loss (Breyhan et al., 2009; Christensen et al., 2008; Christensen et al., 2010), as 
well as in TBA2 mice expressing pyroglutamate modified A $33-42$ (Wirths et al., 2009).

5XFAD mice expressing human APP with the Swedish, Florida (I716V) and London mutations, together with mutant PS1 (M14 6L, L28 6V) under the control of the murine Thy-1 promoter (Oakley et al., 2006) are particularly interesting due to cointegration of the transgenes, enabling easy generation of multitransgenic AD mouse models. These mice were generated on a B6/SJL-hybrid background and have been backcrossed with $\mathrm{C} 57 \mathrm{~B} 16 / \mathrm{J}$ mice for five generations to facilitate comparison with other models which are mostly kept on the C57B16 genetic background.

In the present report, we extent previous findings in the 5XFAD mouse model and demonstrate that they develop an age-dependent motor phenotype in addition to working memory deficits in an alternation task and reduced anxiety levels as shown in the elevated plus maze task. Quantification of cortical layer 5 neurons in 12 month old 5XFAD mice by means of design-based stereology confirmed the previously assumed loss of neurons in this brain regions (Oakley et al., 2006; Ohno et al., 2007), however, the overall neuron number in frontal cortex and hippocampal CA1 layer was unchanged compared with age-matched wild-type littermates. This observation coincides with the accumulation of intraneuronal $\mathrm{A} \beta$ peptides only in cortical Layer 5, but not in CA1. The motor phenotype correlates well with abundant spinal cord pathology, including intraneuronal $\mathrm{A} \beta$ accumulation and development of an agedependent axonal degeneration.

\section{Methods}

\subsection{Transgenic mice}

The generation of 5XFAD mice (Tg6799) has been described previously (Oakley et al., 2006). In brief, 5XFAD overexpress the 695 amino acids isoform of the human amyloid precursor protein (APP695) carrying the Swedish, Florida, and London mutations under the control of the murine Thy-1-promoter. In addition, human presenilin-1 (PS1) carrying the M14 6L and L28 6V mutations mice is expressed also under the control of the murine Thy-1-promoter. Five male mice on a $\mathrm{C} 57 \mathrm{Bl} / 6$ x SJL genetic background were obtained from Jackson Laboratories (strain: B6SJL-Tg(APPSwFILon, PSEN1*M146L*L286V)6799Vas/J) and backcrossed for 5 generations to $\mathrm{C} 57 \mathrm{Bl} / 6 \mathrm{~J}$ wildtype (WT) mice to obtain an incipient congenic line on a $\mathrm{C} 57 \mathrm{Bl} /$ $6 \mathrm{~J}$ Genetic background. C57B16/J WT mice were used as control animals. All animals were handled according to German guidelines for animal care. Only female mice were used in the current study.

\subsection{Clasping test}

To test clasping behavior, mice were suspended by the tail for $30 \mathrm{sec}$ to initiate the clasping phenotype. A score was given on a scale from 0 to three, where 0 represented no clasping, 1 = forepaws clasping, 2 = forepaws and one hind paw clasping, and 3 = all paws clasping (Miller et al., 2008). Unpaired $t$-test was used to compare the clasping score between the 12 mo old 5XFAD mice and their WT littermates.

\subsection{Balance beam task}

Balance and general motor function were assessed using the balance beam task. A $1 \mathrm{~cm}$ dowel beam is attached to two support columns $44 \mathrm{~cm}$ above a padded surface. At either end of the $50 \mathrm{~cm}$ long beam a $9 \times 15 \mathrm{~cm}$ escape platform is attached. The animal is placed on the center of the beam and released. Each animal is given three trials during a single day of testing. The time the animal remained on the beam is recorded and the resulting latencies to fall of all three trials are averaged. If an animal remains on the beam for whole 60-second trial or escapes to one of the platforms, the maximum time of $60 \mathrm{sec}$ is recorded (Arendash et al., 2001; Wirths et al., 2008). The following numbers of animals were analyzed in this task: WT: $n=11$ (3 $\mathrm{m}), \mathrm{n}=13(6 \mathrm{~m}), \mathrm{n}=17(9 \mathrm{~m}), \mathrm{n}=18$ (12 m); 5XFAD: $\mathrm{n}=7(3 \mathrm{~m}), \mathrm{n}=8(6 \mathrm{~m}), \mathrm{n}=10(9 \mathrm{~m}), \mathrm{n}=9(12 \mathrm{~m})$.

\subsection{String suspension task}

This test was performed essentially as described previously (Wirths et al., 2008). In brief, the animals are permitted to grasp the string by their forepaws and are released. A rating system from 0 to 5 is used during the single 60 -sec trial to assess each animals' performance in this task: $0=$ unable to remain on the string; 1 = hangs only by fore- or hind paws; 2 = same as for 1 , but attempts to climb onto string; $3=$ sits on string and is able to hold balance; $4=$ four paws and tail around string with lateral movement; $5=$ escape. The following numbers of animals were analyzed in this task: WT: $\mathrm{n}=11(3 \mathrm{~m}), \mathrm{n}=12(6 \mathrm{~m}), \mathrm{n}=17(9 \mathrm{~m})$, $\mathrm{n}=17(12 \mathrm{~m})$; 5XFAD: $\mathrm{n}=7(3 \mathrm{~m}), \mathrm{n}=8(6 \mathrm{~m}), \mathrm{n}=10$ $(9 \mathrm{~m}), \mathrm{n}=9(12 \mathrm{~m})$.

\subsection{Cross-maze}

Spontaneous alternation rates were assessed using a cross-maze built from black plastic material which had four arms arranged in $90^{\circ}$ position extending from a central space measuring $8 \times 8 \mathrm{~cm}$ (arm sizes: $30 \mathrm{~cm}$ length, $8 \mathrm{~cm}$ width, and $15 \mathrm{~cm}$ height). This test is based on the same principle and protocol as the Y-maze. During 10 minute test sessions, each mouse was randomly placed in one arm and allowed to move freely through the maze. Alternation was defined as successive entries into the four arms in overlapping quadruple sets (e.g., 1, 3, 2, 4 or 2, 3, 4, 1 but not 1, 2, $3,1)$. The alternation percentage was calculated as the percentage of actual alternations to the possible number of arm entries. To diminish odor cues, the maze was cleaned with $70 \%$ ethanol solution. The following number of animals was analyzed in this task: WT: $n=11(3 \mathrm{~m}), \mathrm{n}=13$ 
(6 m), $\mathrm{n}=18(9 \mathrm{~m}), \mathrm{n}=18(12 \mathrm{~m}) ;$ 5XFAD: $\mathrm{n}=7(3 \mathrm{~m})$, $\mathrm{n}=12(6 \mathrm{~m}), \mathrm{n}=10(9 \mathrm{~m}), \mathrm{n}=9(12 \mathrm{~m})$.

\subsection{Elevated plus maze}

The elevated plus maze has the shape of a "+" with two alternate open and two alternate closed arms extending from a central platform. The whole maze is raised $75 \mathrm{~cm}$ above the floor. The open and enclosed arms of the plus maze generate exploratory behavior and the avoidance of elevated open arms is an indication of the intensity of anxiety. During the test the mouse is placed onto the center field and is allowed to explore the maze for $5 \mathrm{~min}$. Anxiety can be measured by the time spent in the open arms, with lower anxiety levels corresponding to more time spent in open arms (Karl et al., 2003). The percentage of the time spent in the open arms to the overall time and the ratio of the open arms to the total arms entries were measured using an automatic video tracking system (VideoMot2, TSE-Systems, Bad Homburg, Germany). The following number of animals was analyzed in this task: WT: $\mathrm{n}=10(3 \mathrm{~m})$, $\mathrm{n}=13(6 \mathrm{~m}), \mathrm{n}=12(9 \mathrm{~m}), \mathrm{n}=17(12 \mathrm{~m}) ;$ 5XFAD: $\mathrm{n}=$ $7(3 \mathrm{~m}), \mathrm{n}=12(6 \mathrm{~m}), \mathrm{n}=10(9 \mathrm{~m}), \mathrm{n}=9(12 \mathrm{~m})$.

\subsection{Open Field}

The open field test was used to asses both exploratory behavior and locomotor activity. The mice were tested using an open field box made of gray plastic with $50 \times 50 \mathrm{~cm}$ surface area and $38 \mathrm{~cm}$-high walls. Monitoring was done by an automated tracking system (VideoMot2, TSE-Systems, Bad Homburg, Germany). The behavioral parameters registered during 5-minute sessions were (i) the percentage of time spent in the central part $(20 \times 20 \mathrm{~cm})$ versus total time (ii) total traveled distance.

\subsection{Immunohistochemistry on paraffin sections}

Mice were transcardially perfused with $4 \%$ PFA in PBS and brains were carefully dissected. Post fixation was carried out in $4 \%$ buffered formalin at $4{ }^{\circ} \mathrm{C}$ before the tissue was embedded in paraffin. Immunohistochemistry was performed on $4 \mu \mathrm{m}$ sagital paraffin sections as described previously (Wirths et al., 2001). In brief, sections were deparaffinized in xylene and rehydrated in a series of ethanol. After treatment with $0.3 \% \mathrm{H}_{2} \mathrm{O}_{2}$ in PBS to block endogenous peroxidases, antigen retrieval was achieved by boiling sections in 0.01-M citrate buffer $\mathrm{pH} 6.0$, followed by 3 -minute incubation in $88 \%$ formic acid. Nonspecific binding sites were blocked by treatment with skim milk and fetal calf serum in PBS, before the addition of the primary antibodies. The following antibodies were used: 4G8 (A $\beta 17-14$, 1:10,000, Covance, Princeton, USA), NF200 (200 kDa-neurofilament, 1:1,000, Millipore, Schwalbach, Germany), 2-48 (A $\beta 3 \mathrm{pE}-\mathrm{x}, 1: 100$, Synaptic Systems, Göttingen, Germany, (Wirths et al., 2010)), A $\beta[\mathrm{N}]$ (1:2,000, IBL, Hamburg, Germany), 692 against $A \beta$ (1:500) and 23850 against human APP (1:500) (Wirths et al., 2007), G2-10 (A $\beta 40,1: 500$,
Millipore, Schwalbach, Germany), ubiquitin (1:500, Dako, Glostrup, Denmark). Primary antibodies were incubated overnight in a humid chamber at room temperature followed by incubation with biotinylated secondary antibodies (Dako, Glostrup, Denmark). Staining was visualized using the ABC method using a Vectastain kit (Vector Laboratories, Burlingame, USA) and diaminobenzidine (DAB) as chromogen providing a reddish-brown color. Counterstaining was carried out with hematoxylin.

\subsection{Quantification of $A \beta$ plaque load}

Extracellular A $\beta$ load (4G8, 2-48) was evaluated in cortex and hippocampus using an Olympus BX-51 microscope equipped with an Olympus DP-50 camera and the ImageJ software (V1.41, NIH, USA). Serial images of $40 \times$ magnification (hippocampus) and 100x (cortex) were captured on six sections per animal which were $30-\mu \mathrm{m}$ afar from each other. Using ImageJ the pictures were binarized to 16-bit black and white images and a fixed intensity threshold was applied defining the DAB staining. Measurements were performed for a percentaged area covered by DAB staining (Breyhan et al., 2009). The following number of animals was used in this analysis: 5XFAD: $n=4(3 \mathrm{~m})$, $\mathrm{n}=4(6 \mathrm{~m}), \mathrm{n}=5(12 \mathrm{~m})$. One-way ANOVA followed by unpaired $t$-test was used to compare age-dependent changes in plaque load for each antibody.

\subsection{Quantification of neuron numbers using design- based stereology}

Twelve months old 5xFAD $(n=5)$ and age-matched WT mice $(\mathrm{n}=5)$ were anesthetized and transcardially perfused with $4 \%$ paraformaldehyde as previously described (Christensen et al., 2008). The brains were carefully removed from the skull, postfixed for $2 \mathrm{~h}$ and dissected. Stereological analysis was performed as previously described (Casas et al., 2004; Schmitz et al., 2004). Briefly, the left brain hemispheres were cryoprotected in $30 \%$ sucrose, quickly frozen and cut frontally into entire series of $30-\mu \mathrm{m}$ thick sections on a cryostat (microM HM550, Thermo, Fisher Scientific, Walldorf, Germany). Every 10th section was systematically sampled, stained with cresyl violet and used for stereological analysis of the neuron number in the CA1, overall frontal cortex and fifth cortical layer. The hippocampal cell layer CA1 (bregma -1.22 to $-3.80 \mathrm{~mm}$ ), overall cortex (bregma 3.08-0.26 $\mathrm{mm}$ ) and fifth cortical layer (bregma -0.1 to -3.88 ) were delineated on cresyl violet-stained sections. Using a stereology workstation (Olympus BX51 with a motorized specimen stage for automatic sampling, StereoInvestigator 7 [Microbrightfield, Williston, VT, USA]) and a $100 \times$ oil lens (NA = 1.35), neuronal nuclei were sampled systematic uniformly random using optical disector probes, and the total number of neurons was subsequently estimated by the fractionator method using a $2 \mu \mathrm{m}$ top guard zone (Schmitz and Hof, 2005; West et al., 1991; West, 2002). Table 1 summarizes 
Table 1

Details of the counting procedure used to evaluate neuron numbers

\begin{tabular}{|c|c|c|c|c|c|c|c|c|}
\hline Region counted & Obj. & Av. No. sections & $\mathrm{B}\left(\mu \mathrm{m}^{2}\right)$ & $\mathrm{H}(\mu \mathrm{m})$ & $\mathrm{t}(\mu \mathrm{m})$ & $\Sigma \mathrm{OD}$ & $\Sigma \mathrm{Q}^{-}$ & $\mathrm{CE}_{\text {pred }}[\mathrm{n}]$ \\
\hline CA1 pyramidal neurons & $100 \times$ & 9 & 196 & 5 & 10.4 & 301.3 & 477.8 & 0.046 \\
\hline Frontal cortex & $100 \times$ & 9 & 625 & 6 & 11.7 & 219.3 & 348.2 & 0.055 \\
\hline Cortex layer 5 neurons & $100 \times$ & 11 & 625 & 6 & 10.7 & 135 & 228.8 & 0.068 \\
\hline
\end{tabular}

Av. no sections, average number of sections used; B and H, base and height of the optical disectors; Obj, objective used t, measured average section thickness after histological processing; $\Sigma$ OD, average sum of optical disectors used; $\Sigma \mathrm{Q}^{-}$, average number of neurons counted; $\mathrm{CE}_{\text {pred }}[\mathrm{n}]$, average predicted coefficient of error of the estimated total number of neurons.

the details of the counting procedures. Unpaired $t$-test was used to compare the difference in neurons number between 12 month old 5XFAD mice and their WT littermates.

\subsection{Axonal degeneration}

To quantify axonal degeneration, paraffin-sections of brain and spinal cord were double-stained for neurofilaments (NF200, DAB) and $\mathrm{A} \beta$ (4G8, Histogreen) and the number of nonplaque-associated spheroids was quantified in both pons (sagittal sections, starting at bregma $0.36 \mathrm{~mm}$ ) (Paxinos and Franklin, 2001) and spinal cord (cross-sections of gray matter of the medial part of the cervical spinal cord with a random start). Serial images of $100 \times$ magnification were captured on six sections per animal which were 30- $\mu \mathrm{m}$ afar from each other. For quantification, the meander scan option of StereoInvestigator 7 (Microbrightfield, Williston, VT, USA) was used. The following number of animals was used in this analysis: 5XFAD: $n=4(3 \mathrm{~m}), \mathrm{n}=$ $4(6 \mathrm{~m}), \mathrm{n}=5(12 \mathrm{~m})$. One-way ANOVA followed by unpaired $t$-test was used to compare age-dependent changes in axonal spheroids.

\subsection{Statistical analysis}

Differences between groups were tested with either oneway or two-way analysis of variance (ANOVA) followed by Bonferroni post-tests or unpaired $t$-tests. All data were given as mean \pm SEM. Significance levels were given as follows: $* * * p<0.001$; $* * p<0.01$; $* p<0.05$. All calculations were performed using GraphPad Prism version 4.03 for Windows (GraphPad Software, San Diego, USA).

\section{Results}

\subsection{Phenotypical characterization}

5XFAD mice showed a reduced body weight in comparison with their WT littermates. Analysis by Two-way ANOVA revealed a significant genotype effect starting at 9 months of age ( $p<0.05$, Fig. 1A). In addition, 5XFAD mice showed a characteristic clasping phenotype presented by an unusual simultaneous retraction of both fore- and hind paws. Quantitative measurement of this behavior at $12 \mathrm{mo}$ of age, by suspending the animals by their tail and evaluating the clasping score, revealed a significant increase in the clasping score in the 5XFAD mice $(p<0.001$, Fig. 1B).
Sensory-motor abilities were analyzed using two different tasks. The balance beam measures the ability of the mice to remain on a thin wooden dowel. Whereas 3 and 6 mo old 5XFAD mice did not show any significant impairment in this task compared with their WT littermates, 9 mo old 5XFAD mice started to show a decreased latency to fall $(p<0.05)$ which became more significant by 12 mo of age $(p<0.001$, Fig. 1C). The second sensory-motor task used was the string suspension paradigm which measures the ability of a mouse to hold on a wire using its forepaws. 5XFAD mice showed poorer performance compared with the WT control mice at both 9 and 12 mo of age (both $p<$ 0.001 ), whereas younger mice at 3 and 6 mo of age did not show impairment ( $p>0.05$, Fig. 1D).

\subsection{Age-dependent working memory impairment in 5XFAD mice}

The cross maze was used to measure the alternation as an indicator of spatial working memory impairment. Analysis of the alternation percentage did not show any noticeable problems at the age of 3 mo $(p>0.05)$, however, it revealed a significant impairment in the working memory in 6 mo old 5XFAD mice which became more significant in 9 and 12 mo old mice $(6 \mathrm{~m}, p<0.05 ; 9 \mathrm{~m}, p<0.01 ; 12 \mathrm{~m}, p<0.01$ ) (Fig. 1E). The reduced alternation percentage was obviously not due to a decrease in overall explorative behavior as 5XFAD mice showed the same number of total arm entries in comparison with their WT littermates at any analyzed time point (Fig. 1F).

\subsection{Age-dependent increase of plaque-associated $A \beta$ in cortex and hippocampus}

Sagittal brain sections of 5XFAD mice at 3, 6, and $12 \mathrm{mo}$ of age (Fig. 2) were stained with two different $\mathrm{A} \beta$ antibodies detecting either a central $A \beta$ epitope (4G8, $A \beta 17-24)$ or $\mathrm{N}$-terminal truncated $\mathrm{A} \beta$ peptides starting with a pyroglutamate at Position $3(2-48 ; \mathrm{A} \beta \mathrm{N} 3 \mathrm{pE})$. Plaque load was quantified in the frontal cortex and the hippocampus as described earlier (Christensen et al., 2008) (Fig. 2A-F). Using the 4G8 antibody a continuous increase in $\mathrm{A} \beta$ plaque load was noted in both cortex (3 m: $1.89 \pm 0.57 \% ; 6 \mathrm{~m}$ : $5.23 \pm 1.09 \% ; 12 \mathrm{~m}: 9.72 \pm 0.91 \%)$ and hippocampus $(3$ $\mathrm{m:} 0.78 \pm 0.11 \% ; 6 \mathrm{m:} 3.32 \pm 0.34 \% ; 12 \mathrm{~m}: 8.03 \pm 1.04 \%$, Fig. 2A-C). The same holds true for quantification using the 
A

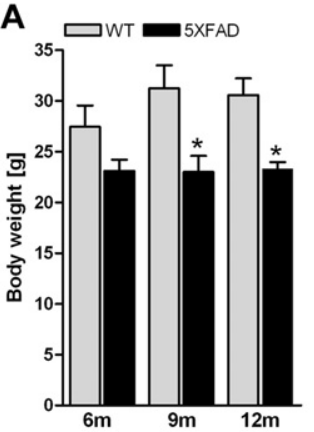

B
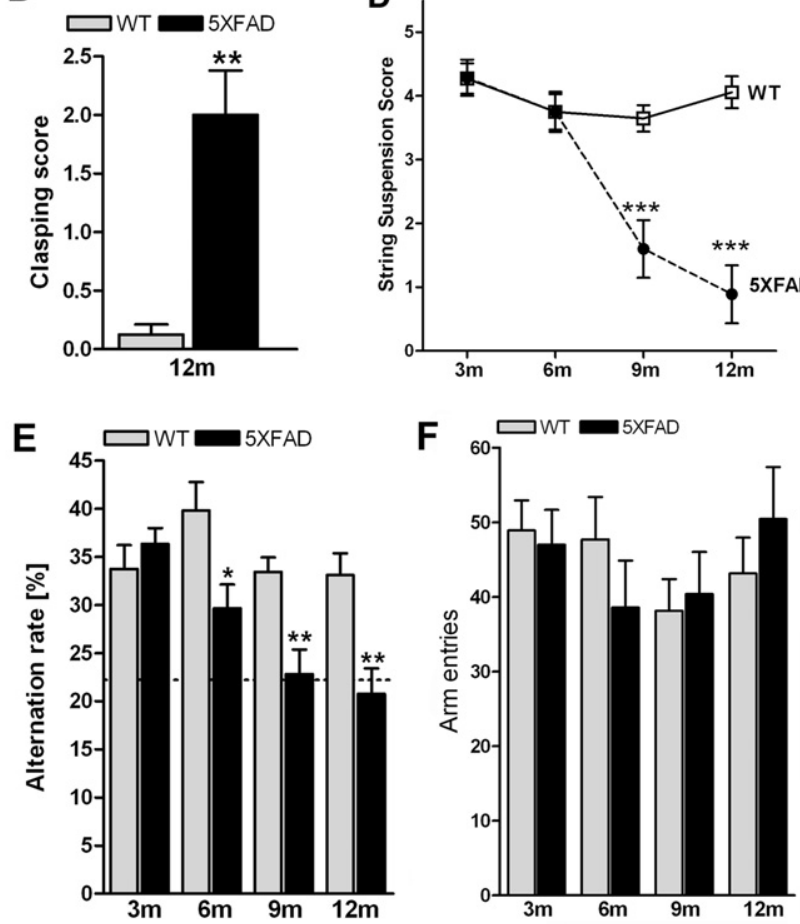

Fig. 1. 5XFAD mice show age-dependent changes in body weight (A) Analysis of clasping behavior showed a significant difference between 5XFAD mice and WT littermates at 12 months of age (B). Analysis of motor performance revealed deficits in the balance beam (C) and the string suspension task (D) starting at 9 months of age. Evaluation of working memory using the cross-maze demonstrated reduced alternation rates starting from 6 months of age. The dotted line represents chance level $(\mathrm{E})$. This reduction is not due reduced explorative behavior because there was no difference in the total arm entries between the 5XFAD and their WT littermates $(\mathrm{F})$. All error bars represent SEM $* * * p<0.001, * * p<0.01 ; * p<0.05$

2-48 antibody detecting pyroglutamate- $\mathrm{A} \beta$, which also shows a continuous significant increase in both cortex $(3 \mathrm{~m}$ : $0.09 \pm 0.03 \%$; $6 \mathrm{~m}: 0.83 \pm 0.1 \% ; 12 \mathrm{m:} 3.53 \pm 0.88 \%)$ and hippocampus ( $3 \mathrm{~m}: 0.05 \pm 0.03 \% ; 6 \mathrm{~m}: 1.64 \pm 0.28 \%$; $12 \mathrm{~m}: 3.1 \pm 0.47 \%$, Fig. 2D-F).

\subsection{Decreased anxiety levels but normal locomotor behavior in $5 X F A D$ mice}

The elevated plus maze was used to study anxiety levels in 5XFAD mice at 3, 6, 9, and 12 mo of age. 5XFAD mice showed an age-dependent decreased anxiety phenotype which was indicated by spending more time in the open arms starting from 6 mo of age $(6,9,12 \mathrm{~m}: p<0.001)$ (Fig. $3 \mathrm{C})$. This was confirmed by calculating the ratio of open arm entries to total arm entries which revealed significant higher ratios in 6, 9, and 12 mo old 5XFAD mice compared with WT animals $(p<0.001)$ (Fig. 3D). In addition, exploratory and spontaneous locomotor activity of the 5XFAD mice was compared with WT mice at 9 and 12 mo of age in the open-field paradigm. No significant difference in the covered distance (Fig. 3F) or the speed (not shown) between WT and 5XFAD mice was noted during the 5 min trial $(p>$ 0.05 ). Interestingly, 12 mo old 5XFAD mice spent more time in the center of the maze in comparison with their WT littermates $(p<0.001)$ reflecting reduced anxiety and confirming the findings from the elevated plus maze experiment (Fig. 3E).

\subsection{No overt hippocampal and cortical neuron loss in 12 month old 5XFAD mice}

The number of neurons was quantified in the CA1 region of the hippocampus and the frontal cortex in 12 mo old 5XFAD and WT mice by design-based stereology. CA1 was defined from bregma -1.22 to -3.80 and the frontal cortex was defined from bregma 3.08 to 0.26 . Stereological quantification of the pyramidal neurons of the CA1 layer showed no significant difference between the numbers of neurons of WT $(261,654 \pm 16,594)$ and 5XFAD mice $(221,848 \pm$ 12,088) (Fig. 4A-C). The same holds true for the neuron number of the frontal cortex where no significant difference between WT $(2,031,081 \pm 301,914)$ and 5XFAD $(1,867,068 \pm$ 154,977) mice could be established (Fig. 4D-F).

\subsection{Loss of neurons in cortical Layer 5 correlates with intraneuronal $A \beta$ accumulation}

A loss of neurons in the Layer 5 of the cortex has been previously assumed, however, quantitative data were lacking so far (Oakley et al., 2006; Ohno et al., 2007). Therefore, cortical layer 5 neurons were counted in 12 mo old 5XFAD and WT mice by design-based stereology and a significant loss of these neurons was found in 5XFAD $(320,896 \pm 44,205)$ compared with WT mice $(518,451 \pm$ 30,133; $p<0.01$ ) (Fig. 4G-I). Qualitative immunostainings using an antiserum against human APP revealed strong immunoreactivity in deep cortical layers (Fig. 5A-B), as well as in the CA1-CA3 regions of the hippocampal formation (Fig. 5F-G). Staining with antibodies detecting various forms of $\mathrm{A} \beta$ peptides revealed strong intraneuronal immunoreactivity in cortical layer 5 neurons in 3 mo old 5XFAD mice (Fig. 5C-E), which decreased with aging and was almost absent in 12 mo old 5XFAD mice (not shown). No intraneuronal $\mathrm{A} \beta$ could be detected in the hippocampal formation of 3,6 , or 12 mo old 5XFAD mice (Fig. 5H). Interestingly, using an $A \beta 40$-specific antibody (G2-10) nu- 

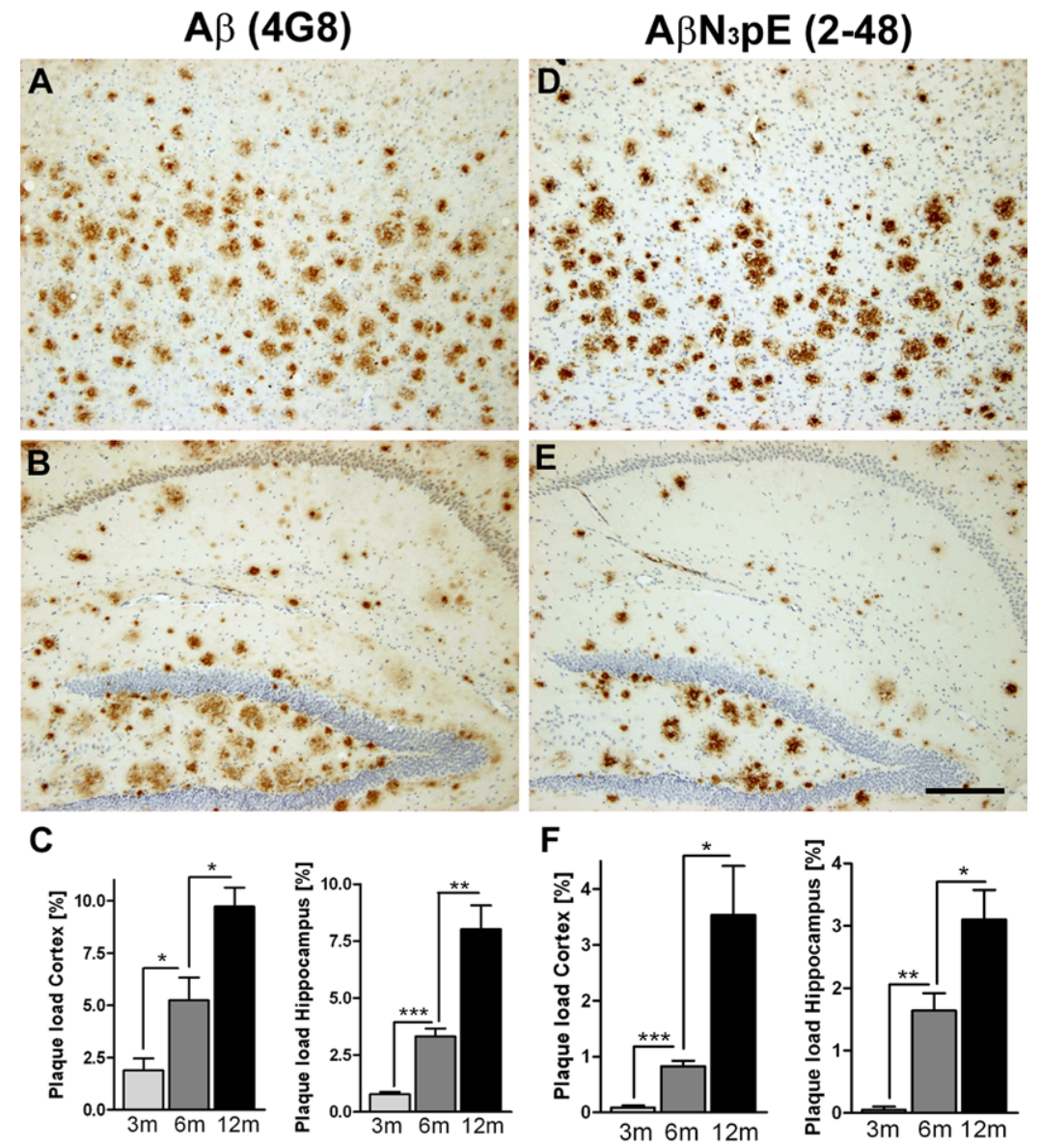

Fig. 2. Plaque-load quantification in cortex and hippocampus showed an age-dependent increase from 3 to 6-to 12-month-old mice using antibodies that detect pan A $\beta$ (4G8, A-C) and pyroglutamate-modified-A $\beta$ (2-48, D-F). Representative images of 6-month-old mice were shown. All error bars represent SEM *** $p<0.001, * * p<0.01 ; * p<0.05$. Scale bar: $200 \mu \mathrm{m}$.

merous plaque-associated, as well as plaque-independent A $\beta 40$-positive axonal swellings could be detected in three-, six- and 12 month old 5XFAD mice. In addition, A $\beta 40$ positive neuritic processes were detected at later stages (Fig. $5 \mathrm{I}-\mathrm{K})$.

\subsection{A $\beta$ pathology in the spinal cord of SXFAD mice}

As human APP is expressed under the control of the neuron-specific murine Thy-1-promoter, it is present in both brain and spinal cord. Using an antiserum against human APP, abundant APP immunoreactivity was detected in spinal cord motor neurons, as well as in neuronal processes of the dorsal column (Fig. 6A,B,E). Quantification of extracellular $A \beta$ plaques in the spinal cord revealed a significant age-dependent increase (Fig. 6D). In addition, intraneuronal $\mathrm{A} \beta$ accumulation in spinal cord motor neurons could be demonstrated using different $\mathrm{A} \beta$ antibodies (Fig. $6 \mathrm{C}, \mathrm{F}-\mathrm{H}$ ).

\subsection{Axonal degeneration in 5XFAD mice}

Staining with antibodies against APP, ubiquitin (not shown) and NF200 (a neurofilament subunit) disclosed marked axonal swellings in the brain and the spinal cord, mainly decorating extracellular plaques in the form of dystrophic neurites (Fig. 5B, 7E). However, larger axonal spheroids were also present independent from plaques. These axonal dilatations were present in many brain regions already at 3 mo of age, such as cortex, hippocampus, midbrain, and hindbrain regions like medulla or pons which increased in an age-dependent manner. To examine whether the motor deficits correlate with the axonal defects, a detailed analysis of the spinal cord revealed that the axonal dilatations were present mainly in the gray matter in addition to some spheroids located at the border of gray and white matter.

To quantify plaque-independent axonal spheroids, double stainings using 4G8 and NF200 were performed. Quantification was carried out in the pons (Fig. 7A-C) and the gray matter of the spinal cord (Fig. 7E-G). One-way ANOVA followed by unpaired $t$-test revealed a significant increase in plaque independent axonal spheroids between 3 and 6 mo of age in the pons $(p<0.05)$, as well as the spinal cord $(p<0.01)$, in which a further significant increase between 6 and 12 mo of age was detected $(p<0.001)$, while in the pons no significant additional increase was noted between these time points (Fig. 7D,G). 

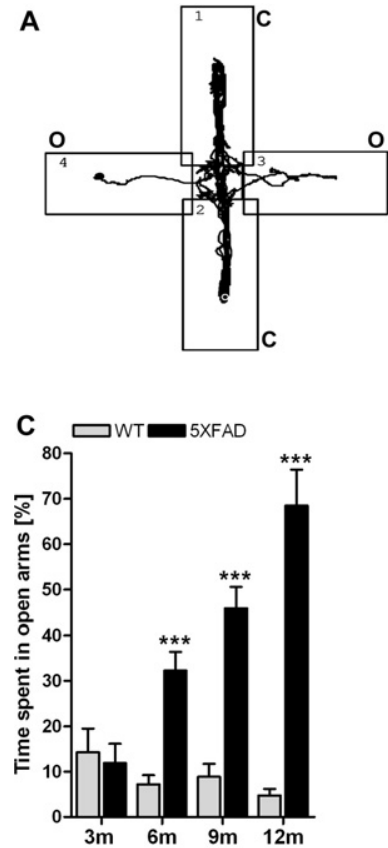

E

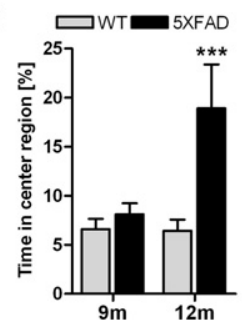

B
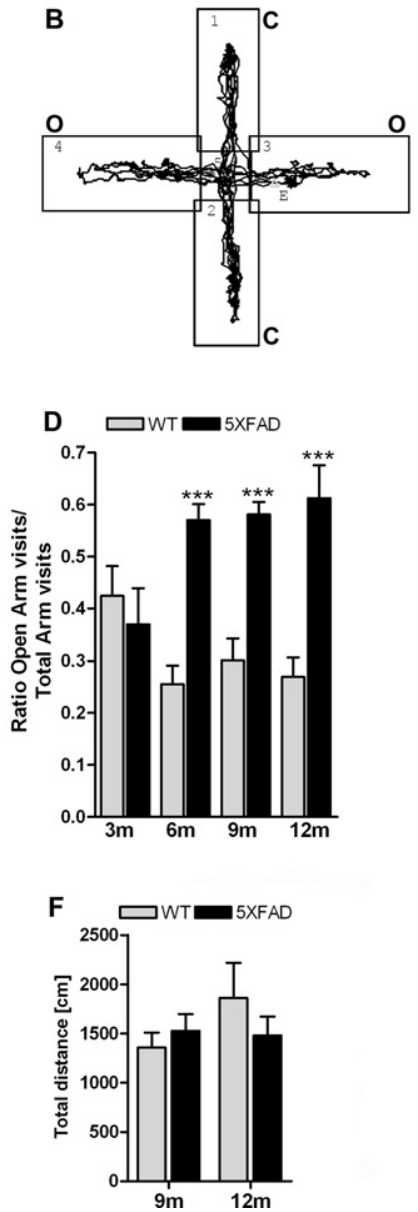

Fig. 3. Elevated-plus maze revealed reduced anxiety in 5XFAD mice. An example of the recorded path of WT mice (A) and 5XFAD mice (B) in the elevated-plus maze. The time spent in the open arms (o) was significantly higher in 5XFAD mice compared with their WT littermates starting from 6 months of age (C). The ratio of open arm visits to total visits showed the same pattern (D). 12-months-old 5XFAD mice spent more time in the center of the open field in mice compared with WT littermates (E). However, there was no difference in the total traveled distance between $5 \mathrm{XFAD}$ and WT mice. All error bars represent SEM ***p $<0.001, * * p<$ $0.01 ; * p<0.05$.

\section{Discussion}

The 5XFAD mouse model represents a double transgenic APP/PS1 mouse line coexpressing five familial Alzheimer disease (5XFAD) mutations that are inherited together and lead to accelerated plaque formation and increased $A \beta 42$ levels. It was previously described as one of the few $A D$ models showing several $\mathrm{AD}$ hallmarks, including neuron loss (Oakley et al., 2006). The aim of the present work was to perform a detailed characterization of the 5XFAD mouse model on a C57/BLJ genetic background, providing a detailed neuropathological, behavioral and stereological analysis in an age-dependent manner.

The 5XFAD model has been reported to develop plaque deposition starting already at the age of 2 mo (Oakley et al., 2006). Two pools of $A \beta$ plaques were analyzed at different

time points which were either total $\mathrm{A} \beta$ (detected by $4 \mathrm{G} 8$ ) and pyroglutamate-modified $A \beta$ (detected by $2-48$ ). Both total $\mathrm{A} \beta$ and the pyroglutamate-modified $\mathrm{A} \beta$ pools showed a continuous increase with age in cortex and hippocampus. The importance of pyroglutamate-modified $\mathrm{A} \beta$ arises from its characteristics, such as higher aggregation propensity (He and Barrow, 1999; Schilling et al., 2006), stronger hydrophobicity (Kuo et al., 1997), and increased toxicity compared with full-length $\mathrm{A} \beta$ (Russo et al., 2002) making it a potential target to treat AD. 5 XFAD mice display a progressive neurological phenotype which is characterized by growth retardation, an abnormal clasping phenotype and motor impairment. 12-Months-old 5XFAD mice showed an abnormal extension reflex by retracting hind- and fore-paws simultaneously when suspended by the tail. This abnormal clasping-pattern has been described in several AD models, including mice transgenic for human four-repeat Tau (Probst et al., 2000), human apolipoprotein E4 (ApoE4) (Tesseur et al., 2000), as well as APP/PS1KI (Wirths et al., 2007; Wirths et al., 2008), which all showed signs of axonopathy. In agreement with the clasping behavior, the motor phenotype started to become prominent in 5XFAD mice at 9 mo of age when tested in the balance beam and string suspension tasks. Motor impairments, including rigidity and impairments in gait or posture have also been repeatedly reported in AD patients (O'Keeffe et al., 1996; Pettersson et al., 2005; Scarmeas et al., 2004; Scarmeas et al., 2005; Wirths and Bayer, 2008), as well as other APP transgenic mouse models, including APP single transgenic mice (Le Cudennec et al., 2008; Lee et al., 2004), APP/PS1 (Ewers et al., 2006) or APP/PS1KI mice (Wirths et al., 2008). The assessment of motor impairment is a highly relevant issue, as some reference memory tasks like the Morris water maze are severely influenced by any disturbance in motor performance, leading to erroneous results.

The motor deficits seen in the 5XFAD mice correlate with the formation of axonal spheroids present in the brain and spinal cord, which represents the most prominent histological evidence of central nervous system axonopathy in these mice. Axonal swellings have been previously reported in other APP transgenic mouse models to a variable extent (Stokin et al., 2005; Wirths et al., 2006; Wirths et al., 2007) and are also present in Tau (Probst et al., 2000; Spittaels et al., 1999) or ApoE-based (Tesseur et al., 2000) AD transgenic mouse models. The presence of intraneuronal $\mathrm{A} \beta$ within spinal cord motor neurons might lead to axonal transport impairment, as it has been shown that $\mathrm{A} \beta 42$ accumulates in lumbar motor neurons of patients suffering from amyotrophic lateral sclerosis (ALS) (Calingasan et al., 2005), a disease in which axonal transport deficits are clearly implicated (reviewed in (Chevalier-Larsen and Holzbaur, 2006)). We hypothesize that the accumulation of intraneuronal $\mathrm{A} \beta$ in motor neurons in the 5XFAD mouse model contributes to impaired axonal transport, which is 

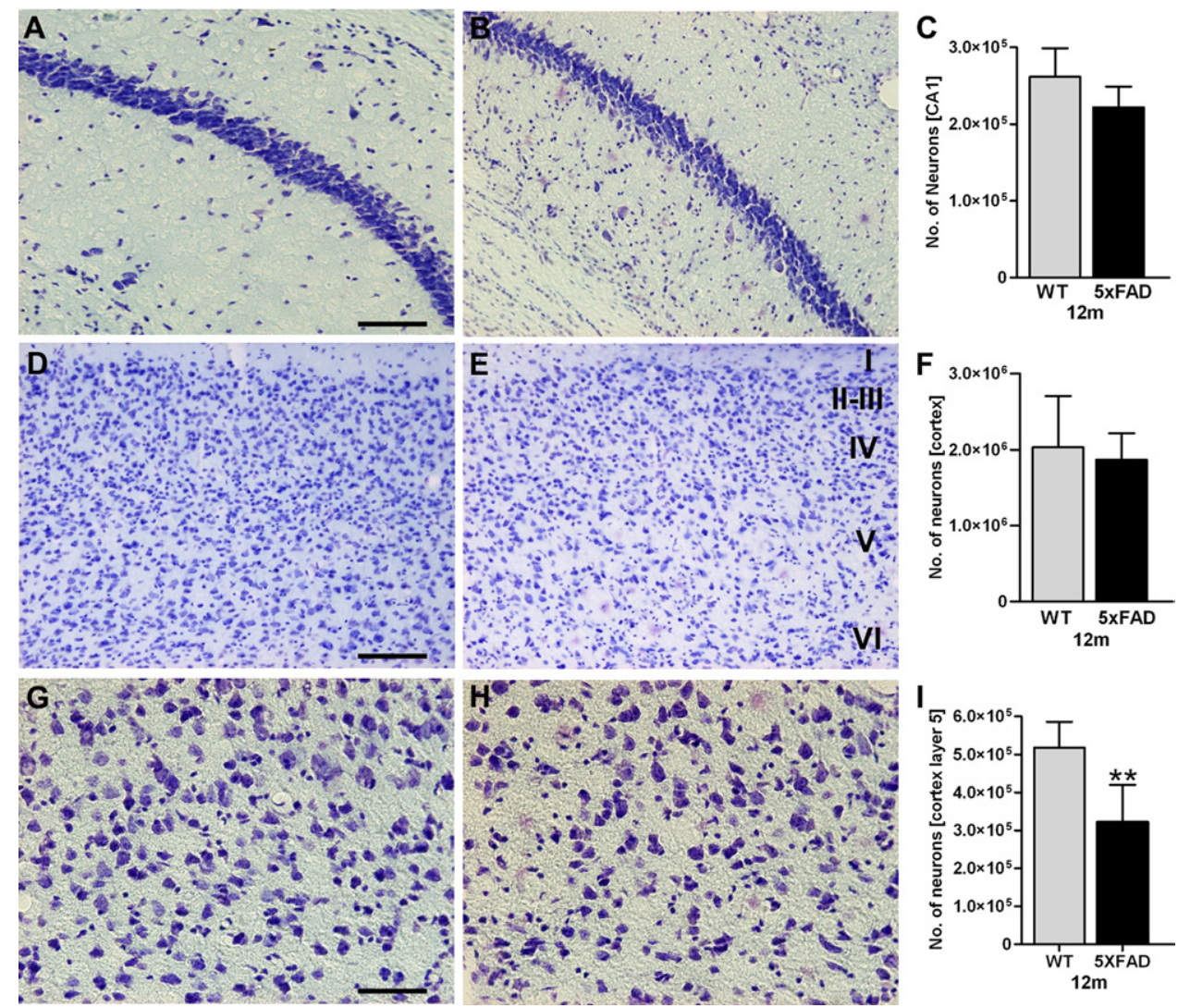

Fig. 4. Quantification of neurons in the CA1 (A-C), the overall frontal cortex (D-F) and the fifth cortical layer (G-I) of 5XFAD (B,E,H) and WT (A,D,G) mice at 12 months of age. While no difference in the number of neurons in CA1 (C) or the frontal cortex was detected (F), a neuron loss of $\sim 38 \%$ was noted in the fifth cortical layer of 5XFAD mice compared with WT (I). All error bars represent s.e.m. ** $p<0.01$. Scale bars: (A,B,D,E) $200 \mu$ m; (D,H) $50 \mu \mathrm{m}$.

represented by deficits in motor performance and increased axonal spheroid formation.

The spatial working memory was analyzed to recapitulate if the previously published impairment in the Y-maze task (Oakley et al., 2006) is still detectable in mice kept on a different genetic background. In the present study, the cross-maze was used, which is based on the same principle as the Y-maze task. The only difference between the two mazes is that the cross-maze presents a higher level of complexity because it has four arms instead of the three arms in the Y-maze which makes it more sensitive to depict memory impairments. In agreement with previous results (Oakley et al., 2006), 5XFAD mice showed a reduced alternation percentage in the cross maze starting from 6 months of age. This reduction was obviously not due to decreased motor activity because the 5XFAD mice showed a comparable number of arm entries as their WT littermates. Reduced working memory levels that decline with age have been demonstrated in a variety of $\mathrm{AD}$ transgenic mouse models (Chapman et al., 1999; Duyckaerts et al., 2008; Lovasic et al., 2005; Wirths et al., 2008). 5XFAD mice have been previously demonstrated to exhibit deficits in memory consolidation, demonstrated by significantly lower levels of contextual freezing starting at the age of 5-6 mo (Devi and Ohno, 2010; Ohno et al., 2006; Ohno, 2009). In good agreement, age-dependent impairments of basal synaptic transmission in hippocampal slices of 5XFAD mice have been described. At 6 mo of age, significant reductions in synaptic transmission were detected, whereas 4 mo old 5XFAD mice showed no impairment (Kimura and Ohno, 2009).

In addition, 5XFAD mice demonstrated reduced anxiety by spending more time in the open arms of the elevated plus-maze in comparison with their WT littermates starting from an early age (6 mo old) which further increased in an age-dependent manner. Various AD mouse models showed different anxiety profiles. Mouse models like Tg2576, PS1A246E or bigenic APPswe + PS1/DeltaE9 and APP/PS1KI mice have shown reduced anxiety (Faure et al., 2009; Lalonde et al., 2003a; Lalonde et al., 2003b; Lalonde et al., 2005), whereas other AD mouse models, such as APP23 did not demonstrate any change in their anxiety level (Lalonde et al., 2002). Analysis of anxiety, exploration and locomotor activity in the open field paradigm confirmed the reduction in anxiety detected using elevated plus maze because the 5XFAD mice spent more time in the central region com- 

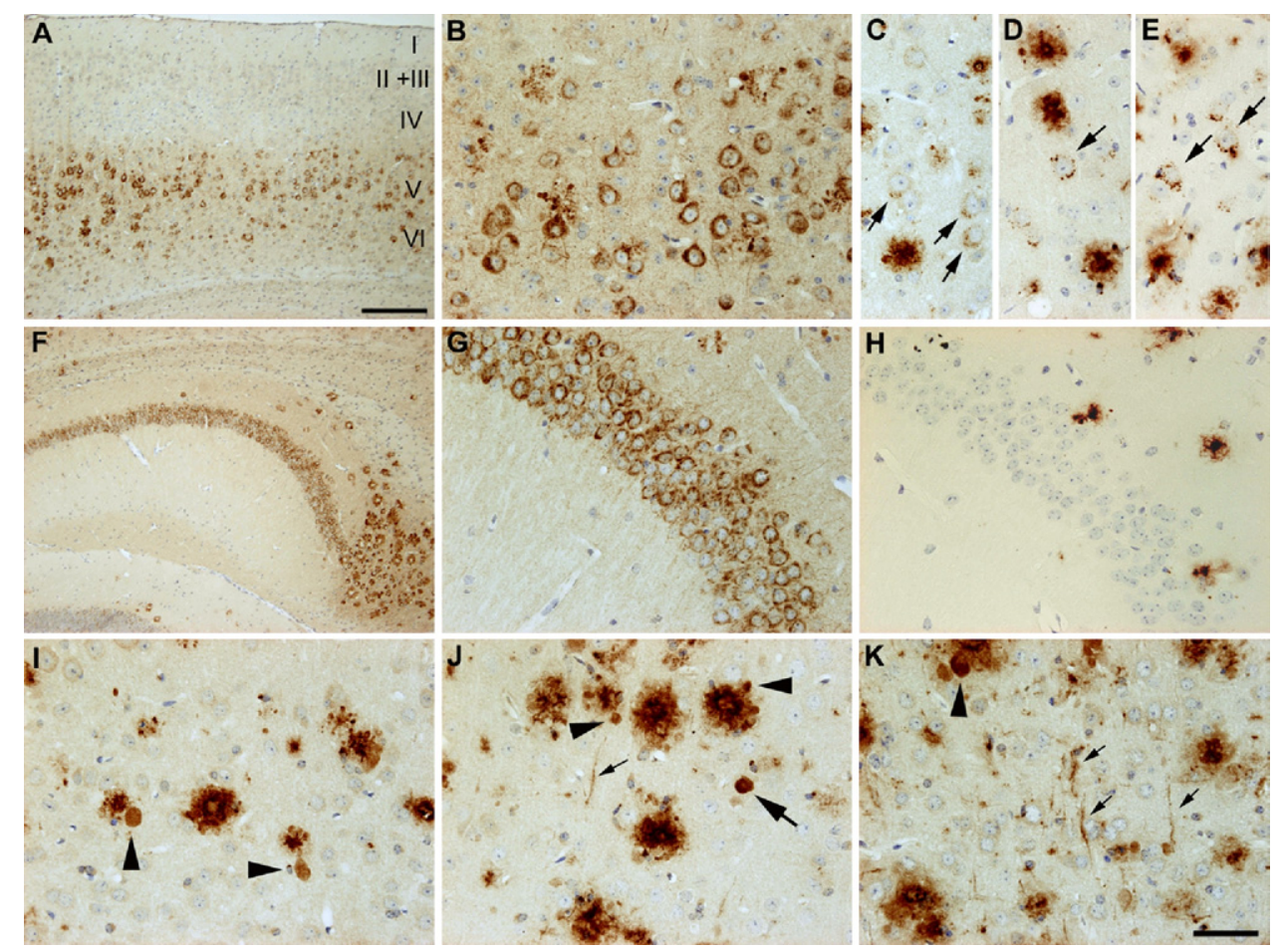

Fig. 5. Immunostaining for APP and intraneuronal $A \beta$ in the cortex (A-E,I-K) and CA1 (F-H). Staining against APP revealed comparable expression levels in the fifth cortical layer $(\mathrm{A}, \mathrm{B})$ and the CA1 region $(\mathrm{F}, \mathrm{G})$. However, staining with $\mathrm{G} 2-10(\mathrm{~A} \beta 40, \mathrm{C}), \mathrm{A} \beta[\mathrm{N}]\left(\mathrm{A} \beta_{1-\mathrm{x}}, \mathrm{D}\right), 692(\mathrm{~A} \beta, \mathrm{E})$ showed that the intraneuronal $\mathrm{A} \beta$ accumulation (arrows) was limited to the fifth cortical layer but not the CA1 pyramidal layer $(\mathrm{A} \beta[\mathrm{N}] ; \mathrm{H})$. A $\beta 40$ was detected in plaque-associated axonal spheroids (arrowhead) in 3- (I), 6- (J) and 12 month old 5XFAD mice. In addition plaque-independent A $\beta 40$-positive spheroids (large arrows) and A $\beta 40$-positive neuritic processes (small arrows) were detected. Scale bars: (A): $200 \mu \mathrm{m}$, (B-K) $50 \mu \mathrm{m}$.

pared with their WT littermates. In contrast, locomotor behavior did not seem to be affected because there was no difference detected in the speed or the traveled distance during the 5 min of testing.
Neuron loss in cortical layer 5 neurons has been previously observed in 5XFAD mice; however, no quantitative data on the extent was available (Oakley et al., 2006). Compared with age-matched WT mice, a significant neuron

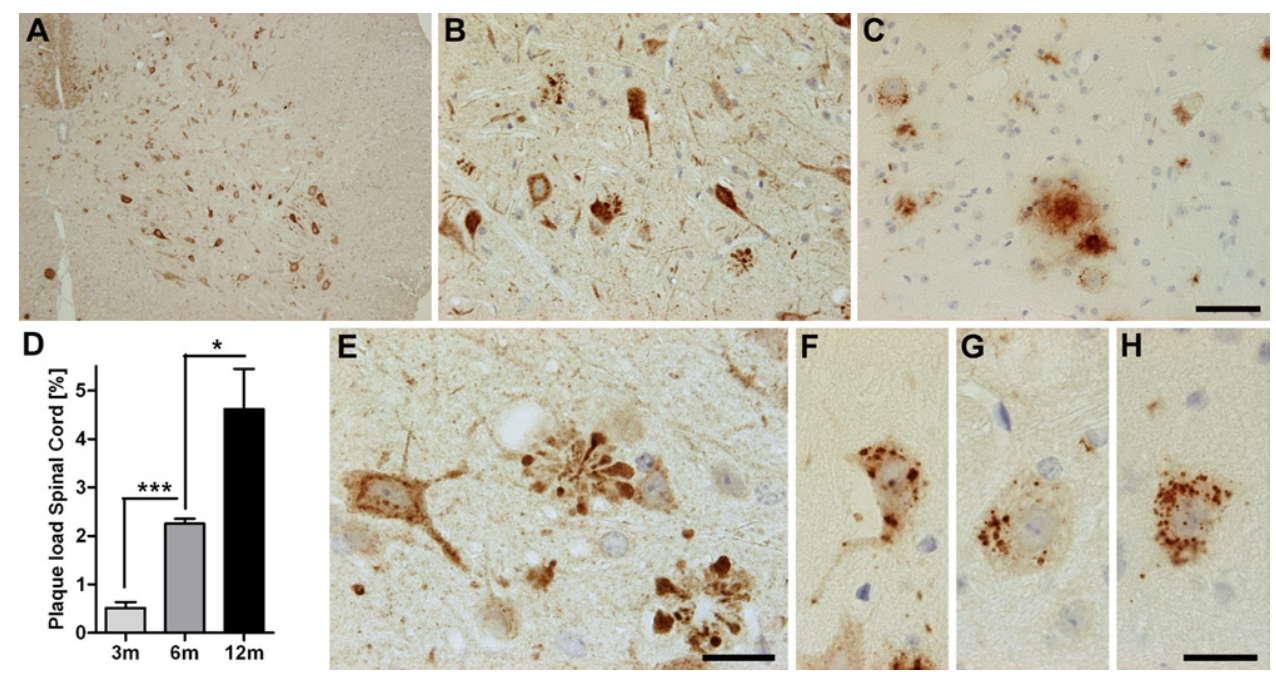

Fig. 6. APP and A $\beta$ staining in the spinal cord of 5XFAD mice. Staining against APP revealed expression in motor neurons of the spinal cord already at 3 months of age (A,B,E). 4G8 antibody showed plaque pathology and intraneuronal A $\beta$ as early as 3 months of age (C,F). Staining with other antibodies against $\mathrm{A} \beta$, such as $692(\mathrm{G})$ and $\mathrm{A} \beta[\mathrm{N}](\mathrm{H})$ confirmed the finding of intraneuronal $\mathrm{A} \beta$ in spinal cord motor neurons. In addition, quantification of the $\mathrm{A} \beta$ plaque load demonstrated a continuous increase with age (D). All error bars represent SEM *** $p<0.001$, * $p<0.05$. Scale bars: (A): $200 \mu$ m; (B,C) 50 $\mu \mathrm{m} ;(\mathrm{E}-\mathrm{H}) 20 \mu \mathrm{m}$. 

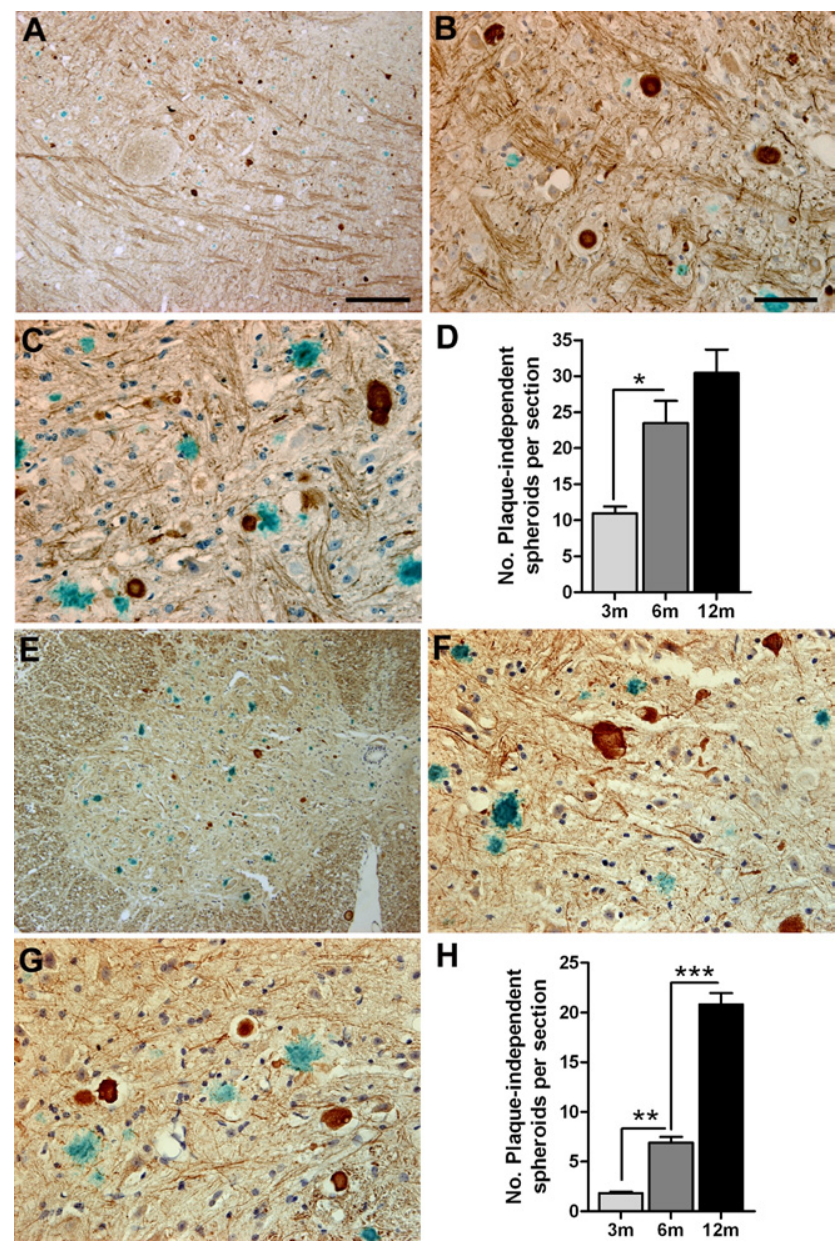

Fig. 7. Double immunostaining showing $\mathrm{A} \beta$ in blue using antibody $4 \mathrm{G} 8$ together with visualization of fibers in reddish-brown using NF-200 in the pons (Z-D) and the spinal cord (E-H) of 5XFAD mice. Axonal swelling are shown in 6- (A,B,E,F) and 12 month old $(\mathrm{C}, \mathrm{G})$ mice. Quantification of plaque-independent-axonal swellings revealed a significant increase from 3 to 6 months of age in the pons (D), while it showed a continuous agedependent increase in the spinal cord $(\mathrm{H})$. All error bars represent SEM $* * * p<0.001, * * p<0.01 ; * p<0.05$. Scale bars: (A,E) $200 \mu \mathrm{m}$; (B,C,F,G) $50 \mu \mathrm{m}$.

loss could be detected in layer 5 neurons of the cortex in 12 mo old 5XFAD mice in the present study. These neurons express human APP and accumulate considerable amounts of $\mathrm{A} \beta$ peptides already at the age of $3 \mathrm{mo}$. On the contrary, no neuron loss could be detected in the CA1 layer of the hippocampus which shows comparable levels of transgenic APP expression, however, without intraneuronal A $\beta$ accumulation. This confirms previous findings in APP/PS1KI mice, where neuron loss only occurs in brain regions showing intraneuronal $\mathrm{A} \beta$ accumulation, like frontal cortex, $\mathrm{CA} 1$ or distinct cholinergic brain stem nuclei. In the thalamus or the striatum, representing regions where only extracellular plaques are detectable, no neuron loss could be demonstrated (Breyhan et al., 2009; Casas et al., 2004; Christensen et al., 2008; Christensen et al., 2010), arguing against a significant role of extracellular plaques in terms of $\mathrm{A} \beta$ toxicity. In good agreement, only a weak correlation between plaque density and hippocampus-dependent memory has been found in 8 mo old 5XFAD mice (Kaczorowski et al., 2009). Very recently, a new APP transgenic mouse model has been described harboring the E693 $\Delta$ mutation. These mice do not show any extracellular amyloid deposits even at 24 mo of age, but reveal CA3 neuron loss at this time point which is preceded by intraneuronal $\mathrm{A} \beta$ oligomer accumulation (Tomiyama et al., 2010). Taken together, the recent data from APP/PS1KI, 5XFAD and APP E693A mice indicate that there is compelling evidence for a central role of intraneuronal $A \beta$ accumulation leading to neuron loss in APP transgenic mouse models of AD.

In addition to $A \beta$ peptides located in somatodendritic compartments, $A \beta$ was also detected in axonal spheroids and neuritic processes, adding further evidence to its intraneuronal localization. This corroborates previous data in APP/PS1KI mice (Wirths et al., 2007) and supports the idea that $\mathrm{A} \beta$ might be produced or released within axons (Kamal et al., 2001; Zhu et al., 2005). It has also been hypothesized that axonal swellings precede and participate in amyloid plaque formation, probably because of focally increased $A \beta$ secretion or lysis of swellings enriched in $\mathrm{A} \beta$ peptides (Stokin et al., 2005).

In conclusion, the 5XFAD mouse model recapitulates a variety of $\mathrm{AD}$ hallmarks, including working memory impairment, reduced anxiety corresponding to disinhibitory tendencies seen in $\mathrm{AD}$ patients, extensive extracellular plaque formation and selective neuron loss, making it a highly valuable research model. As neurofibrillary tangle formation is lacking, this model might represent a model for the "plaque predominant" type of $\mathrm{AD}$ which has been previously described in various autopsy series (Duyckaerts et al., 2009; Hansen et al., 1993; Tiraboschi et al., 2004). However, as intraneuronal $\mathrm{A} \beta$ plays a significant role in terms of the pathological alterations characterizing this model, it might be defined as an "amyloid-predominant" model of AD.

\section{Disclosure statement}

Animal studies were performed with the approval of the Local Research Ethics Committee in accordance with national and international guidelines. All efforts were made to minimize animal suffering and the number of animals used. The authors have no financial, personal, or other conflicts of interest to disclose.

\section{Acknowledgements}

The expert technical assistance of Petra Tucholla is gratefully acknowledged. We thank Gerd Multhaup for the generous gift of 23850 and 692 antibodies. This work was supported by The European Commission, Marie Curie Early Stage Training, MEST-CT-2005-020013 (NEURAD), Alz- 
heimer Ph.D. Graduate School, Alzheimer Forschung Initiative e.V. (to O.W.), and the Competence Network Degenerative Dementias of the German Federal Ministry of Education (grant no. 01 GI 0718, to T.A.B.).

\section{References}

Aoki, M., Volkmann, I., Tjernberg, L.O., Winblad, B., Bogdanovic, N., 2008. Amyloid beta-peptide levels in laser capture microdissected cornu ammonis 1 pyramidal neurons of Alzheimer's brain. Neuroreport 19, 1085-1089.

Arendash, G.W., Gordon, M.N., Diamond, D.M., Austin, L.A., Hatcher, J.M., Jantzen, P., DiCarlo, G., Wilcock, D., Morgan, D., 2001. Behavioral assessment of Alzheimer's transgenic mice following long-term Abeta vaccination: task specificity and correlations between Abeta deposition and spatial memory. DNA Cell Biol. 20, 737-744.

Breyhan, H., Wirths, O., Duan, K., Marcello, A., Rettig, J., Bayer, T.A., 2009. APP/PS1KI bigenic mice develop early synaptic deficits and hippocampus atrophy. Acta Neuropathol. 117, 677-685.

Calingasan, N.Y., Chen, J., Kiaei, M., Beal, M.F., 2005. beta-amyloid 42 accumulation in the lumbar spinal cord motor neurons of amyotrophic lateral sclerosis patients. Neurobiol. Dis. 19, 340-347.

Casas, C., Sergeant, N., Itier, J.M., Blanchard, V., Wirths, O., van der Kolk, N., Vingtdeux, V., van de Steeg, E., Ret, G., Canton, T., Drobecq, H., Clark, A., Bonici, B., Delacourte, A., Benavides, J., Schmitz, C., Tremp, G., Bayer, T.A., Benoit, P., Pradier, L., 2004. Massive p. CA1/2 neuronal loss with intraneuronal and $\mathrm{N}$-terminal truncated Abeta42 accumulation in a novel Alzheimer transgenic model. Am. J. Pathol. 165(4), 1289-1300.

Chapman, P.F., White, G.L., Jones, M.W., Cooper-Blacketer, D., Marshall, V.J., Irizarry, M., Younkin, L., Good, M.A., Bliss, T.V., Hyman, B.T., Younkin, S.G., Hsiao, K.K., 1999. Impaired synaptic plasticity and learning in aged amyloid precursor protein transgenic mice. Nat. Neurosci. 2, 271-276.

Chevalier-Larsen, E., Holzbaur, E.L., 2006. Axonal transport and neurodegenerative disease. Biochim. Biophys. Acta 1762, 1094-1108.

Christensen, D.Z., Bayer, T.A., Wirths, O., 2010. Intracellular Abeta triggers neuron loss in the cholinergic system of the APP/PS1KI mouse model of Alzheimer's disease. Neurobiol. Aging 31, 1153-1163.

Christensen, D.Z., Kraus, S.L., Flohr, A., Cotel, M.C., Wirths, O., Bayer, T.A., 2008. Transient intraneuronal Abeta rather than extracellular plaque pathology correlates with neuron loss in the frontal cortex of APP/PS1KI mice. Acta Neuropathol. 116, 647-655.

Devi, L., Ohno, M., 2010. Genetic reductions of beta-site amyloid precursor protein-cleaving enzyme 1 and amyloid-beta ameliorate impairment of conditioned taste aversion memory in 5XFAD Alzheimer's disease model mice. Eur. J. Neurosci. 31, 110-118.

Duyckaerts, C., Delatour, B., Potier, M.C., 2009. Classification and basic pathology of Alzheimer disease. Acta Neuropathol. 118, 5-36.

Duyckaerts, C., Potier, M.C., Delatour, B., 2008. Alzheimer disease models and human neuropathology: similarities and differences. Acta Neuropathol. 115, 5-38.

Ewers, M., Morgan, D.G., Gordon, M.N., Woodruff-Pak, D.S., 2006 Associative and motor learning in 12 month old transgenic APP +PS1 mice. Neurobiol. Aging 27, 1118-1128.

Faure, A., Verret, L., Bozon, B., El Tannir El Tayara, N., Ly, M., Kober, F., Dhenain, M., Rampon, C., Delatour, B., 2009. Impaired neurogenesis, neuronal loss, and brain functional deficits in the APPxPS1-Ki mouse model of Alzheimer's disease. Neurobiol. Aging Apr 22. [Epub ahead of print].

Gouras, G.K., Tsai, J., Naslund, J., Vincent, B., Edgar, M., Checler, F., Greenfield, J.P., Haroutunian, V., Buxbaum, J.D., Xu, H., Greengard, P., Relkin, N.R., 2000. Intraneuronal Abeta42 accumulation in human brain. Am. J. Pathol. 156, 15-20.
Gyure, K.A., Durham, R., Stewart, W.F., Smialek, J.E., Troncoso, J.C., 2001. Intraneuronal abeta-amyloid precedes development of amyloid plaques in Down syndrome. Arch. Pathol. Lab. Med. 125, 489-492.

Hansen, L.A., Masliah, E., Galasko, D., Terry, R.D., 1993. Plaque-only Alzheimer disease is usually the lewy body variant, and vice versa. J. Neuropathol. Exp. Neurol. 52, 648-654.

He, W., Barrow, C.J., 1999. The A beta 3-pyroglutamyl and 11-pyroglutamyl peptides found in senile plaque have greater beta-sheet forming and aggregation propensities in vitro than full-length A beta. Biochemistry $38,10871-1087$.

Kaczorowski, C.C., Sametsky, E., Shah, S., Vassar, R., Disterhoft, J.F., 2009. Mechanisms underlying basal and learning-related intrinsic excitability in a mouse model of Alzheimer's disease. Neurobiol. Aging Oct 13. [Epub ahead of print].

Kamal, A., Almenar-Queralt, A., LeBlanc, J.F., Roberts, E.A., Goldstein, L.S., 2001. Kinesin-mediated axonal transport of a membrane compartment containing beta-secretase and presenilin-1 requires APP. Nature 414, 643-648.

Karl, T., Pabst, R., von Horsten, S., 2003. Behavioral phenotyping of mice in pharmacological and toxicological research. Exp. Toxicol. Pathol. $55,69-83$.

Kimura, R., Ohno, M., 2009. Impairments in remote memory stabilization precede hippocampal synaptic and cognitive failures in 5XFAD Alzheimer mouse model. Neurobiol. Dis. 33, 229-235.

Knobloch, M., Konietzko, U., Krebs, D.C., Nitsch, R.M., 2007. Intracellular Abeta and cognitive deficits precede beta-amyloid deposition in transgenic arcAbeta mice. Neurobiol. Aging, 28, 1297-1306.

Kuo, Y.M., Emmerling, M.R., Woods, A.S., Cotter, R.J., Roher, A.E., 1997. Isolation, chemical characterization, and quantitation of A beta 3-pyroglutamyl peptide from neuritic plaques and vascular amyloid deposits. Biochem. Biophys. Res. Commun. 237, 188-191.

Lalonde, R., Dumont, M., Staufenbiel, M., Sturchler-Pierrat, C., Strazielle, C., 2002. Spatial learning, exploration, anxiety, and motor coordination in female APP23 transgenic mice with the Swedish mutation. Brain Res. 956, 36-44.

Lalonde, R., Kim, H.D., Maxwell, J.A., Fukuchi, K., 2005. Exploratory activity and spatial learning in 12 month old APP(695)SWE/co+PS1/ DeltaE9 mice with amyloid plaques. Neurosci Lett 390(2), 87-92.

Lalonde, R., Lewis, T.L., Strazielle, C., Kim, H., Fukuchi, K., 2003 a. Transgenic mice expressing the betaAPP695SWE mutation: effects on exploratory activity, anxiety, and motor coordination. Brain Res. 977, $38-45$.

Lalonde, R., Qian, S., Strazielle, C., 2003b. Transgenic mice expressing the PS1-A246E mutation: effects on spatial learning, exploration, anxiety, and motor coordination. Behav. Brain Res. 138, 71-79.

Le Cudennec, C., Faure, A., Ly, M., Delatour, B., 2008. One-year longitudinal evaluation of sensorimotor functions in APP751SL transgenic mice. Genes Brain Behav. 7 suppl 1, 83-91.

Lee, K.W., Lee, S.H., Kim, H., Song, J.S., Yang, S.D., Paik, S.G., Han, P.L., 2004. Progressive cognitive impairment and anxiety induction in the absence of plaque deposition in C57BL/6 inbred mice expressing transgenic amyloid precursor protein. J. Neurosci. Res. 76, 572-580.

Lord, A., Kalimo, H., Eckman, C., Zhang, X.Q., Lannfelt, L., Nilsson, L.N., 2006. The Arctic Alzheimer mutation facilitates early intraneuronal Abeta aggregation and senile plaque formation in transgenic mice. Neurobiol. Aging 27, 67-77.

Lovasic, L., Bauschke, H., Janus, C., 2005. Working memory impairment in a transgenic amyloid precursor protein TgCRND8 mouse model of Alzheimer's disease. Genes Brain Behav. 4, 197-208.

O'Keeffe, S.T., Kazeem, H., Philpott, R.M., Playfer, J.R., Gosney, M., Lye, M., 1996. Gait disturbance in Alzheimer's disease: a clinical study. Age Ageing 25, 313-316.

Oakley, H., Cole, S.L., Logan, S., Maus, E., Shao, P., Craft, J., GuillozetBongaarts, A., Ohno, M., Disterhoft, J., Van Eldik, L., Berry, R., Vassar, R., 2006. Intraneuronal beta-amyloid aggregates, neurodegeneration, and neuron loss in transgenic mice with five familial Alzhei- 
mer's disease mutations: potential factors in amyloid plaque formation. J. Neurosci. 26, 10129-10140.

Oddo, S., Caccamo, A., Shepherd, J.D., Murphy, M.P., Golde, T.E., Kayed, R., Metherate, R., Mattson, M.P., Akbari, Y., LaFerla, F.M., 2003. Triple-transgenic model of Alzheimer's disease with plaques and tangles: intracellular Abeta and synaptic dysfunction. Neuron 39, 409421.

Ohno, M., 2009. Failures to reconsolidate memory in a mouse model of Alzheimer's disease. Neurobiol. Learn. Mem. 92, 455-459.

Ohno, M., Chang, L., Tseng, W., Oakley, H., Citron, M., Klein, W.L., Vassar, R., Disterhoft, J.F., 2006. Temporal memory deficits in Alzheimer's mouse models: rescue by genetic deletion of BACE1. Eur. J. Neurosci. 23, 251-260.

Ohno, M., Cole, S.L., Yasvoina, M., Zhao, J., Citron, M., Berry, R., Disterhoft, J.F., Vassar, R., 2007. BACE1 gene deletion prevents neuron loss and memory deficits in 5XFAD APP/PS1 transgenic mice. Neurobiol. Dis. 26, 134-145.

Paxinos, G., Franklin, K.B.J., 2001. The Mouse Brain in Stereotaxic Coordinates, 2nd Ed. Academic Press.

Pettersson, A.F., Olsson, E., Wahlund, L.O., 2005. Motor function in subjects with mild cognitive impairment and early Alzheimer's disease. Dement. Geriatr. Cogn. Disord. 19, 299-304.

Probst, A., Gotz, J., Wiederhold, K.H., Tolnay, M., Mistl, C., Jaton, A.L., Hong, M., Ishihara, T., Lee, V.M., Trojanowski, J.Q., Jakes, R., Crowther, R.A., Spillantini, M.G., Burki, K., Goedert, M., 2000. Axonopathy and amyotrophy in mice transgenic for human four-repeat tau protein. Acta Neuropathol. 99, 469-481.

Russo, C., Violani, E., Saxlis, S., Venezia, V., Dolcini, V., Damonte, G., Benatti, U., D’Arrigo, C., Patrone, E., Carlo, P., Schettini, G., 2002. Pyroglutamate-modified amyloid beta-peptides-AbetaN3(pE) — strongly affect cultured neuron and astrocyte survival. J. Neurochem. 82, $1480-1489$.

Scarmeas, N., Albert, M., Brandt, J., Blacker, D., Hadjigeorgiou, G., Papadimitriou, A., Dubois, B., Sarazin, M., Wegesin, D., Marder, K., Bell, K., Honig, L., Stern, Y., 2005. Motor signs predict poor outcomes in Alzheimer disease. Neurology 64, 1696-1703.

Scarmeas, N., Hadjigeorgiou, G.M., Papadimitriou, A., Dubois, B., Sarazin, M., Brandt, J., Albert, M., Marder, K., Bell, K., Honig, L.S., Wegesin, D., Stern, Y., 2004. Motor signs during the course of Alzheimer disease. Neurology 63, 975-982.

Schilling, S., Lauber, T., Schaupp, M., Manhart, S., Scheel, E., Bohm, G., Demuth, H.U., 2006. On the seeding and oligomerization of pGluamyloid peptides (in vitro). Biochemistry 45, 12393-12399.

Schmitz, C., Hof, P.R., 2005. Design-based stereology in neuroscience. Neuroscience 130, 813-831.

Schmitz, C., Rutten, B.P., Pielen, A., Schafer, S., Wirths, O., Tremp, G., Czech, C., Blanchard, V., Multhaup, G., Rezaie, P., Korr, H., Steinbusch, H.W., Pradier, L., Bayer, T.A., 2004. Hippocampal neuron loss exceeds amyloid plaque load in a transgenic mouse model of Alzheimer's disease. Am. J. Pathol. 164, 1495-1502.

Spittaels, K., Van den Haute, C., Van Dorpe, J., Bruynseels, K., Vandezande, K., Laenen, I., Geerts, H., Mercken, M., Sciot, R., Van Lommel, A., Loos, R., Van Leuven, F., 1999. Prominent axonopathy in the brain and spinal cord of transgenic mice overexpressing four-repeat human tau protein. Am. J. Pathol. 155, 2153-2165.

Stokin, G.B., Lillo, C., Falzone, T.L., Brusch, R.G., Rockenstein, E., Mount, S.L., Raman, R., Davies, P., Masliah, E., Williams, D.S., Goldstein, L.S., 2005. Axonopathy and transport deficits early in the pathogenesis of Alzheimer's disease. Science 307, 1282-1288.

Takahashi, R.H., Milner, T.A., Li, F., Nam, E.E., Edgar, M.A., Yamaguchi, H., Beal, M.F., Xu, H., Greengard, P., Gouras, G.K., 2002. Intraneu- ronal Alzheimer abeta42 accumulates in multivesicular bodies and is associated with synaptic pathology. Am. J. Pathol. 161, 1869-1879.

Tesseur, I., Van Dorpe, J., Bruynseels, K., Bronfman, F., Sciot, R., Van Lommel, A., Van Leuven, F., 2000. Prominent axonopathy and disruption of axonal transport in transgenic mice expressing human apolipoprotein E4 in neurons of brain and spinal cord. Am. J. Pathol. 157, $1495-1510$.

Tiraboschi, P., Sabbagh, M.N., Hansen, L.A., Salmon, D.P., Merdes, A., Gamst, A., Masliah, E., Alford, M., Thal, L.J., Corey-Bloom, J., 2004. Alzheimer disease without neocortical neurofibrillary tangles: "a second look". Neurology 62, 1141-1147.

Tomiyama, T., Matsuyama, S., Iso, H., Umeda, T., Takuma, H., Ohnishi, K., Ishibashi, K., Teraoka, R., Sakama, N., Yamashita, T., Nishitsuji, K., Ito, K., Shimada, H., Lambert, M.P., Klein, W.L., Mori, H., 2010. A mouse model of amyloid \{beta\} oligomers: their contribution to synaptic alteration, abnormal tau phosphorylation, glial activation, and neuronal loss in vivo. J. Neurosci. 30, 4845-4856.

Van Broeck, B., Vanhoutte, G., Pirici, D., Van Dam, D., Wils, H., Cuijt, I., Vennekens, K., Zabielski, M., Michalik, A., Theuns, J., De Deyn, P.P., Van der Linden, A., Van Broeckhoven, C., Kumar-Singh, S., 2008. Intraneuronal amyloid beta and reduced brain volume in a novel APP T714I mouse model for Alzheimer's disease. Neurobiol. Aging 29, 241-252.

West, M.J., 2002. Design-based stereological methods for counting neurons. Prog. Brain Res. 135, 43-51.

West, M.J., Slomianka, L., Gundersen, H.J., 1991. Unbiased stereological estimation of the total number of neurons in the subdivisions of the rat hippocampus using the optical fractionator. Anat. Rec. 231, 482-497.

Wirths, O., Bayer, T.A., 2008. Motor impairment in Alzheimer's disease and transgenic Alzheimer's disease mouse models. Genes Brain Behav. 7 suppl 1, 1-5.

Wirths, O., Bethge, T., Marcello, A., Harmeier, A., Jawhar, S., Lucassen, P.J., Multhaup, G., Brody, D.L., Esparza, T., Ingelsson, M., Kalimo, H., Lannfelt, L., Bayer, T.A., 2010. Pyroglutamate Abeta pathology in APP/PS1KI mice, sporadic and familial Alzheimer's disease cases. J. Neural Transm. 117, 85-96.

Wirths, O., Breyhan, H., Cynis, H., Schilling, S., Demuth, H.U., Bayer, T.A., 2009. Intraneuronal pyroglutamate-Abeta 3-42 triggers neurodegeneration and lethal neurological deficits in a transgenic mouse model. Acta Neuropathol. 118, 487-496.

Wirths, O., Breyhan, H., Schafer, S., Roth, C., Bayer, T.A., 2008. Deficits in working memory and motor performance in the APP/PS1ki mouse model for Alzheimer's disease. Neurobiol. Aging 29, 891-901.

Wirths, O., Multhaup, G., Czech, C., Blanchard, V., Moussaoui, S., Tremp, G., Pradier, L., Beyreuther, K., Bayer, T.A., 2001. Intraneuronal Abeta accumulation precedes plaque formation in beta-amyloid precursor protein and presenilin-1 double-transgenic mice. Neurosci. Lett. 306, $116-120$.

Wirths, O., Multhaup, G., Czech, C., Feldmann, N., Blanchard, V., Tremp, G., Beyreuther, K., Pradier, L., Bayer, T.A., 2002. Intraneuronal APP/A beta trafficking and plaque formation in beta-amyloid precursor protein and presenilin-1 transgenic mice. Brain Pathol. 12, 275-286.

Wirths, O., Weis, J., Kayed, R., Saido, T.C., Bayer, T.A., 2007. Agedependent axonal degeneration in an Alzheimer mouse model. Neurobiol. Aging 28, 1689-1699.

Wirths, O., Weis, J., Szczygielski, J., Multhaup, G., Bayer, T.A., 2006. Axonopathy in an APP/PS1 transgenic mouse model of Alzheimer's disease. Acta Neuropathol. 111, 312-319.

Zhu, X., Moreira, P.I., Smith, M.A., Perry, G., 2005. Alzheimer's disease: an intracellular movement disorder? Trends Mol. Med. 11, 391-393. 


\section{Project II: "Evaluating the role of glutaminyl cyclase and pyroglutamate-modified $A \beta$ in AD via the 5XFAD/hQC and 5XFAD/QC- KO mouse models"}

\subsection{Overview}

$\mathrm{A} \beta_{\mathrm{pE3}-\mathrm{x}}$ represents a major $\mathrm{A} \beta$ fraction present in $\mathrm{AD}$ patients that is receiving a considerable attention due to its higher aggregation propensity, stability and increased toxicity compared with full-length $A \beta$ (Gunn et al., 2010). It has been demonstrated that $Q C$ is involved in the formation of $\mathrm{A} \beta_{\mathrm{PE}-\mathrm{x}}$ (Schilling et al., 2004; Cynis et al., 2006; Cynis et al., 2008a; Gunn et al., 2010).

Increased QC RNA and protein expression was identified in the cortex of AD patients in comparison to non-demented aged controls. This increase in QC was claimed to parallel higher $A \beta_{\mathrm{pE} 3-42}$ levels in $\mathrm{AD}$. Oral administration of a $\mathrm{QC}$ inhibitor to Tg2576 and TASD-41 mice reduced $A \beta_{\mathrm{pE3}-\mathrm{x}}, \mathrm{A} \beta_{\mathrm{x}-40}$ and $\mathrm{A} \beta_{\mathrm{x}-42}$ levels. This reduction was accompanied by a decrease in plaque density and gliosis in addition to improvements in contextual fear memory and spatial memory (Schilling et al., 2008c). However, both models harbor low $\mathrm{A} \beta_{\mathrm{pE} 3-\mathrm{x}}$ levels and milder AD pathology in comparison to the 5XFAD model (Hsiao et al., 1996; Rockenstein et al., 2001).

In order to study the effect of ectopic QC overexpression on overall AD pathology, 5XFAD mice were crossed with transgenic mice expressing human $\mathrm{QC}(\mathrm{hQC})$ under the control of the neuron-specific Thy-1 promoter. hQC mice have no obvious phenotype, normal life span and breeding performance indicating no severe side effects of the transgene integration.

Moreover, the effect of endogenous QC was studied by generating 5XFAD/QC-KO mice (homozygous QC knock-out). The consequences of genetic QC ablation, on endocrine function and behavior have been very recently described. Total QC ablation did not show effects on fertility, weight or behavior as QC-KO mice performed indistinguishably from wild type mice in motor tasks, fear conditioning task, general activity and ingestion behavior (Schilling et al., 2011). 
The changes in QC levels in the different genotypes were verified at tissue and RNA levels. The effect of $\mathrm{QC}$ on $\mathrm{A} \beta$ and plaque pathology was investigated. Besides, behavioral comparison of all the genotypes at different behavioral entities was conducted at 6 months of age.

\section{Aims of project II}

- To elucidate in vivo the role played by QC in AD using an aggressive AD mouse model.

- To understand the effect of ectopic QC overexpression on $\mathrm{A} \beta_{\mathrm{pE} 3-\mathrm{x}}$ level in the 5XFAD mouse model.

- To unravel the effects of $A \beta_{\mathrm{pE} 3-\mathrm{x}}$ increase on the severity of the $\mathrm{AD}$ behavioral phenotype in the 5XFAD mice.

- To evaluate the impact of abolishing endogenous $\mathrm{QC}$ on $\mathrm{A} \beta_{\mathrm{pE} 3-\mathrm{x}}$ level in the 5XFAD mouse model.

- To determine whether QC-KO can reduce the plaque pathology and rescue the behavioral deficits seen in the 5XFAD mouse model. 


\subsection{Original publication}

Jawhar, S.*, Wirths, O.*, Schilling, S.*, Graubner, S., Demuth, H.U., and Bayer, T.A. (2011). Overexpression of glutaminyl cyclase, the enzyme responsible for pyroglutamate Abeta formation, induces behavioral deficits, and glutaminyl cyclase knock-out rescues the behavioral phenotype in 5XFAD mice. The Journal of Biological Chemistry. 286: 44544460 .

* Shared co-authorship

\section{Personal contribution}

I was involved in the design of the study. Also, I conducted all the behavioral and immunohistochemical analysis, western blot and RT-PCR experiments. I was responsible for the analysis and interpretation of the behavior, plaque load and ELISA data. I made substantial contributions to the writing and organization of the manuscript. Dr. Oliver Wirths was responsible for arranging the mice breeding. Dr. Stefan Schilling from Probiodrug AG provided the transgenic QC mice and the QC-KO mice.

\section{Contribution to figures}

I fully contributed to figures $1,2,3,5$, supplementary figure 1 and supplementary figure 2 . In figure 4, ELISA was conducted by Probiodrug AG, however, I performed the statistical analysis, the interpretation of the results and the preparation of the figure. Supplementary figure 3 was performed by Probiodrug AG. 


\section{Overexpression of Glutaminyl Cyclase, the Enzyme Responsible for Pyroglutamate A $\beta$ Formation, Induces Behavioral Deficits, and Glutaminyl Cyclase Knock-out Rescues the Behavioral Phenotype in 5XFAD Mice ${ }^{*[s]}$}

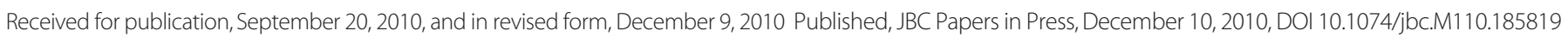
Sadim Jawhar ${ }^{\ddagger 1}$, Oliver Wirths ${ }^{\ddagger 1}$, Stephan Schilling ${ }^{\S 1}$, Sigrid Graubner ${ }^{\natural}$, Hans-Ulrich Demuth ${ }^{\S 2}$, and Thomas A. Bayer ${ }^{\ddagger 3}$

From the ${ }^{\ddagger}$ Department of Molecular Psychiatry and Alzheimer Ph.D. Graduate School, University Medicine Goettingen, 37075 Goettingen, Germany, §Probiodrug AG, 06120 Halle, Germany, and "Ingenium Pharmaceuticals GmbH, 82152 Munich, Germany

Pyroglutamate-modified A $\beta(\mathrm{A} \beta \mathrm{pE} 3-42)$ peptides are gaining considerable attention as potential key players in the pathology of Alzheimer disease (AD) due to their abundance in AD brain, high aggregation propensity, stability, and cellular toxicity. Overexpressing $\mathrm{A} \beta \mathrm{pE} 3-42$ induced a severe neuron loss and neurological phenotype in TBA2 mice. In vitro and in vivo experiments have recently proven that the enzyme glutaminyl cyclase $(\mathrm{QC})$ catalyzes the formation of $\mathrm{A} \beta \mathrm{pE} 3-42$. The aim of the present work was to analyze the role of $\mathrm{QC}$ in an $\mathrm{AD}$ mouse model with abundant $\mathrm{A} \beta \mathrm{pE} 3-42$ formation. 5XFAD mice were crossed with transgenic mice expressing human QC (hQC) under the control of the Thy1 promoter. 5XFAD/hQC bigenic mice showed significant elevation in TBS, SDS, and formic acid-soluble $\mathrm{A} \beta \mathrm{pE} 3-42$ peptides and aggregation in plaques. In 6-month-old 5XFAD/hQC mice, a significant motor and working memory impairment developed compared with 5XFAD. The contribution of endogenous QC was studied by generating 5XFAD/QC-KO mice (mouse QC knock-out). 5XFAD/QC-KO mice showed a significant rescue of the wild-type mice behavioral phenotype, demonstrating the important contribution of endogenous mouse QC and transgenic overexpressed QC. These data clearly demonstrate that $\mathrm{QC}$ is crucial for modulating $\mathrm{A} \beta \mathrm{pE} 3-42$ levels $i n$ vivo and prove on a genetic base the concept that reduction of $\mathrm{QC}$ activity is a promising new therapeutic approach for AD.

Alzheimer disease $(\mathrm{AD})^{4}$ is a progressive neurodegenerative disorder characterized by the presence of extracellular amyloid plaques composed of amyloid- $\beta(\mathrm{A} \beta)$ and intracellular

* This work was supported by the German Federal Department of Education, Science and Technology, Grant 3013185 to a collaborative consortium led by H.-U. D.'s group, including T. A. B.'s team.

5 The on-line version of this article (available at http://www.jbc.org) contains supplemental Methods and Figs. 1-3.

${ }^{1}$ These authors contributed equally to this work.

${ }^{2}$ To whom correspondence may be addressed: Probiodrug AG, Weinbergweg 22, 06120 Halle (Saale), Germany. E-mail: Hans-Ulrich.Demuth@ probiodrug.de.

${ }^{3}$ To whom correspondence may be addressed: Division of Molecular Psychiatry, University Medicine Göttingen, Von-Siebold-Strasse 5, 37075 Göttingen, Germany. E-mail: tbayer@gwdg.de.

${ }^{4}$ The abbreviations used are: $A D$, Alzheimer disease; $A \beta$, amyloid- $\beta ; A \beta p E 3$, pyroglutamate $A \beta$; APP, amyloid precursor protein; QC, glutaminyl cyclase; hQC, human QC; QC-KO, QC knock-out. neurofibrillary tangles. The discovery that certain early onset familial forms of $\mathrm{AD}$ may be caused by enhanced levels of $\mathrm{A} \beta$ peptides has led to the hypothesis that amyloidogenic $\mathrm{A} \beta$ is intimately involved in the pathogenic process (1).

Besides full-length $A \beta 40$ and 42 isoforms starting with an aspartate at position 1 , a variety of different $\mathrm{N}$-truncated $\mathrm{A} \beta$ peptides have been identified in AD brains. Ragged peptides including phenylalanine at position 4 of $\mathrm{A} \beta$ have been reported as early as 1985 by Masters et al. (2). In contrast, no $\mathrm{N}$-terminal sequence could be obtained from cores purified in a SDS-containing buffer, which led to the assumption that the $\mathrm{N}$ terminus could be blocked $(3,4)$.

The presence of $\mathrm{A} \beta \mathrm{pE} 3(\mathrm{~N}$-terminally truncated $\mathrm{A} \beta$ starting with pyroglutamate) in AD brain was subsequently shown using mass spectrometry of purified $\mathrm{A} \beta$ peptides, explaining at least partially initial difficulties in sequencing $\mathrm{A} \beta$ peptides purified from human brain tissue (5). The authors reported that only $10-15 \%$ of the total $A \beta$ isolated by this method begins at position 3 with A $\beta \mathrm{pE} 3$. Saido et al. (6) and others (7) subsequently showed that $\mathrm{A} \beta \mathrm{pE} 3$ represents a dominant fraction of $\mathrm{A} \beta$ peptides in $\mathrm{AD}$ brain.

Overexpression of $\mathrm{A} \beta \mathrm{pE} 3-42$ in neurons of TBA2 transgenic mice triggers neuron loss and an associated neurological phenotype (8). N-terminal $\mathrm{pE}$ formation can be catalyzed by glutaminyl cyclase $(\mathrm{QC})$ and is pharmacologically inhibited by QC inhibitors, both in vitro (9) and in vivo (10). Moreover, QC expression was found up-regulated in the cortex of patients with $\mathrm{AD}$ and correlated with the appearance of $\mathrm{pE}$ modified A $\beta$. Oral application of a QC inhibitor resulted in reduced $\mathrm{A} \beta \mathrm{pE} 3-42$ burden in two different transgenic mouse models of AD as well as in a transgenic Drosophila model. Interestingly, treatment of these mice was accompanied by reductions in $\mathrm{A} \beta \mathrm{x}-40 / 42$, diminished plaque formation and gliosis, as well as improved performance in context memory and spatial learning tests (10). Thus, $\mathrm{A} \beta \mathrm{pE} 3-42$ reduction is a promising target for therapy of AD. In the current work, the contribution of QC was studied for the first time using genetic means by human QC overexpression and endogenous QC-knock-out in an AD mouse model.

\section{EXPERIMENTAL PROCEDURES}

Transgenic and Knock-out Mice-5XFAD (11) mice have 51 
more than 10 generations on a C57BL/6J genetic background and housed at a 12-h day/12-h night cycle with free access to food and water. For generation of hQC transgenic mice, an expression vector containing the cDNA of human QC under control of the murine Thy1 promoter sequence was constructed, applying standard molecular biology techniques and verified by sequencing. The transgenic founder was generated on C57BL/6J/CBA background by pronuclear injection (JSW, Graz, Austria). The resulting offspring were further characterized for transgene integration by PCR analysis and after crossing to C57BL/6J wild-type mice for transgene expression by RT-PCR (more than 10 generations). QC knock-out mice (QC-KO) were generated on the basis of a classical homologous recombination approach at Genoway, Lyon. The targeting vector contained the mouse chromosomal QC region ranging from intron 3 to exon 6 . This region was modified by insertion of two LoxP sites in intron 3 and 5, respectively. In addition, a neomycin resistance cassette flanked by two flippase recognition targets was inserted immediately upstream of the LoxP in intron 5. After homologous recombination and chimera production, the neomycin selection cassette was removed by breeding with Flp-expressing mice followed by breeding of the pups with Cre-expressing mice for deletion of QC exons 4 and 5. The deletion of exons 4 and 5 causes a frameshift in the QC open reading frame generating a stop codon in exon 6. Successful manipulation was confirmed by PCR and Southern hybridization. Absence of murine QC in 5XFAD/QC-KO comparison with 5XFAD and 5XFAD/hQC was further confirmed by RT-PCR (supplemental Methods and supplemental Fig. 1). Animals were handled according to German guidelines for animal care and studies were approved by the local legal authorities (LAVES). Only female mice were used.

Immunohistochemistry-Mouse tissue was processed as described previously (12). In brief, 4- $\mu \mathrm{m}$ paraffin sections were pretreated with $0.3 \% \mathrm{H}_{2} \mathrm{O}_{2}$ in PBS to block endogenous peroxidases, and antigen retrieval was achieved by boiling sections in $0.01 \mathrm{M}$ citrate buffer, $\mathrm{pH}$ 6.0, followed by a 3-min incubation in $88 \%$ formic acid. Primary antibodies were incubated overnight, followed by incubation with biotinylated secondary antibodies (DAKO) before staining was visualized using the $\mathrm{ABC}$ method with Vectastain kit (Vector Laboratories) and diaminobenzidine as chromogen. Alternatively, fluorochromated secondary antibodies (anti-mouse Alexa Fluor 594 and anti-rabbit Alexa Fluor 488; Invitrogen) were used for immunofluorescence detection.

Antibodies-A $\beta$ antibodies NT78 (against generic A $\beta$; Synaptic Systems), 22C11 (APP; Millipore) and 2-48 (against N-terminal A $\beta p E 3$; Synaptic Systems) (12) were used. Antisera (against QC) were raised against recombinant full-length mouse QC (1301) and have been proven to recognize hQC (13).

ELISA of A $\beta$ Levels in Brain-Frozen brains $(n=4-8$ per group) were weighed and subsequently subjected to a sequential $A \beta$ extraction. In a first step, brains were homogenized in TBS (120 mm NaCl, $50 \mathrm{~mm}$ Tris, pH 8.0, containing complete protease inhibitor (Roche Applied Science)) using a Dounce homogenizer, sonified, and subsequently centrifuged at
$27,000 \times g$ for $20 \mathrm{~min}$ at $4{ }^{\circ} \mathrm{C}$. The supernatant was removed and stored at $-80{ }^{\circ} \mathrm{C}$. The pellet was dissolved in $2.5 \mathrm{ml}$ of $2 \%$ SDS, sonificated, and subsequently centrifuged at 80,000 $\times g$ for $1 \mathrm{~h}$ at $4{ }^{\circ} \mathrm{C}$. Supernatants were directly frozen at $-80^{\circ} \mathrm{C}$. The resulting pellets were again resuspended in $0.5 \mathrm{ml}$ of $70 \%$ formic acid, sonified, and neutralized using $1 \mathrm{M}$ Tris. Aliquots of the neutralized formic acid fraction were directly frozen at $-80{ }^{\circ} \mathrm{C}$. SDS lysates were diluted at least 10 -fold for determination of $\mathrm{A} \beta \mathrm{x}-42$ and $\mathrm{A} \beta \mathrm{pE} 3$ using ELISA. All dilutions were carried out using EIA buffer (IBL Co.). The neutralized formic acid fraction and the TBS fraction were applied directly or after dilution using EIA buffer. ELISA measurements were performed in triplicate and according to the protocol of the manufacturer (IBL Co.; catalog nos. JP27716 and JP27711). Samples were run in triplicate.

Quantification of Plaque Load-Extracellular A $\beta$ load was evaluated in mouse brain using an Olympus BX-51 microscope equipped with an Olympus DP-50 camera and the Image J software (V1.41; National Institutes of Health). Serial images of $40 \times$ magnification (hippocampus) and $100 \times$ (cortex) were captured on six sections/animal ( $n=5$ /group), which were at least $30 \mu \mathrm{m}$ afar from each other. Using ImageJ the pictures were binarized to 16-bit black and white images, and a fixed intensity threshold was applied defining the DAB staining.

Behavioral Testing-Spontaneous alternation rates were assessed using $\mathrm{Y}$ - and cross-maze as described previously (11, 14). The alternation percentage was calculated as the percentage of actual alternations to the total number of arm entries. Balance and general motor function were assessed using the balance beam task. A 1-cm dowel beam is attached to two support columns $44 \mathrm{~cm}$ above a padded surface. At either end of the 50 -cm long beam, a $9 \times 15$-cm escape platform is attached. The animal is placed on the center of the beam and released. Each animal is given three trials during a single day of testing. The time the animal remained on the beam is recorded and the resulting latencies to fall of all three trials are averaged. If an animal remains on the beam for whole 60-s trial or escapes to one of the platforms, the maximum time of $60 \mathrm{~s}$ is recorded (14). For the string suspension test the animals are permitted to grasp the string by their forepaws and are released. A rating system from 0 to 5 is used during the single 60-s trial to assess each animal's performance in this task: $0=$ unable to remain on the string; $1=$ hangs only by fore- or hindpaws; 2 = as for 1 , but attempts to climb onto string; $3=$ sits on string and is able to hold balance; $4=$ four paws and tail around string with lateral movement; $5=$ escape. The following numbers of animals were analyzed in this task (14). The following number of female mice was used at the age of 6 months: 5XFAD, 11; 5XFAD/hQC, 8; hQC, 11; 5XFAD/QC-KO, 4; QC-KO, 6; wild type, 12.

Statistical Analysis-Statistical differences were evaluated using one-way ANOVA followed by Bonferroni post hoc test or unpaired $t$ test as indicated. All data are given as means \pm S.E. All statistics were calculated using GraphPad Prism version 5.00 software. 


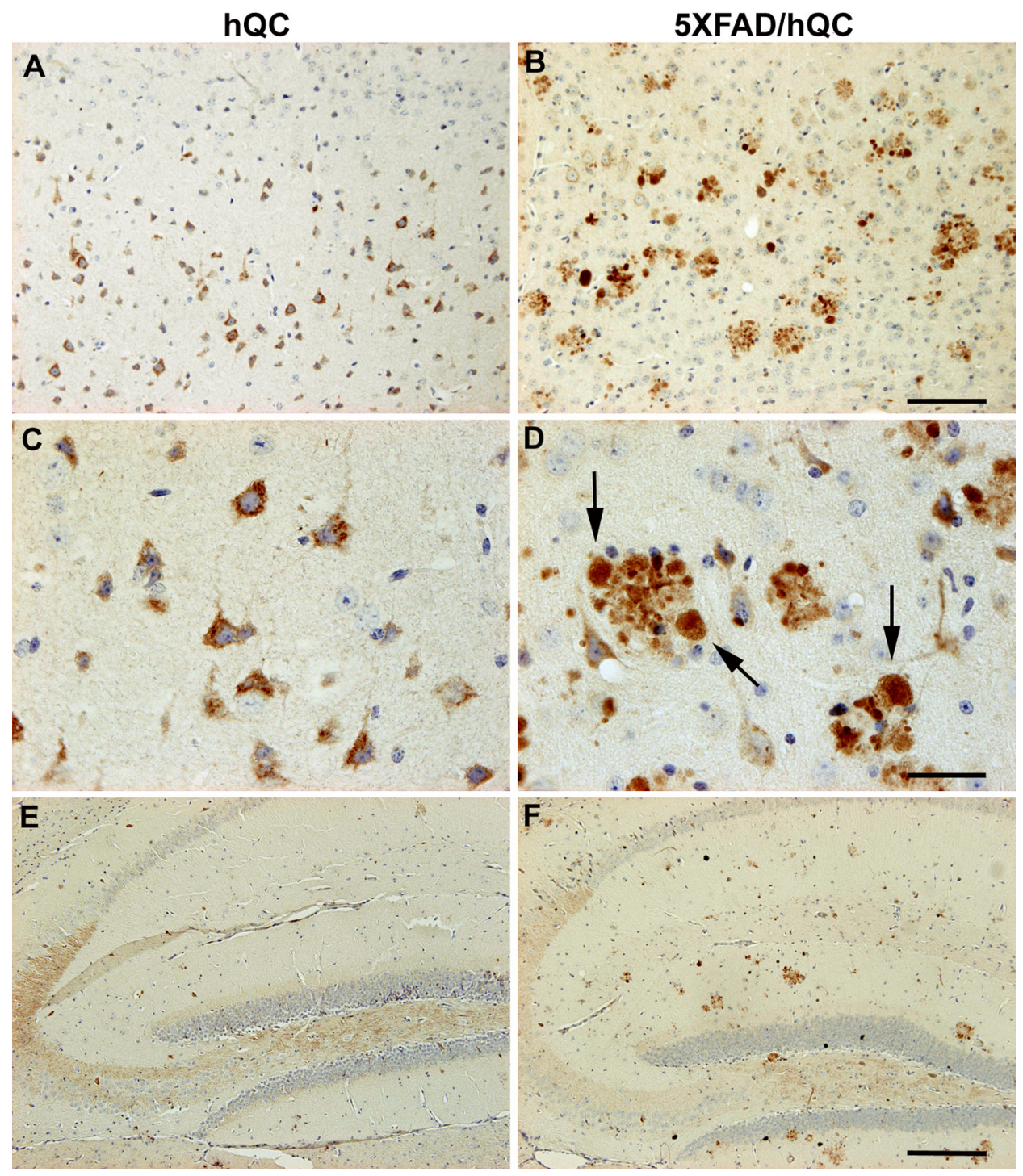

FIGURE 1. Immunohistochemical staining of hQC in hQC and 5XFAD/hQC mice. Expression of human transgenic QC was detected in pyramidal neurons in the cortex of hQC ( $A$ and $C$ ) and 5XFAD/hQC mice and in plaque-associated dystrophic neurites (arrows) of 5XFAD/hQC mice ( $B$ and $D)$. In addition, hQC staining was detected in mossy fibers of the hippocampal formation of hQC and 5XFAD/hQC mice ( $E$ and $F)$. Scale bars, $100 \mu \mathrm{m}(A$ and $B), 50 \mu \mathrm{m}(C$ and $D)$, $200 \mu \mathrm{m}(E$ and $F)$.

\section{RESULTS}

Expression and Distribution of $\mathrm{Q} Q$ in the Brain of $h Q C$ and 5XFAD/hQC Mice-To study human QC overexpression in $\mathrm{hQC}$ transgenic mice (hQC and $5 \mathrm{XFAD} / \mathrm{hQC}$ ), the rabbit polyclonal antiserum 1301 recognizing human QC was used to detect the transgene hQC expression in different brain regions (Fig. 1). Because the expression of the hQC trangene is driven by the Thy1 promoter, abundant pyramidal neurons expressing $\mathrm{hQC}$ were detected in various brain regions of hQC and $5 \mathrm{XFAD} / \mathrm{hQC}$ mice including the cortex (Fig. 1, $A-D$ ), the hippocampus (Fig. 1, $E$ and $F$ ), the midbrain and the cerebellum (data not shown). Notably, a massive staining of hQC was observed in plaque-associated dystrophic neurites in 5XFAD/hQC mice (Fig. $1 D)$. Abundant hQC immunoreactivity was detected in mossy fibers of $\mathrm{hQC}$ and 5XFAD/hQC transgenic mice (Fig. 1, $E$ and $F$ ).
Co-localization of APP and QC in Neurons and the Neuritic Component of Plaques-Double immunofluorescence demonstrated co-localization of hQC and APP in the same cellular compartments in the brain of $5 \mathrm{XFAD} / \mathrm{hQC}$ mice (Fig. 2). APP markedly labels dystrophic neurites around plaques and shows abundant co-localization with hQC suggesting that hQC is axonally transported like APP (Fig. 2).

Effect of hQC Overexpression and QC Knock-out on Plaque Load in the Frontal Cortex of 6-Month-old 5XFAD Mice-The plaque load for total $A \beta$ (NT78) was significantly higher in 5XFAD/hQC compared with 5XFAD mice (5XFAD/hQC, $7.99 \pm 1.1 \%$; and 5XFAD, $4.27 \pm 0.79 \%)$. In 5XFAD/QC-KO mice, the levels were significantly reduced (1.88 $\pm 0.43 \%)$. The plaque load for $\mathrm{A} \beta \mathrm{pE} 3$ showed the same effect. 5XFAD/ hQC (3.08 $\pm 0.44 \%)$ had significantly elevated levels compared with 5XFAD mice $(1.83 \pm 0.31 \%)$ and reduced in $5 \mathrm{XFAD} / \mathrm{QC}-\mathrm{KO}$ mice $(0.55 \pm 0.12 \%)$ (Fig. 3$)$. 

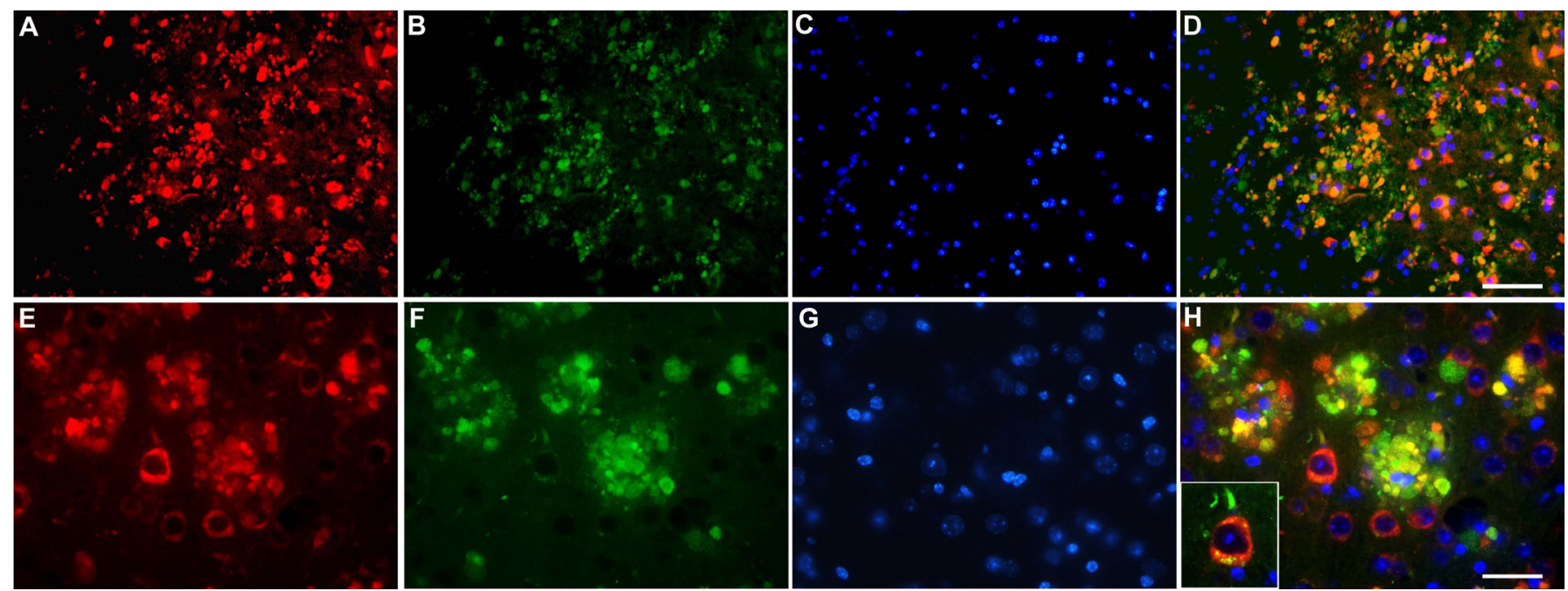

FIGURE 2. Transgene human $Q C$ is co-localized with APP. Double immunostaining in the cortex of $5 X F A D /$ hOC mice using antibodies against APP (red; $A$ and $E$ ), QC (green; $B$ and F) and DAPI (blue; $C$ and G). APP and QC showed co-localization in dystrophic neurites of plaques and in the somatodendritic compartment of pyramidal neurons in the merged images (yellow; $D, H$, inset in $H$ ). Scale bars, $50 \mu \mathrm{m}(A-D), 20 \mu \mathrm{m}(E-H)$.

A

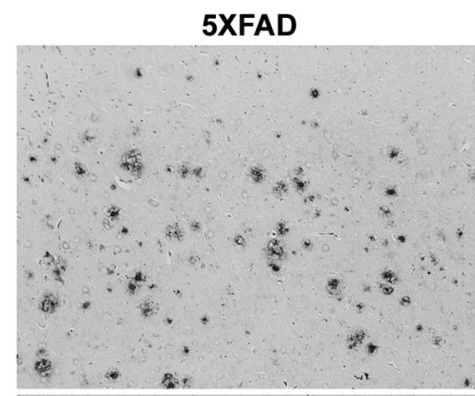

5XFAD/hQC

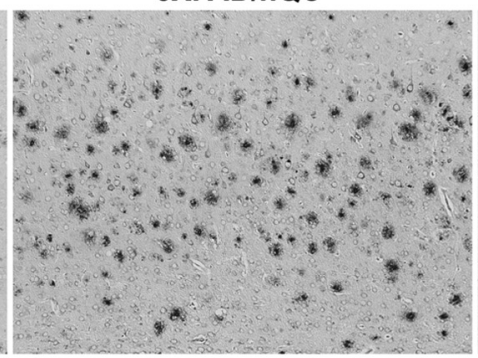

5XFAD/QC-ko

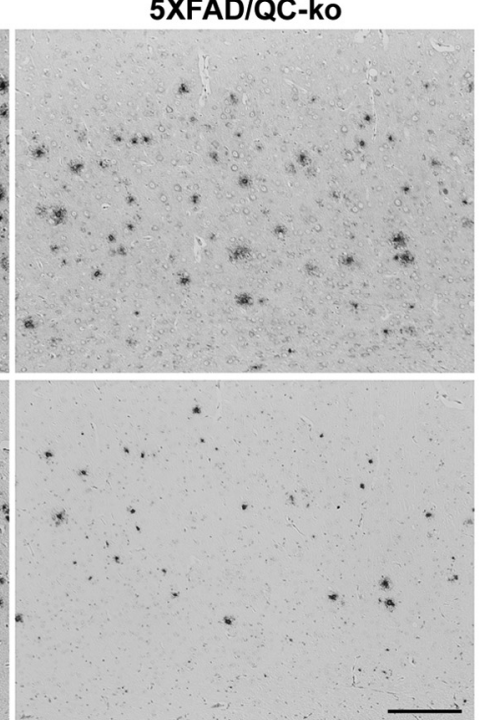

B

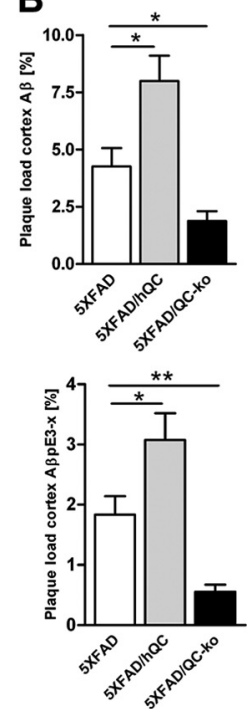

FIGURE 3. Effect of hQC overexpression and QC knock-out on plaque load in 5XFAD mice. $A$, plaque staining in the cortex using antibodies against generic $A \beta$ (NT78) and pyroglutamate-modified $A \beta(2-48)$ in 5 XFAD, 5 XFAD/hQC, and 5XFAD/QC-KO mice. $B$, quantification of plaque load demonstrating significantly elevated $A \beta$ and $A \beta p E 3$ levels in 5 XFAD/hQC and significantly reduced levels in 5 XFAD/QC-KO mouse brain. Scale bar, $200 \mu \mathrm{m} .{ }^{*}, p<0.05 ;{ }^{* *}$, $p<0.01$. Error bars, S.E.

Effect of hQC Overexpression and QC Knock-out on $A \beta x-42$ and AßpE3-42 Levels of 6-Month-old 5XFAD Mice-Protein quantification of $\mathrm{A} \beta \mathrm{x}-42$ (in micrograms/gram of brain weight) and $\mathrm{A} \beta \mathrm{pE} 3-42$ (in nanograms/gram of brain weight) levels in brain lysates of 6-month-old 5XFAD, 5XFAD/hQC, and 5XFAD/QC-KO mice revealed significant differences (Fig. 4). In the SDS+FA-soluble fraction there was a significant $48 \%$ reduction $(p<0.01)$ of $\mathrm{A} \beta \mathrm{x}-42$ levels in $5 \mathrm{XFAD} /$ QC-KO mice (41.07 \pm 4.79$)$ compared with 5XFAD (78.52 \pm 6.54). There was, however, no difference of $A \beta x-42$ levels in the TBS-soluble fraction in $5 X F A D / h Q C ~(0.09 \pm 0.02)$ compared with 5XFAD $(0.12 \pm 0.01)$ and 5 XFAD/QC-KO mice $(0.09 \pm 0.01)$. The effects were more pronounced on A $\beta$ pE3 -42 levels. There was an $86 \%$ elevation $(p<0.01)$ of $\mathrm{A} \beta \mathrm{pE} 3-42$ levels in the TBS-soluble fraction in 5XFAD/hQC $(0.12 \pm 0.02)$ compared with 5XFAD $(0.07 \pm 0.01)$ and unde- tectable levels in 5XFAD/QC-KO mice. In the SDS+FA fraction an $84 \%$ elevation $(p<0.001)$ of $\mathrm{A} \beta \mathrm{pE} 3-42$ levels was found in 5XFAD/hQC (114.9 \pm 5.89$)$ compared with 5XFAD $(62.29 \pm 3.66)$ and significantly reduced levels $(-38 \%)$ in 5XFAD/QC-KO mice (38.59 $\pm 1.93, p<0.01$, compared with 5XFAD) (Fig. 4). Despite the differing A $\beta \mathrm{x}-42$ and $\mathrm{A} \beta \mathrm{pE} 3-42$ levels in 5XFAD, 5XFAD/hQC, and 5XFAD/QC-KO mice, expression levels of transgenic human APP and PS1 were unchanged (supplemental Fig. 2).

Effect of hQC Overexpression and QC Knock-out on Behavioral Performance in 5 XFAD Mice-Motor coordination was assessed by using balance beam and string suspension tasks (Fig. 5, $A$ and $B$ ). In both tasks the $5 \mathrm{XFAD} / \mathrm{hQC}$ performed significantly worse that 5XFAD $(p<0.001$ and $p<0.01$, respectively). Working memory was assessed using $\mathrm{Y}$ - and 54 
A

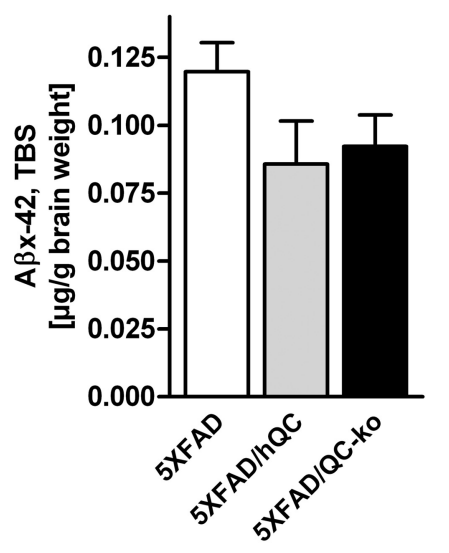

C

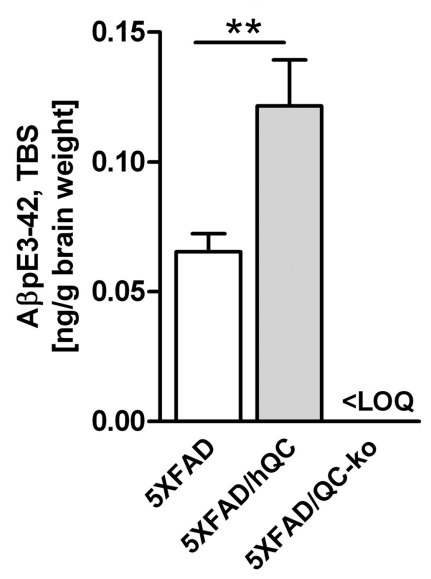

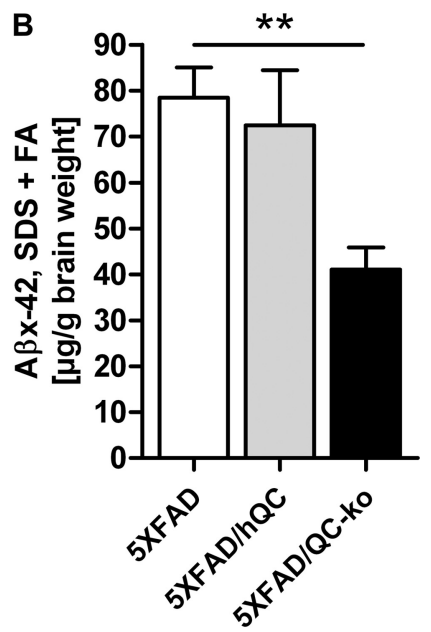

D

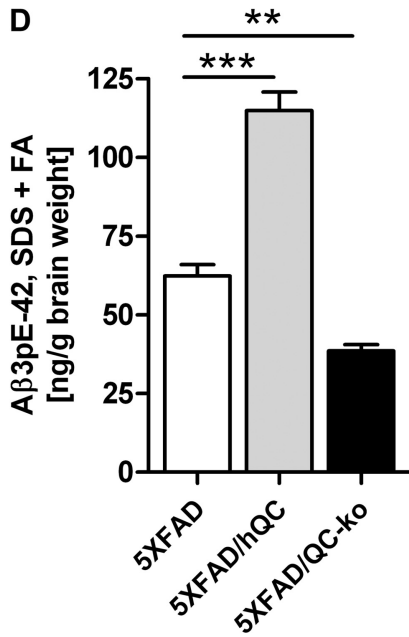

FIGURE 4. Effect of hQC overexpression and $Q C$ knock-out on $A \beta$ levels in 5XFAD mice. Quantification of $A \beta x-42$ and $A \beta p E 3-42$ using ELISA showed significant changes in TBS, SDS, and formic acid (FA) fractions in 5XFAD, 5XFAD/hQC, and 5XFAD/QC-KO mouse brain. SDS and FA fractions were pooled for quantification. $A \beta x-42$ levels were significantly reduced in the SDS+FA fraction of 5 XFAD/QC-KO mice. A $\beta \mathrm{pE} 3-42$ levels were significantly elevated in all fractions in $5 \mathrm{XFAD/hQC}$ mice. Although in the TBS fraction of $5 X F A D / Q C-K O$ mice the levels of $A \beta p E 3-42$ were below the limit of quantitation, in the SDS +FA fractions of 5XFAD/QC-KO mice the levels of $\mathrm{A} \beta \mathrm{pE} 3-42$ were significantly reduced. ${ }^{* *}, p<0.01$; $^{* * *}, p<0.001$. LOQ, limit of quantitation. Error bars, S.E.

a significantly reduced alternation frequency in $5 \mathrm{XFAD} / \mathrm{hQC}$ compared with 5XFAD mice $(p<0.05)$. The number of arm entries during the test period was not different between the groups (Fig. 5, $C$ and $D$ ). Assessment using the more complex cross-maze task demonstrated again a significantly reduced alternation frequency in 5XFAD/hQC compared with 5XFAD mice $(p<0.05)$, and 5XFAD versus wild-type mice $(p<0.05)$. The latter finding corroborated previous results (14). Moreover, the working memory deficit of 5XFAD mice was rescued in 5XFAD/QC-KO mice $(p<0.05)$ showing alternation frequencies indistinguishable from wild-type mice. The number of arm entries during the test period was not different among all groups (Fig. 5, $E$ and $F$ ).

\section{DISCUSSION}

Schilling et al. have shown that cyclization of glutamate at position 3 of $\mathrm{A} \beta$ can be driven enzymatically by QC in vitro

(15). In addition, it has been demonstrated that QC inhibition significantly reduced $\mathrm{A} \beta \mathrm{pE} 3$ formation in vivo, emphasizing the importance of $\mathrm{QC}$ activity during cellular maturation of pyroglutamate-containing peptides. The pharmacological inhibition of QC activity by the QC inhibitor PQ150, which significantly reduced the level of $\mathrm{A} \beta \mathrm{pE} 3$ in vitro (16) and in vivo (10), suggests that $\mathrm{QC}$ inhibition might serve as a new therapeutic approach. Furthermore, the mean level of $A \beta p E 3-$ IgM autoantibodies was significantly decreased in AD patients compared with healthy controls. In the group of mildly cognitive-impaired patients there was a significant positive correlation between $\mathrm{A} \beta \mathrm{pE} 3-\mathrm{IgM}$ and cognitive decline (17).

Interestingly, APP/PS1KI mice, a model with severe neuron loss in the hippocampus, accumulate a large heterogeneity of $\mathrm{N}$-truncated $\mathrm{A} \beta \mathrm{x}-42$ isoforms including $\mathrm{A} \beta \mathrm{pE} 3$ peptides coinciding with the onset of behavioral deficits $(18,19)$. More specifically, transgenic mice expressing only $\mathrm{A} \beta \mathrm{pE} 3-42$ developed a robust and lethal neurological phenotype accompanied by Purkinje cell loss (TBA2 mouse line) (8).

Saido et al. suggested that hypothetically the removal of $\mathrm{N}$-terminal amino acids 1 and 2 of $\mathrm{A} \beta$ might be carried out by amino or dipeptidyl peptidase(s) (6). Aminopeptidase A may be responsible in part for the $\mathrm{N}$-terminal truncation of fulllength $A \beta$ peptides (20).

$\mathrm{N}$-truncated $\mathrm{A} \beta \mathrm{pE} 3$ peptides have been identified by several groups in $\mathrm{AD}$ brains $(5,6,21-32)$. N-terminal deletions in general enhance aggregation of $\beta$-amyloid peptides in vitro (33). A $\beta$ pE3 has a higher aggregation propensity $(34,35)$ and stability (36) and shows an increased toxicity compared with full-length $A \beta$ (37). It has been also suggested that $\mathrm{N}$-truncated $\mathrm{A} \beta$ peptides are formed directly by $\beta$-secretase and not through a progressive proteolysis of full-length $A \beta 1-40 / 42$ (38).

APP transgenic mouse models have been reported to show no (23) or low A $\beta$ pE3 levels (31). Maeda et al. have demonstrated that the localization and abundance of $\left[{ }^{11} \mathrm{C}\right]$ Pittsburgh compound $\mathrm{B}$ autoradiographic signals were closely associated with those of $\mathrm{N}$-terminally truncated and modified $\mathrm{A} \beta \mathrm{pE} 3$ deposition in AD and different APP transgenic mouse brains, implying that the detectability of amyloid by $\left[{ }^{11} \mathrm{C}\right]$ Pittsburgh compound B-positron emission tomography is dependent on the accumulation of specific $A \beta$ subtypes (39). APP/PS1KI $(12,18)$ and 5XFAD $(14)$ mice harbor abundant $A \beta 3 p E$ levels. Interestingly, both models develop an age-dependent neuron loss and robust behavioral deficits, like TBA2 mice with only $\mathrm{A} \beta \mathrm{pE} 3-42$ expression (8).

The findings of the present work are in good agreement with the previous observations that $\mathrm{A} \beta \mathrm{pE} 3-42$ levels correlate with behavioral deficits in transgenic mouse models. Here, we demonstrate for the first time genetic evidence for $\mathrm{QC}$ as a major target for AD. Overexpression of human QC is co-localized with APP in the neuritic component of plaques, leading to elevated $\mathrm{A} \beta \mathrm{pE} 3-42$ levels as detected by ELISA. This finding is corroborated by an increase in the overall plaque pathology including $\mathrm{A} \beta \mathrm{pE} 3-42$ in $5 \mathrm{XFAD} / \mathrm{hQC}$ mice. Consistently, 5XFAD/hQC mice developed a neurological phenotype demonstrated by learning and memory impairments compared with the 5XFAD mouse model at 6 months 

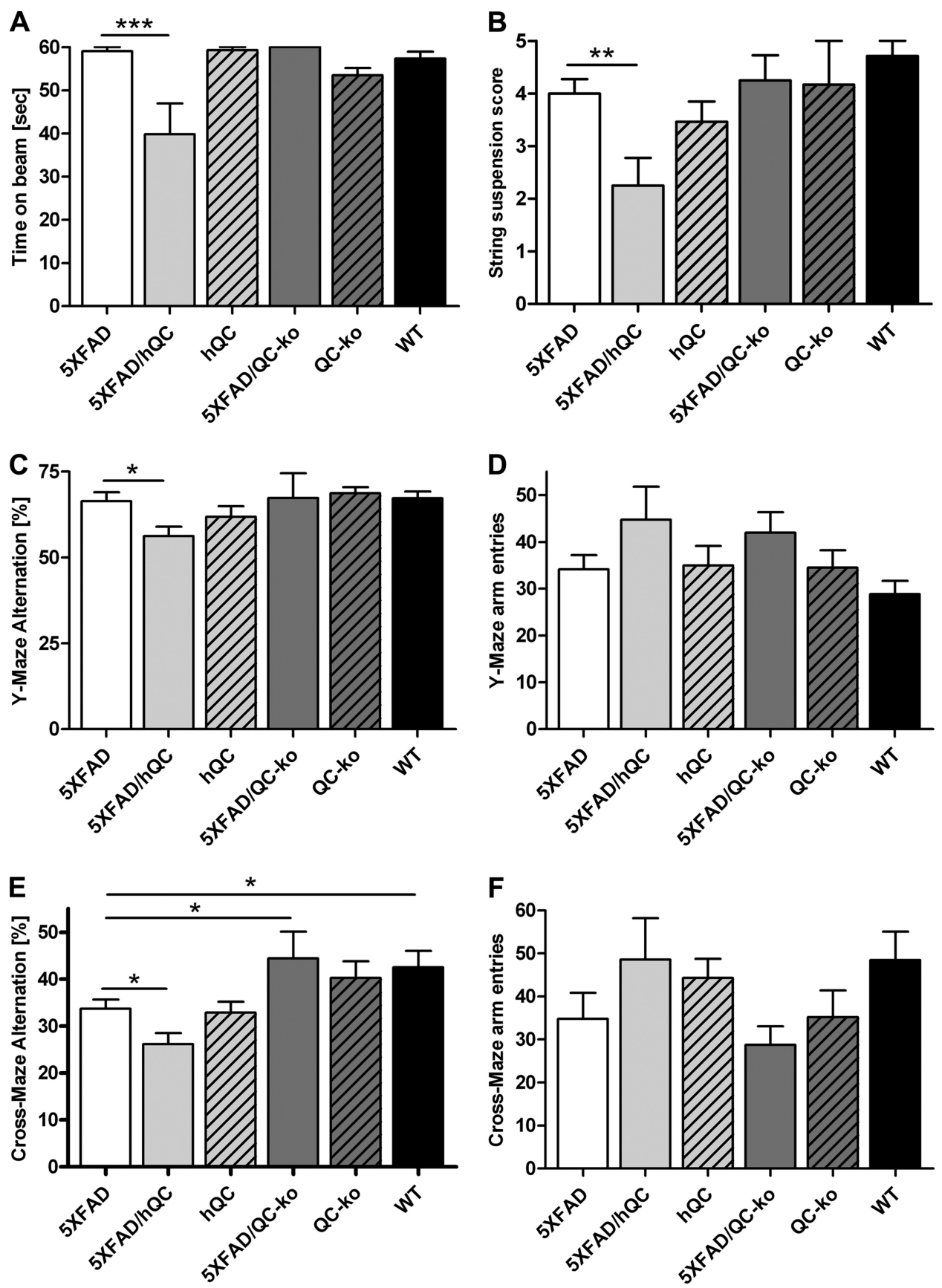

FIGURE 5. Effect of hQC overexpression and QC knock-out on behavioral performance in 5XFAD mice. $A$ and $B, 5 X F A D / h Q C$ mice showed a significantly reduced motor performance in balance beam $(A)$ and string suspension task $(B)$ compared with $5 X F A D$ mice. $C$, in addition, working memory deficits were detected in 5XFAD/hQC compared with 5XFAD mice using Y-and cross-maze (E). Interestingly, the 5XFAD/QC-KO mice showed a rescue of working memory deficits with alternation frequencies indistinguishable from wild-type mice. $D$ and $F$, the number of arm entries in $Y$ - and cross-maze did not differ among the groups. ${ }^{*}, p<0.05 ;{ }^{* *}, p<0.01 ;{ }^{* * *}, p<0.001$.

of age. In addition, we could also show that knock-out of endogenous $\mathrm{QC}$ is sufficient to lower $\mathrm{A} \beta$ levels, including A $\beta \mathrm{pE} 3-42$, leading to a concomitant rescue of behavioral deficits in 5XFAD mice. The apparent discrepancies between $\mathrm{A} \beta \mathrm{x}-42$ and $\mathrm{A} \beta \mathrm{pE} 3-42$ are likely because the $\mathrm{A} \beta \mathrm{x}-42$ peptides are $\sim 1000$ times more abundant than $\mathrm{A} \beta \mathrm{pE} 3-42$ (micrograms versus nanograms/g wet weight). Therefore it is unlikely that an hQC-dependent increase in $\mathrm{A} \beta \mathrm{pE} 3-42$ levels is reflected in a concomitant increase of $\mathrm{A} \beta \mathrm{x}-42$ levels in a stoichiometric manner. It is, however, surprising that the level of $\mathrm{A} \beta \mathrm{x}-42$ is significantly reduced in $5 \mathrm{XFAD} / \mathrm{QC}-\mathrm{KO}$ mice. This might be due to a reduced seeding effect of $\mathrm{A} \beta \mathrm{pE} 3-42$ on full-length $A \beta$, which is therapeutically of interest. It is hypothesized that $\mathrm{A} \beta \mathrm{pE} 3-42$ elevation does not necessarily lead to increased aggregation of $\mathrm{A} \beta \mathrm{x}-42$, which might be due to a saturation effect. In addition, the differences in the plaque load of total $A \beta$ and $A \beta x-42$ levels measured by ELISA might be because plaque load was done in the cortex whereas ELISA was performed in whole brain lysates.

Because $\mathrm{A} \beta \mathrm{pE} 3-42$ levels were not completely reduced, we assume that other $\mathrm{QC}$-related enzymes like iso $\mathrm{QC}$ are responsible for the residual formation of pyroglutamate in QC-KO 
mice. QC and isoQC represent very similar proteins, which are both present in the secretory pathway of cells. The functions of QCs and isoQC complement each other, suggesting a pivotal role of pyroglutamate modification for protein and peptide maturation (40). To analyze a possible contribution of isoQC to the remaining QC-like activity in 5XFAD/QC-KO mice, we performed a Western blot analysis using an isoQC antibody. The protein levels of isoQC were unchanged in different brain regions between WT and QC-KO mice. This observation demonstrates that the finding of residual pyroglutamate $\mathrm{A} \beta$ levels in 5XFAD/QC-KO mice is likely mediated by isoQC (supplemental Fig. 3). In conclusion, reduction of QC was sufficient to rescue the behavioral impairments in the 5XFAD mouse model suggesting a crucial role of QC as a therapeutic target for AD.

Acknowledgments-We thank Petra Tucholla, Katrin Schulz and Eike Scheel for technical support.

\section{REFERENCES}

1. Selkoe, D. J. (1998) Trends Cell Biol. 8, 447-453

2. Masters, C. L., Simms, G., Weinman, N. A., Multhaup, G., McDonald, B. L., and Beyreuther, K. (1985) Proc. Natl. Acad. Sci. U.S.A. 82, 4245-4249

3. Selkoe, D. J., Abraham, C. R., Podlisny, M. B., and Duffy, L. K. (1986) J. Neurochem. 46, 1820-1834

4. Gorevic, P. D., Goñi, F., Pons-Estel, B., Alvarez, F., Peress, N. S., and Frangione, B. (1986) J. Neuropathol. Exp. Neurol. 45, 647-664.

5. Mori, H., Takio, K., Ogawara, M., and Selkoe, D. J. (1992) J. Biol. Chem. 267, 17082-17086

6. Saido, T. C., Iwatsubo, T., Mann, D. M., Shimada, H., Ihara, Y., and Kawashima, S. (1995) Neuron 14, 457-466

7. Portelius, E., Bogdanovic, N., Gustavsson, M. K., Volkmann, I., Brinkmalm, G., Zetterberg, H., Winblad, B., and Blennow, K. (2010) Acta Neuropathol. 120, 185-193

8. Wirths, O., Breyhan, H., Cynis, H., Schilling, S., Demuth, H. U., and Bayer, T. A. (2009) Acta Neuropathol. 118, 487-496

9. Cynis, H., Scheel, E., Saido, T. C., Schilling, S., and Demuth, H. U. (2008) Biochemistry 47, 7405-7413

10. Schilling, S., Zeitschel, U., Hoffmann, T., Heiser, U., Francke, M., Kehlen, A., Holzer, M., Hutter-Paier, B., Prokesch, M., Windisch, M., Jagla, W., Schlenzig, D., Lindner, C., Rudolph, T., Reuter, G., Cynis, H., Montag, D., Demuth, H. U., and Rossner, S. (2008) Nat. Med. 14, $1106-1111$

11. Oakley, H., Cole, S. L., Logan, S., Maus, E., Shao, P., Craft, J., GuillozetBongaarts, A., Ohno, M., Disterhoft, J., Van Eldik, L., Berry, R., and Vassar, R. (2006) J. Neurosci. 26, 10129-10140

12. Wirths, O., Bethge, T., Marcello, A., Harmeier, A., Jawhar, S., Lucassen, P. J., Multhaup, G., Brody, D. L., Esparza, T., Ingelsson, M., Kalimo, H., Lannfelt, L., and Bayer, T. A. (2010) J. Neural Transm. 117, 85-96

13. Hartlage-Rübsamen, M., Staffa, K., Waniek, A., Wermann, M., Hoffmann, T., Cynis, H., Schilling, S., Demuth, H. U., and Rossner, S. (2009) Int. J. Dev. Neurosci. 27, 825-835

14. Jawhar, S., Trawicka, A., Jenneckens, C., Bayer, T. A., and Wirths, O. (2010) Neurobiol. Aging, doi:10.1016/j.neurobiolaging.2010.05.027

15. Schilling, S., Hoffmann, T., Manhart, S., Hoffmann, M., and Demuth, H. U. (2004) FEBS Lett. 563, 191-196

16. Cynis, H., Schilling, S., Bodnár, M., Hoffmann, T., Heiser, U., Saido,
T. C., and Demuth, H. U. (2006) Biochim. Biophys. Acta 1764, $1618-1625$

17. Marcello, A., Wirths, O., Schneider-Axmann, T., Degerman-Gunnarsson, M., Lannfelt, L., and Bayer, T. A. (2009) Neurobiol. Aging, doi:10.1016/j.neurobiolaging.2009.08.011

18. Casas, C., Sergeant, N., Itier, J. M., Blanchard, V., Wirths, O., van der Kolk, N., Vingtdeux, V., van de Steeg, E., Ret, G., Canton, T., Drobecq, H., Clark, A., Bonici, B., Delacourte, A., Benavides, J., Schmitz, C., Tremp, G., Bayer, T. A., Benoit, P., and Pradier, L. (2004) Am. J. Pathol. $165,1289-1300$

19. Breyhan, H., Wirths, O., Duan, K., Marcello, A., Rettig, J., and Bayer, T. A. (2009) Acta Neuropathol. 117, 677-685

20. Sevalle, J., Amoyel, A., Robert, P., Fournié-Zaluski, M. C., Roques, B., and Checler, F. (2009) J. Neurochem. 109, 248-256

21. Saido, T. C., Yamao-Harigaya, W., Iwatsubo, T., and Kawashima, S. (1996) Neurosci. Lett. 215, 173-176

22. Kuo, Y. M., Emmerling, M. R., Woods, A. S., Cotter, R. J., and Roher, A. E. (1997) Biochem. Biophys. Res. Commun. 237, 188-191

23. Kuo, Y. M., Kokjohn, T. A., Beach, T. G., Sue, L. I., Brune, D., Lopez, J. C., Kalback, W. M., Abramowski, D., Sturchler-Pierrat, C., Staufenbiel, M., and Roher, A. E. (2001) J. Biol. Chem. 276, 12991-12998

24. Hosoda, R., Saido, T. C., Otvos, L., Jr., Arai, T., Mann, D. M., Lee, V. M., Trojanowski, J. Q., and Iwatsubo, T. (1998) J. Neuropathol. Exp. Neurol. 57, 1089-1095

25. Harigaya, Y., Saido, T. C., Eckman, C. B., Prada, C. M., Shoji, M., and Younkin, S. G. (2000) Biochem. Biophys. Res. Commun. 276, 422-427

26. Iwatsubo, T., Saido, T. C., Mann, D. M., Lee, V. M., and Trojanowski, J. Q. (1996) Am. J. Pathol. 149, 1823-1830

27. Miravalle, L., Calero, M., Takao, M., Roher, A. E., Ghetti, B., and Vidal, R. (2005) Biochemistry 44, 10810-10821

28. Piccini, A., Russo, C., Gliozzi, A., Relini, A., Vitali, A., Borghi, R., Giliberto, L., Armirotti, A., D’Arrigo, C., Bachi, A., Cattaneo, A., Canale, C., Torrassa, S., Saido, T. C., Markesbery, W., Gambetti, P., and Tabaton, M. (2005) J. Biol. Chem. 280, 34186-34192

29. Piccini, A., Zanusso, G., Borghi, R., Noviello, C., Monaco, S., Russo, R., Damonte, G., Armirotti, A., Gelati, M., Giordano, R., Zambenedetti, P., Russo, C., Ghetti, B., and Tabaton, M. (2007) Arch. Neurol. 64, 738-745

30. Russo, C., Saido, T. C., DeBusk, L. M., Tabaton, M., Gambetti, P., and Teller, J. K. (1997) FEBS Lett. 409, 411-416

31. Güntert, A., Döbeli, H., and Bohrmann, B. (2006) Neuroscience 143, 461-475

32. Tekirian, T. L., Saido, T. C., Markesbery, W. R., Russell, M. J., Wekstein, D. R., Patel, E., and Geddes, J. W. (1998) J. Neuropathol. Exp. Neurol. 57, $76-94$

33. Pike, C. J., Overman, M. J., and Cotman, C. W. (1995) J. Biol. Chem. 270, 23895-23898

34. He, W., and Barrow, C. J. (1999) Biochemistry 38, 10871-10877

35. Schilling, S., Lauber, T., Schaupp, M., Manhart, S., Scheel, E., Böhm, G., and Demuth, H. U. (2006) Biochemistry 45, 12393-12399

36. Kuo, Y. M., Webster, S., Emmerling, M. R., De Lima, N., and Roher, A. E. (1998) Biochim. Biophys. Acta 1406, 291-298

37. Russo, C., Violani, E., Salis, S., Venezia, V., Dolcini, V., Damonte, G., Benatti, U., D'Arrigo, C., Patrone, E., Carlo, P., and Schettini, G. (2002) J. Neurochem. 82, 1480-1489

38. Russo, C., Salis, S., Dolcini, V., Venezia, V., Song, X. H., Teller, J. K., and Schettini, G. (2001) Neurobiol. Dis. 8, 173-180

39. Maeda, J., Ji, B., Irie, T., Tomiyama, T., Maruyama, M., Okauchi, T., Staufenbiel, M., Iwata, N., Ono, M., Saido, T. C., Suzuki, K., Mori, H., Higuchi, M., and Suhara, T. (2007) J. Neurosci. 27, 10957-10968

40. Stephan, A., Wermann, M., von Bohlen, A., Koch, B., Cynis, H., Demuth, H. U., and Schilling, S. (2009) FEBS J. 276, 6522-6536 


\section{Supplementary Information Jawhar et al.}

\section{Real time-PCR}

For real-time RT-PCR analysis six-month-old female mice were used (5XFAD, 5XFAD/hQC, 5XFAD/QC-ko). Mice were killed by $\mathrm{CO}_{2}$ asphyxiation, brain hemispheres were carefully dissected, snap frozen in liquid nitrogen and stored at $-80{ }^{\circ} \mathrm{C}$ until further analysis. Deep frozen brain hemispheres were homogenized in $1 \mathrm{ml}$ of Trizol reagent (Invitrogen) per $100 \mathrm{mg}$ tissue using a glass-teflon homogenizer (10 strokes, $800 \mathrm{rpm})$. RNA extraction was performed according to the protocol of the manufacturer. DNAse treatment and Reverse transcription of RNA of the purified RNA samples was carried out using QuantiTect Reverse Transcription Kit (Qiagen, Hilden, Germany) according to the protocol of the supplier. RTPCR was performed using a Stratagene MX3000P Real-Time Cycler. The SYBR-green based DyNAmo Flash SYBR Green qPCR Kit (Finnzymes) containing ROX as an internal reference dye was used for amplification. Primer sets detecting murine QC (mQC) and $\beta$-Actin were purchased from Qiagen (QuantiTect Primer Assays: murine QC: Mm_Qpct_3_SG (Amplicon length: 117 bp); murine $\beta$-Actin: Actb_1_SG (Amplicon length: 149 bp, Qiagen, Hilden, Germany). The resulting PCR products from the RT-PCR were in addition run on a $2.5 \%$ agarose gel.

\section{Western Blot}

$20 \mu \mathrm{g}$ of the brain lysates per lane was loaded on $4-12 \%$ sodium dodecylsulfate polyacrylamide gels (Vario-Gel, Anamed, Germany) and run at $150 \mathrm{~V}$ for 1 hour. Proteins were transferred to a nitrocellulose membrane for 2 hours at $25 \mathrm{~V}$ using wet transfer. After transfer, membrane was blocked in $10 \%$ non-fat dry milk in TBS containing $0.05 \%$ Tween (TBS-T) for 1 hour. The membrane was then incubated overnight using mouse monoclonal antibody W0-2 (1:5000, Millipore) to detect APP, polyconal antiserum 3109 against PS1 (1:1000, generous gift from Prof. Jochen Walter, Bonn), polyclonal antiserum 5407 against isoQC (1:1000, Probiodrug) and mouse monoclonal antibody detecting $\beta$-Actin ( $\beta$-Actin, 1:5000, Sigma). The bound antibodies were detected by secondary HRP-conjugated antibodies (DAKO) followed by enhanced chemiluminescence. 
Supplementary Figure 1: Absence of $\mathrm{mQC}$ in the 5XFAD/QC-ko mice. (A) RT PCR was used to compare the expression level of murine QC in 5XFAD, 5XFAD/hQC and 5XFAD/QC-ko. No mQC expression could be detected in 5XFAD/QC-ko mice. The very late rise in fluorescence is due to the formation of a primer dimer in the absence of the target sequence. This has been verified by melting curve analysis (not shown), as well as by agarose gel electrophoresis (B). RT PCR for the $\beta$-Actin was used as a control for the amount and integrity of the cDNA in the samples.

A

5XFAD

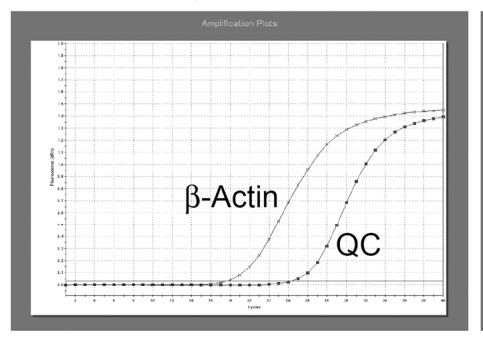

5XFAD/hQC

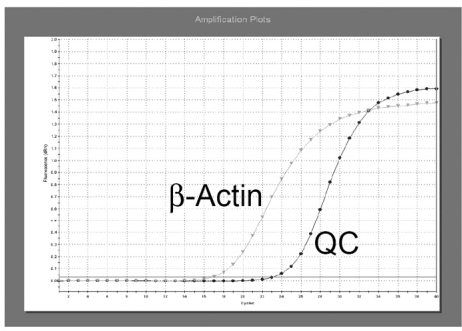

5XFAD/QC knock-out

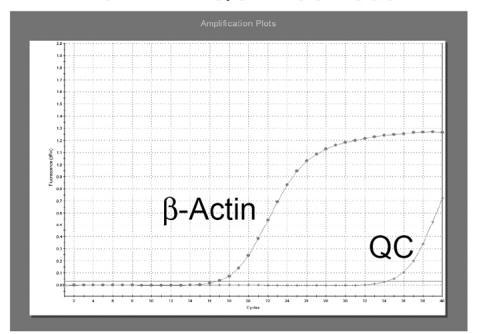

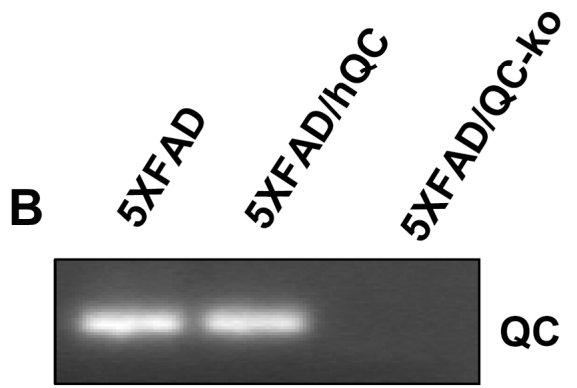

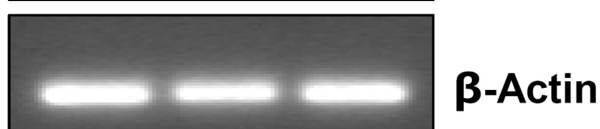


Supplementary Figure 2: QC overexpression and QC knockout did not affect amyloid precursor protein (APP) or presenilin 1 (PS1) levels in 5XFAD mice. Western blot using W0-2 detecting APP and antiserum 3109 detecting PS1 revealed no difference in the expression levels of both proteins among 5XFAD, 5XFAD/hQC and 5XFAD/QC-ko mice. $\beta$ actin was used as a loading control.

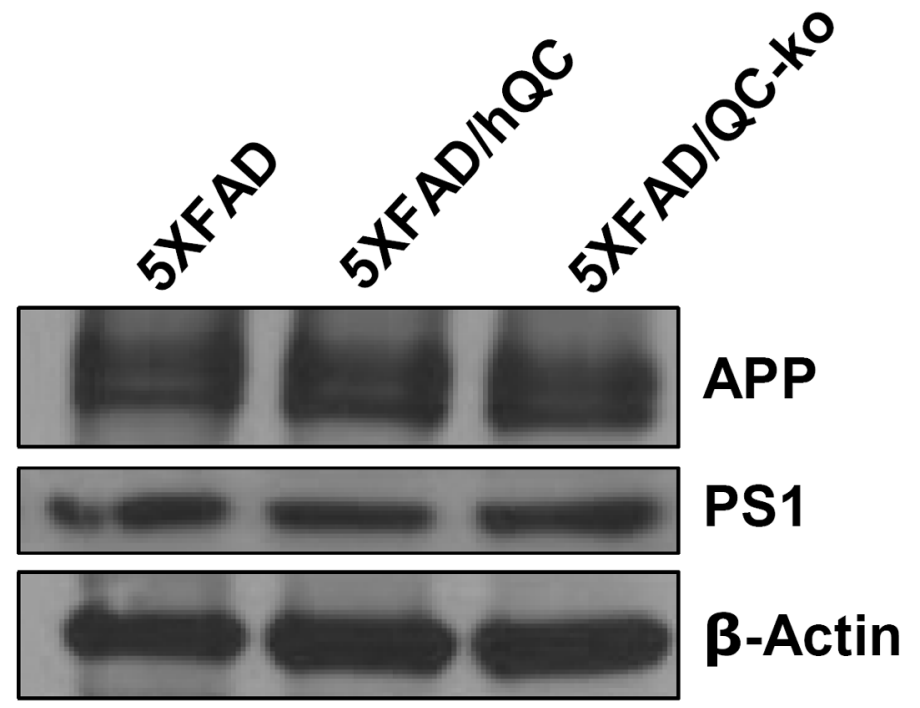

Supplementary Figure 3: Unchanged expression levels of isoQC in WT and QC-ko mice. Western blot analysis of isoQC in different brain regions in WT and QC-ko mice. Recombinant murine isoQC ( $\mathrm{m}$-isoQC) was used as a positive control.

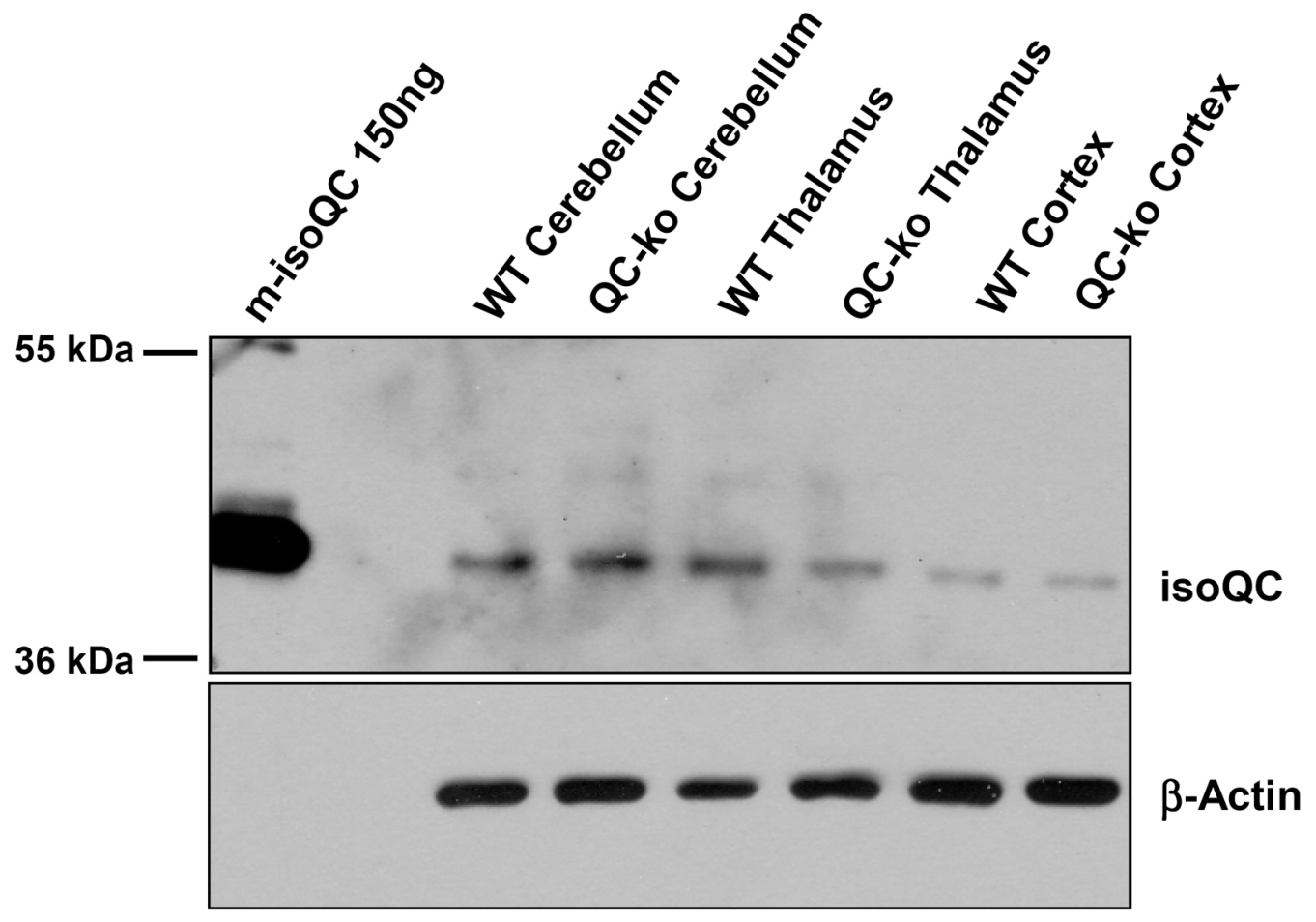




\section{Discussion}

\subsection{Project I: "Characterization of the 5XFAD mouse model"}

Over the past years, many mouse models have been generated based on FAD mutations to mimic AD pathology. These models contributed widely to our understanding of the changes occurring on molecular, tissue and clinical levels. Moreover, they represent essential tools for preclinical testing of therapeutic approaches. However, most of these models are limited either by harboring mild or late pathology or lacking some of the cardinal AD hallmarks. The introduction of multiple FAD mutations in APP and PS1 in transgenic mice aimed at accelerating and increasing the $\mathrm{AD}$ pathology to resemble that found in $\mathrm{AD}$ patients.

The 5XFAD mouse model is a double transgenic APP/PS1 mouse model that expresses five FAD mutations leading to a higher $\mathrm{A} \beta_{\mathrm{x}-42}$ level and accelerated amyloid deposition (Oakley et al., 2006). The co-expression of five FAD mutations results in early multiple AD pathological hallmarks. As this model lacks NFTs formation, it represents an amyloid-predominant model of AD. The 5XFAD mouse model shares this accelerated aggressive AD pathology with other APP/PS1 AD models such as APP/PS1KI (Casas et al., 2004) and APP/PS1 (Schmitz et al., 2004). However, the 5XFAD mice are particularly interesting due to the co-integration of the APP and PS1 transgenes at the same genetic locus and thus the co-segregation of the transgenes. This facilitates breeding and genotyping and more importantly enables easy generation of bigenic mouse models.

The 5XFAD mice were originally generated and described on a B6/SJL-hybrid background. They were reported to develop early plaque deposition associated with gliosis in addition to neuron and synaptic loss and memory deficits (Oakley et al., 2006). The objectives of the present study were to validate and extend the findings in the 5XFAD mouse model after being backcrossed with C57BL/6J mice, to facilitate the comparison with other models which are mostly kept on the C57BL/6J genetic background. This is particularly important because the genetic background has been reported to have potential influence on the APP processing, the behavioral performance, the neuropathology and the response to excitotoxicity (Carlson et al., 1997; Lehman et al., 2003; Lassalle et al., 2008; Glazner et al., 2010). 


\subsubsection{The 5XFAD mouse model shows age-dependent neurological deficits and motor impairments}

$\mathrm{AD}$ is characterized by progressive cognitive impairments in addition to neuropsychiatric symptoms such as anomalous motor behavior, depression, weight loss, irritability and agitation. In the current study, the neurological deficits and the motor performance in the 5XFAD mice were investigated.

The 5XFAD mice exhibited reduced body weight in comparison to their wildtype littermates starting from 9 months of age. Weight loss occurs frequently in AD patients (Tamura et al., 2007) and in some AD mouse models such as the TASTPM (Pugh et al., 2007). Moreover, 12-month-old 5XFAD mice showed an abnormal extension reflex by retracting hind- and fore-paws simultaneously when suspended by the tail. This abnormal reflex has been correlated with pathology in the spinal cord, cerebellum, basal ganglia, forebrain, hindbrain and midbrain in other models. It has been described in several AD models such as APP23 (Lalonde et al., 2005), Tg2576 (Lalonde et al., 2003b; Seo et al., 2010) and APP/PS1KI mouse models (Wirths et al., 2007).

In line with the clasping behavior, the motor phenotype started to become prominent in 5XFAD mice at 9 months of age. This was indicated using the balance beam, which reflects balance and general motor abilities (Arendash et al., 2001a) and the string suspension task, which reflects agility and grip capacity (Moran et al., 1995). Impairments in motor coordination might be due to pathology in different CNS regions such as the cerebellum, the basal ganglia, the neocortex and the spinal cord (Lalonde and Strazielle, 2007; Wirths and Bayer, 2008). However, due to the use of the Thy- 1 promoter construct, the transgene expression in the cerebellum is restricted. Therefore, axonal deficits, loss of connecting neurons with the cerebellum and/or impairment of other brain regions, like the motor cortex and the basal ganglia, might account for the described motor phenotype. Motor deficits in these tasks or other motor tasks have been detected in some AD mouse model such as APP single transgenic mice like Tg2576 (Arendash et al., 2001b), APP23 (Van Dam et al., 2003) and Tg-APP mice (Sw, V717F) (Lee et al., 2004) and double transgenic APP/PS1 mice such as APP+PS1 (Ewers et al., 2006). The assessment of motor impairment is a highly relevant 
issue, as some reference memory tasks like the Morris water maze are severely influenced by any disturbance in motor performance, leading to erroneous results.

Motor impairments are widely described in $\mathrm{AD}$ patients and are already present at early stages of the disease in $13 \%$ of the patients at the first examination, progressing to $36 \%$ at the last examination. These signs included poverty in speech/facial expression, rigidity, posture/gait disturbances and bradykinesia (Scarmeas et al., 2004). Gait disturbance was reported in $40 \%$ of $\mathrm{AD}$ patients in comparison to $18 \%$ of age-matched control patients (O'Keeffe et al., 1996). Prospective studies suggested the motor impairments to have a predictive value for the cognitive decline in $\mathrm{AD}$ and to be useful in identifying MCI patients at risk of developing AD (Chui et al., 1994; Aggarwal et al., 2006).

Taken together, the 5XFAD mice show age-dependent neurological and motor deficits which mimic common phenotypes seen in $\mathrm{AD}$ patients and found in some $\mathrm{AD}$ mouse models.

\subsubsection{The 5XFAD mouse model shows early pathological deficits in the spinal cord}

Impairments in axonal transport have been described in AD patients (Stokin et al., 2005) and many AD and DS mouse models to variable extents (Stokin et al., 2005; Salehi et al., 2006; Wirths et al., 2006; Wirths et al., 2007; Coma et al., 2010). As mentioned above, motor impairments are quite common at different stages of $\mathrm{AD}$, raising the question whether axonal transport deficits might contribute to these impairments.

To elucidate whether the clasping phenotype and the motor impairments of the 5XFAD mice coincide with axonal deficits, screening for axonal swellings in the spinal cord using a marker for neurofilaments (NF200), which are the most abundant fibrillar component of the axons, was performed. Plaque-independent axonal spheroids were quantified in an agedependent manner because they may represent disturbance in the axonal transport rather than local toxic effects of plaques. However, staining against neurofilaments together with $A \beta$ peptides revealed many axonal swellings in the form of small dystrophic neurites decorating the neuritic plaques.

Axonal spheroids were present mainly in the gray matter as early as 3 months of age and showed age-dependent increase, a pattern that was also seen in the pons. In line with our findings, dystrophic neurites and plaque-independent axonal spheroids have been recently 
detected in the brain of 5XFAD mice as early as 2 months of age (Zhang et al., 2009). Axonal swellings have been previously reported in other $\mathrm{AD}$ transgenic mouse models such as APP/PS1KI and APP/PS1 to a variable extent (Wirths et al., 2006; Wirths et al., 2007). They were also reported in tau-based (Spittaels et al., 1999; Probst et al., 2000) or APOE-based models (Tesseur et al., 2000) which also show different forms of motor deficits. In good agreement, APP-positive spheroids were demonstrated in the spinal cord of early-onset AD patients, a finding that may contribute to the understanding of the motor deficits seen in $\mathrm{AD}$ (Wirths et al., 2007).

Previous studies in APP/PS1 mouse models suggested that intraneuronal $\mathrm{A} \beta$ contributes to the axonal impairments in these mice (Wirths et al., 2006; Wirths et al., 2007; Chen et al., 2011). Interestingly, intraneuronal $A \beta$ was detected within the spinal cord motor neurons of the 5XFAD mice as early as 3 months of age, which might be involved in the axonal transport impairment. This is supported by the finding that $A \beta_{x-42}$ accumulates in lumbar motor neurons of patients suffering from amyotrophic lateral sclerosis (ALS) (Calingasan et al., 2005), a disease in which axonal transport deficits are clearly implicated (Chevalier-Larsen and Holzbaur, 2006).

Although many studies investigated the tau pathology in the spinal cords of AD patients (Saito and Murayama, 2000), few studies analyzed the presence of amyloid pathology in this region (Bugiani et al., 1989; Ogomori et al., 1989). Plaque deposits in the spinal cord have been recently described in AD mouse models such as Tg2576 (Seo et al., 2010). To evaluate the plaque pathology in the spinal cord of the 5XFAD mouse model, plaque load was quantified in the gray matter of the spinal cord of 3-, 6- and 12-month-old mice and showed a significant increase with age. This pattern has also been reported in other APP/PS1 models (Wirths et al., 2006; Wirths et al., 2007). In both models, the presence of plaque pathology in the spinal cord was preceded by the presence of intraneuronal A $\beta$. Although earlier time points were not investigated in the current project, work from Zhang et al. (2009) indicated that intracellular $A \beta$ and axonal swellings existed as early as 1 month of age and preceded the plaque deposition in the brain of 5XFAD mice. Moreover, recent data from an APP/PS1 model supports the current findings. It was shown that intraneuronal $A \beta$ preceded plaque 
deposition which correlated well with the axonal alterations in the corpus callosum and the anterior commissure of APP/PS1 mice (Chen et al., 2011).

In summary, the current work sheds light on the neurological symptoms, motor impairments, axonopathy and spinal cord pathology in the 5XFAD mouse model which have not been assessed before. Accumulation of intraneuronal $A \beta$ coincides with the axonal impairments in the spinal cord of 5XFAD mice that are represented by axonal spheroids and motor phenotype. Nevertheless, this does not rule out that pathological changes in other regions involved in motor activity may also contribute to the neurological and motor phenotype seen in the 5XFAD mice.

\subsubsection{Neuron loss in 5XFAD mice correlates with the presence of intraneuronal A $\beta$}

Neuron loss and atrophy are among the major neuropathological hallmarks seen in AD patients, and their extent and distribution depends on the severity of the disease. Among the various $\mathrm{AD}$ mouse models, only a few exhibit neuron loss, and they are mostly those which harbor multiple FAD mutations (Wirths and Bayer, 2010). Loss of neurons was previously assumed in 9-month-old 5XFAD mice, however, this was not appropriately quantified (Oakley et al., 2006; Ohno et al., 2007). For this reason, designed-based stereology was utilized to quantify neurons in the CA1 region and the overall frontal cortex of 12-month-old 5XFAD mice. No significant neuron loss could be detected in these regions. The absence of neuron loss in CA1 was supported by a very recent paper by Shao and colleagues who reported no neuron loss in the CA1 region of 6-month-old 5XFAD mice (Shao et al., 2011).

The fifth cortical layer was specifically studied, which, together with the subiculum, was previously assumed to have neuron loss (Oakley et al., 2006). In agreement, 12-monthold 5XFAD females showed about $40 \%$ less neuron in the fifth cortical layer of the frontal cortex compared to wildtype littermates. In line with our findings in the fifth cortical layer, a very recent paper by Lemmens and colleagues revealed similar findings in APP/PS1KI mice. The authors found a 34\% neuron loss in V-VI layers of the frontal cortex of 10-month-old APP/PS1KI mice compared to 2-month-old mice, while no neuron loss could be detected in the I-IV layers of the frontal cortex (Lemmens et al., 2011). 
The frontal cortex is among the brain regions that are known to be affected in $\mathrm{AD}$ (Braak et al., 1996) contributing to the memory, behavioral and cognitive symptoms seen in AD patients. Pyramidal neurons in the fifth cortical layers are particularly important for corticospinal, corticostriatal and corticocortical connections (Bussiere et al., 2003; Anderson et al., 2010). These neurons have been reported to be particularly vulnerable in the course of AD (Morrison and Hof, 2007).

Growing evidence compiled during the last years points to the role played by intraneuronal $\mathrm{A} \beta$ in the neurodegeneration process in $\mathrm{AD}$ (Gouras et al., 2010; Bayer and Wirths, 2011). In this regard, accumulation of intraneuronal $A \beta$ in the hippocampus and the cortex was investigated. The major difference between the fifth cortical layer and the CA1 was the intraneuronal accumulation of $A \beta$, although both regions expressed comparable levels of the APP immunoreactivity and presented extracellular amyloid deposition. Intraneuronal $\mathrm{A} \beta$ accumulation was prominent in the pyramidal neurons of the fifth cortical layer in comparison to other cortical layers, which is due to the Thy-1 driven transgene expression pattern (Feng et al., 2000). This might account for the discrepancy in the neuron loss between the overall frontal cortex and the fifth cortical layer. The observations in the 5XFAD mice confirm previous findings in APP/PS1KI mice, where neuron loss occurred only in brain regions showing intraneuronal $A \beta$ accumulation, like the frontal cortex, CA1 or distinct cholinergic brain stem nuclei. In the thalamus or the striatum, regions where only extracellular plaques were detectable, no neuron loss could be demonstrated (Casas et al., 2004; Schmitz et al., 2004; Christensen et al., 2008; Breyhan et al., 2009) arguing against a significant role of extracellular plaques in terms of $\mathrm{A} \beta$ toxicity. In addition, data from a new APP transgenic mouse model harboring the E693 $\Delta$ mutation support these observations. This model does not show any extracellular amyloid deposits even at 24 months of age. However, it demonstrates CA3 neuron loss at this time point which is preceded by intraneuronal $A \beta$ oligomer accumulation (Tomiyama et al., 2010). Recently, the use of a novel gene transfer technique with a lentiviral $A \beta_{1-42}$ resulted in intracellular but not extracellular $A \beta$ accumulations in the targeted rat primary motor cortex. Expression of intracellular $A \beta_{1-42}$ led to pathological changes seen in human AD brains, including cell death (Rebeck et al., 2010). On the contrary, some studies suggest a correlation between neuron loss and extracellular plaque deposition. 
Triple immunostaining combined with confocal microscopy showed dramatic focal neuronal toxicity associated primarily with fibrillar A $\beta$ deposits in AD and PSAPP mice. These results led the authors to suggest that $\mathrm{A} \beta$ develops neurotoxic properties in vivo when it adopts a fibrillar $\beta$-pleated sheet conformation (Urbanc et al., 2002).

Interestingly, intraneuronal $A \beta$ in the fifth cortical layer showed strong immunoreactivity at 3 months of age which decreased with aging until it was almost absent at 12 months of age. This observation can be due to the neuron death occurring in cells harboring $A \beta$ leading to a loss of intraneuronal $A \beta$ signal with age. A similar pattern has been reported in the TBA2.1 mouse model were death of the neurons was associated with reduced intraneuronal $A \beta$ signal (Alexandru et al., 2011).

Altogether, findings from the current work confirm and quantify neuron loss described previously in the 5XFAD mice on a different genetic background. Also, it strongly suggests that neuron loss draws a parallel to the intraneuronal $A \beta$ observed in specific brain regions in this mouse model.

\subsubsection{Spatial working memory impairment in the 5XFAD mouse model}

$\mathrm{AD}$ is an amnesic disorder with multiple forms of memory being affected, including the working memory. In rodents, spatial working memory evaluates recent memories related to their foraging behavior in the wild (Dember and Fowler, 1958). It has been proven that the hippocampus, the prefrontal cortex and the hippocampal-prefrontal cortical circuit are important for working memory (Divac, 1975); and these brain areas are among the regions known to be affected in AD. Oakley et al. (2006) reported deficits in spatial working memory in 4 to 5-month-old 5XFAD mice as reflected by reduced alternation percentage in the $\mathrm{Y}$ maze. Due to that, spatial working memory was analyzed to test whether the previously published impairment in the Y-maze task (Oakley et al., 2006) is still detectable in mice kept on the new genetic background. For this purpose, 5XFAD mice were tested in the cross-maze test in the first project (Chapter 2). Moreover, 5XFAD mice were compared to wildtype and other transgenic littermates in the second project (Chapter 3). In agreement with the previous results, the 5XFAD mouse model showed an age-dependent reduction in the spatial alternation percentage in the cross-maze starting from 6 months of age. It is noteworthy to mention that 
this reduction was not due to a decreased motor activity because 5XFAD mice showed comparable arm entries to their wildtype littermates. On the other hand, no change in the spatial alternation could be detected when 6-month-old 5XFAD mice were tested in the Ymaze. The only difference between the two tests lies in the higher level of complexity of the cross-maze because it has four arms instead of the three arms in the Y-maze. This makes it more challenging and thus more sensitive in detecting memory impairments. The different genetic backgrounds may underlie the discrepancy in the Y-maze performance between the current work and the work of Oakley et al. (2006). Genetic background has been reported to influence the performance of inbred wildtype mice (Holmes et al., 2002) and AD mouse models (Lassalle et al., 2008; Glazner et al., 2010). These differences in behavior might be due to genetic influences on neural development and thus on neuroanatomical, neurochemical, cellular, and molecular substrates associated with behavior.

Several AD models presented decreased spatial alternation levels with age, such as the Tg2576 (Ognibene et al., 2005) and the TASTPM (Howlett et al., 2004) mouse models. Other mouse models, such as the C3-3, E1-2 (Savonenko et al., 2003) and the APPswe + PS1/DeltaE9 (Lalonde et al., 2004), lacked this deficit.

Although no neuron loss could be detected in the hippocampus of the 5XFAD mice that may account for their spatial working memory impairment, Kimura and Ohno (2009) reported hippocampal CA1 synaptic failures in the form of basal synaptic transmission and LTP reduction at 6 months of age but not at 4 months of age. This LTP reduction correlated with reduced hippocampus-dependent formation of contextual fear memory. In agreement, the level of PSD-95, a major postsynaptic scaffold protein, was markedly decreased in the apical dendrites with a prominent immunoreactivity in neuronal soma in CA1 neurons of 6-monthold 5XFAD mice compared to 3-month-old 5XFAD mice (Shao et al., 2011). These findings may in part explain the reduced spatial alternation seen in the 5XFAD mice at 6 months of age and not at 3 months of age.

In summary, the current work recapitulates the spatial working memory impairment originally reported in 5XFAD mice, although only using a more sensitive test. 


\subsubsection{Reduced anxiety in 5XFAD mice}

In addition to cognitive impairment, a wide range of noncognitive neuropsychiatric changes are observed in AD. One of these symptoms is disinhibition, which is present in 25$30 \%$ of patients (Cummings, 2000). Disinhibition is manifested by socially unacceptable behavior and inappropriate euphoria (Chung and Cummings, 2000). The elevated plus maze measures the anxiety level in the mice. More time spent in the open arms reflects reduced anxiety and disinhibition, whereas less time spent in the open arms is an indication of increased anxiety levels. AD mouse models showed inconsistent anxiety tendencies. Some AD mouse models such Tg2576 (Ognibene et al., 2005), TASTPM (Pugh et al., 2007), PS1A246E (Lalonde et al., 2003a) and APP/PS1KI (Faure et al., 2011) mice displayed reduced anxiety, whereas other AD mouse models, such as the APP23 model did not demonstrate any change in its anxiety levels (Lalonde et al., 2002). On the other hand, other mouse models showed increased anxiety as revealed by enhanced innate and conditioned fear symptoms such as the $3 \times \mathrm{xTg}$ model. Elevated anxiety was claimed to correlate with the presence of intraneuronal $A \beta$ in the amygdala (Espana et al., 2009). When the 5XFAD mice were tested in the elevated plus maze, they showed reduced anxiety as reflected by increased time spent in the open arms compared to their wildtype littermates. This phenotype was first detected at 6 months of age. Interestingly, a very recent study showed that treatment of the 5XFAD mice with an antibody against low molecular weight $\mathrm{A} \beta_{\mathrm{pE3}-\mathrm{x}}$ oligomers from 4.5 months to 6 months of age stabilized the decline in the anxiety to the point when the treatment started (4.5 months) (Wirths et al., 2010b). This finding sheds light on the effect of the low molecular weight $\mathrm{A} \beta_{\mathrm{pE} 3-\mathrm{x}}$ oligomers on the disinhibition phenotype seen in 5XFAD mice.

Analysis of anxiety, exploration and locomotor activity in the open field paradigm confirmed the reduction in anxiety detected using elevated plus maze. The 5XFAD mice spent more time in the central region of the maze compared with their wildtype littermates. In contrast, locomotor behavior did not seem to be affected since there was no difference detected in the speed or the traveled distance during the 5 minutes of testing. Changes in the locomotor activity in the open field have been reported in some AD mouse models. Certain mice exhibited increased locomotor activity, such as TgCRND8 mice (Walker et al., 2011), APPC100.V717F transgenic (Boon et al., 2010), Tg2576 (Deacon et al., 2009) and 3xTg 
(Pietropaolo et al., 2008). Other mouse models presented reduced activity, such as APP751SL (Le Cudennec et al., 2008). On the other hand, other models were reported to have no obvious phenotype in the open field such as APPswe + PS1/DeltaE9 (Lalonde et al., 2004).

In conclusion, the current findings demonstrate for the first time changes in the anxiety level and the disinhibition phenotype of the 5XFAD mice as detected in the elevated plus maze and confirmed using the open field task.

\subsubsection{Plaque pathology and pyroglutamate-modified A $\beta$ in 5XFAD mice}

As expected in an APP/PS1 bigenic AD model, the 5XFAD mouse model was reported to develop extracellular plaque deposition as early as 2 months of age. The plaques first appeared in the deep layers of the cortex, the subiculum and the hippocampus. With age, plaques spread to cover most of the brain regions (Oakley et al., 2006). However, ageassociated dynamic changes in plaque pathology were never quantified. Therefore, two pools of $\mathrm{A} \beta$ plaques, in the hippocampus and the cortex, were analyzed at 3,6 and 12 months of age. These brain regions were chosen because they are the first regions where the plaque pathology starts. Also, as indicated above, these regions show differential intraneuronal A $\beta$ accumulation and neuron loss despite the presence of plaques. Moreover, they are relevant to the pathological and behavioral changes seen in the 5 XFAD mice.

The area covered with $A \beta$ plaques detected with a pan $A \beta$ antibody showed significant and continuous increase with age in both the cortex and the hippocampus. In the 5XFAD mice, the Swedish mutation produces higher levels of the total A $\beta$, whereas, APP Florida and London, and PS1 M146L and L286V mutations increase the production of A $\beta_{\mathrm{x}-42}$ specifically. As a consequence, the 5XFAD mice develop plaque deposition as early as 2 months of age, which is considered an early time point in comparison to most of the other AD mouse models (Oakley et al., 2006). Increased $A \beta_{x-42}$ levels appear to cause enhanced aggregation, early deposition and promote the toxicity of A $\beta$ (Barrow and Zagorski, 1991; Iwatsubo et al., 1994; Pike et al., 1995).

In addition to pan $A \beta$ plaques, $A \beta_{\mathrm{pE} 3-\mathrm{x}}$ plaque deposition was assessed in the $5 \mathrm{XFAD}$ mice. $A \beta_{\mathrm{pE} 3-\mathrm{x}}$ is a posttranslationally-modified form of $\mathrm{A} \beta$ that starts with a lactam ring at the third amino acid position (glutamate). The importance of the $\mathrm{A} \beta_{\mathrm{pE} 3-\mathrm{x}}$ arises from its 
characteristics: higher aggregation propensity (He and Barrow, 1999; Schilling et al., 2006), stronger hydrophobicity (Kuo et al., 1997), and increased toxicity compared with unmodified $\mathrm{A} \beta$ (Russo et al., 2002). The 5XFAD mice show a steady, age-dependent increase in the area covered by $\mathrm{A} \beta_{\mathrm{pE} 3-\mathrm{x}}$ in the hippocampus and the cortex as early as 3 months of age.

The predominance of $\mathrm{A} \beta_{\mathrm{pE} 3-\mathrm{x}}$ peptides in $\mathrm{AD}$ and $\mathrm{DS}$ patients and its characteristics raised the question about different $A \beta$ isoforms in $A D$ mouse models. In the commonly used Tg2576 mouse model, truncated and modified A $\beta$ appears at 16 months of age and comprises only $5 \%$ of the total $\mathrm{A} \beta$ peptides, even at the age of 23 months (Kawarabayashi et al., 2001). The authors suggested the scarcity of modified $A \beta$ as one of the reasons underlying the lack of neurofibrillary pathology and neuronal loss in $\mathrm{Tg} 2576$ brain. $\mathrm{A} \beta_{\mathrm{pE} 3-\mathrm{x}}$ and other modified forms of A $\beta$ were absent in another mouse model, the APP23, until 22 months of age. The difference in the composition of $\mathrm{A} \beta$ pools between AD and the APP23 mouse model might account for the different plaque morphology and the high solubility of A $\beta$ peptides in APP23 mice (Kuo et al., 2001). On the other hand, a recent study by Schieb et al. (2011) could detect minor amounts of $\mathrm{A} \beta_{\mathrm{PE} 3-\mathrm{x}}$ starting at 15 months of age in the brains of APP23 mice brain. Besides, the authors reported the presence of $\mathrm{A} \beta_{\mathrm{pE} 3-\mathrm{x}}$ in the brains of 24-month-old APP51 mice, which is transgenic for human wild type APP and has an onset of plaque pathology between 12 and 15 month of age (Schieb et al., 2011). Also, the APP[V717I] London (APP-Ld) mouse model was reported recently to develop an age-dependent increase in $\mathrm{A} \beta_{\mathrm{pE} 3-\mathrm{x}}$ starting from 12 months of age; however, the ratio of $A \beta_{\mathrm{pE} 3-42}$ to $\mathrm{A} \beta_{\mathrm{x}-42}$ was relatively low even at 15 months of age (Tanghe et al., 2010). The discrepancy in the $A \beta$ isoforms between AD patients and mouse models might be due to the short life span of the mice compared to the decades available for $\mathrm{A} \beta$ post-translational modification in $\mathrm{AD}$.

The APP/PS1KI mouse model is a double transgenic model with four mutations in APP and Presenilin-1 (PS1) (Casas et al., 2004). It develops age-dependent plaque deposition and intracellular $A \beta$ accumulation including $A \beta_{\mathrm{pE} 3-\mathrm{x}}$ as early as 2 month of age (Christensen et al., 2008). The area covered with $\mathrm{A} \beta_{\mathrm{pE} 3}$ positive plaques increases from less than $1 \%$ at 2 months of age to around $2 \%$ at 6 months of age until it covers more than $4.5 \%$ of the cortex at 10 months of age (Wirths et al., 2010a). 
Despite the value of the $\mathrm{AD}$ mouse models in providing $\mathrm{A} \beta$ heterogeneity, which is relatively similar to that seen in $\mathrm{AD}$, this heterogeneity makes it difficult to distinguish the individual toxic peptides from those which might only co-precipitate and could potentially be less harmful. Therefore, it was necessary to generate a mouse model that exclusively develops $\mathrm{N}$-truncated $\mathrm{A} \beta$. The TBA mouse models express $A \beta_{(\mathrm{Q}) 3-42}$ starting with an $\mathrm{N}$-terminal glutamine $(Q)$ residue at position three of $A \beta$, which facilitates the conversion to $A \beta_{\mathrm{pE} 3}$. These mice exhibit abundant intraneuronal $\mathrm{A} \beta_{\mathrm{pE} 3-\mathrm{x}}$ associated with neuron loss, striking neurological impairment and growth retardation (Wirths et al., 2009; Alexandru et al., 2011). However, the severe neurological phenotype and the premature death hinder their use for cognitive or longitudinal studies.

In light of that, choosing the correct $\mathrm{AD}$ mouse model harboring $\mathrm{A} \beta$ isoforms relevant to the purpose of the research is a critical point. This is especially important for studies assessing $A \beta$ pathology or testing therapeutic interventions against specific $A \beta$ isoforms. With these considerations, the 5XFAD mouse model represents a suitable model to study $A \beta_{\mathrm{PE} 3-\mathrm{x}}$. The early deposition and the abundance of $\mathrm{A} \beta_{\mathrm{pE} 3-\mathrm{x}}$ in this mouse model facilitate its use to test genetic and therapeutic strategies against this $A \beta$ isoform. 


\subsubsection{Conclusions of project I}

Based on the results of the current work

1. The $5 X F A D$ mouse model was validated on the $\mathrm{C} 57 \mathrm{BL} / 6 \mathrm{~J}$ genetic background.

2. 5XFAD mice were shown for the first time to develop motor deficits.

3. The neurological and motor phenotype in the SXFAD mice correlated with axonal deficits in the spinal cord, which coincided with the presence of intraneuronal $A \beta$.

4. Neuron loss in the fifth cortical layer of the 5XFAD mice was quantified and confirmed. It was found to coincide with the presence of intraneuronal $\mathrm{A} \beta$.

5. The reduced spatial alternation previously described in the 5XFAD mouse model was recapitulated.

6. Reduced anxiety was illustrated for the first time in the 5XFAD mice.

7. The 5XFAD mouse model was verified as valuable model to study the $A \beta_{\mathrm{pE} 3-\mathrm{x}}$ isoform. 


\subsection{Project II: "Evaluating the role of glutaminyl cyclase and pyroglutamate-modified $A \beta$ in $A D$ via the 5XFAD/hQC and 5XFAD/QC- KO mouse models"}

Beside $A \beta$ peptides starting with aspartate as the first amino acid $\left(\mathrm{A} \beta_{1-\mathrm{x}}\right)$, analysis of $\mathrm{A} \beta$ peptides isolated from $\mathrm{AD}$ and $\mathrm{DS}$ deposits revealed several truncated and modified $\mathrm{A} \beta$ species (Masters et al., 1985b; Roher et al., 1993; Naslund et al., 1994; Saido et al., 1995). Among these species, the $A \beta_{\mathrm{pE3} \text {-x }}$ peptides represent a major fraction (Saido et al., 1996). The conversion of $A \beta_{1-\mathrm{x}}$ into $\mathrm{A} \beta_{\mathrm{pE} 3-\mathrm{x}}$ leads to altered biochemical characteristics pointing to changes in aggregation, stability (He and Barrow, 1999; Schilling et al., 2006) and toxicity (Russo et al., 2002). Compiling findings from in vitro (Cynis et al., 2008a) and in vivo (Schilling et al., 2008c) experiments have recently proven that QC enzyme catalyzes the formation of $\mathrm{A} \beta_{\mathrm{pE} 3-\mathrm{x}}$, making it a potential therapeutic target.

As shown in the previous project, the $5 \mathrm{XFAD}$ mouse model represents a suitable model to study $\mathrm{QC}$ and $\mathrm{A} \beta_{\mathrm{pE} 3-\mathrm{x}}$ due to abundant $\mathrm{A} \beta_{\mathrm{pE} 3-\mathrm{x}}$ formation and the aggressiveness of AD pathology. With these considerations, the objective of the current project was to investigate, on a genetic basis, the role of $\mathrm{QC}$ in an $\mathrm{AD}$ mouse model. Therefore, the pathological role of QC was studied by generating 5XFAD mice overexpressing hQC (5XFAD/hQC mice). More importantly, 5XFAD mice lacking QC (5XFAD/QC-KO mice) were generated to analyze the contribution of endogenous $\mathrm{QC}$ to $\mathrm{A} \beta_{\mathrm{pE} 3-\mathrm{x}}$ formation and thus $\mathrm{AD}$ pathology. The resultant mice were analyzed in terms of $\mathrm{A} \beta$ pathology and behavioral impairments.

\subsubsection{The 5XFAD/hQC mouse model}

In order to study the role of $\mathrm{QC}$ overexpression in the production of $\mathrm{A} \beta_{\mathrm{pE} 3-\mathrm{x}}$ and thus on the pathology of $\mathrm{AD}$, the 5XFAD mice were crossed with hQC mice to generate the 5XFAD/hQC bigenic mouse model. This model produces ectopic QC expression; however, it provides a profound understanding for the pathological consequences of QC increase on AD pathology. 
The choice of the 5XFAD mouse model was supported by the age-dependent analysis of $\mathrm{A} \beta_{\mathrm{pE} 3-\mathrm{x}}$. By 6 months of age, 5XFAD mice show abundant $\mathrm{A} \beta_{\mathrm{pE} 3-\mathrm{x}}$ plaques. On the other hand, the higher activity of hQC in comparison to the murine QC (mQC) prompted the use of hQC instead of mQC overexpressing mice (Seifert et al., 2009). The activity of QC was 10-40 times higher in the hQC mice than that in the wildtype controls. hQC mice showed no neurological or dysmorphological phenotype. Primary behavioral screening of hQC mice (from 3 to 21 months of age) using the SHIRPA protocol did not reveal neurological or behavioral abnormalities (Personal communication with Dr. Sigrid Graubner). After crossing the 5XFAD with hQC mice, all the resultant genotypes were analyzed at 6 months of age. This time point was chosen since by that time the 5XFAD mice harbor significant $\mathrm{A} \beta_{\mathrm{pE} 3-\mathrm{x}}$ peptides and behavioral impairments in which modulation of QC activity may result in detectable effects.

To prove the expression of hQC transgene in 5XFAD/hQC and hQC mice, immunohistochemistry with an antibody detecting hQC was performed. The staining pattern of the transgene followed the Thy-1 promoter expression pattern where it covered different brain regions such as the cortex, hippocampus and midbrain regions. In general, QC staining showed perikaryal and axonal staining patterns, indicating transportation towards axon terminals. Endogenous mQC mRNA was reported to show ubiquitous distribution all over the body with the highest expression in the brain in the thalamus and the hippocampus followed by the cortex and the cerebellum (Cynis et al., 2008b).

In order to investigate the effect of $\mathrm{QC}$ on $\mathrm{A} \beta_{\mathrm{pE} 3-\mathrm{x}}$ production, it was crucial to assess the colocalization of $\mathrm{QC}$ with the $\mathrm{A} \beta_{\mathrm{pE} 3-\mathrm{x}}$ precursor, APP. For this purpose, double immunofluorescence staining was performed, revealing colocalization between APP and hQC in various brain regions, as both transgenes are under the control of Thy-1 promoter. On the subcellular level, both APP and hQC showed partial colocalization in the same cellular compartments. APP markedly labeled dystrophic neurites around plaques where it showed abundant colocalization with hQC. It is noteworthy to mention that APP is transported across the axons and accumulates in axonal swellings. Therefore, it is used for the detection of axonal pathology in traumatic brain injury (Dolinak and Reichard, 2006) and has been used to study axonopathy in the APP/PSKI mice (Wirths et al., 2007). Thus, the colocalization of both 
proteins provides another indication that $\mathrm{hQC}$ is also axonally transported. The current findings are consistent with a study by Cynis and colleagues who noted colocalization between APP and QC in the Golgi complex of HEK293 cotransfected cells (Cynis et al., 2008a). The authors suggested that this colocalization functions to catalyze the formation $\mathrm{A} \beta_{\mathrm{pE} 3-\mathrm{x}}$ seen in these cells.

Hartlage-Rubsamen et al. have noted, using immunohistochemistry, that QC expression was restricted to neurons whereas no QC signal could be detected in astrocytes from wildtype mice (Hartlage-Rubsamen et al., 2009). On the contrary, a recent study revealed QC activity in astrocytes culture media which was abolished in cells from QC-KO mice (Schilling et al., 2011). Moreover, using reversed phase HPLC coupled with ultraviolet detection, an increase in the QC activity in was illustrated in macrophages following stimulation with lipopolysaccharides (Chikuma et al., 2004). The discrepancy seen in the detection of QC expression may either be due to low QC levels in the basal conditions or due to the different sensitivities of the used techniques.

\subsubsection{1 hQC overexpression alters pyroglutamate-modified A $\mathrm{\beta}$ levels and plaque pathology}

The effect of hQC overexpression on $A \beta$ levels and plaque pathology was assessed in 6-month-old 5XFAD and 5XFAD/hQC mice. Analysis of peptide levels of $A \beta_{x-42}$ using ELISA did not reveal any significant differences in the TBS, SDS and Formic levels of A $\beta_{x-42}$. As expected, high QC levels in 5XFAD/hQC mice resulted in pronounced effects on $A \beta_{\mathrm{pE3}-42}$ peptides. 5XFAD/hQC mice showed significant elevation in all of $\mathrm{A} \beta_{\mathrm{PE} 3-42}$ fractions in ELISA. The apparent discrepancies between $A \beta_{\mathrm{pE} 3-42}$ and $A \beta_{x-42}$ are likely because $A \beta_{x-42}$ peptides are 1000 times more abundant than $A \beta_{\mathrm{pE} 3-42}$ in the brain homogenates ( $\mu \mathrm{g}$ versus $\mathrm{ng} / \mathrm{g}$ wet weight). Therefore, it is unlikely that the hQC-dependent increase in $\mathrm{A} \beta_{\mathrm{PE} 3-42}$ is reflected by concomitant increase in $\mathrm{A} \beta_{\mathrm{x}-42}$ level in a stochiometric pattern. Besides, it is hypothesized that $\mathrm{A} \beta_{\mathrm{PE} 3-42}$ does not necessarily lead to increased aggregation of $\mathrm{A} \beta_{\mathrm{X}-42}$, which might be due a saturation effect. In line with our findings, incubation of synthetic $A \beta_{3-x}$ with recombinant $\mathrm{QC}$ resulted in the conversion into $\mathrm{A} \beta_{\mathrm{pE} 3-\mathrm{x}}$; a reaction that is favored under acidic $\mathrm{pH}$ conditions and blocked by the presence of a QC inhibitor (Schilling et al., 2004). Similarly, 
cotransfection of $\beta$-TC3 cells with truncated $A \beta_{(\mathrm{E}) 3-42}$ and hQC lead to a significant elevation in $\mathrm{A} \beta_{\mathrm{pE} 3-42}$. Also, cotransfection of HEK293 cells with a truncated APP transgene starting with glutamate and an hQC transgene increased $A \beta_{\mathrm{pE} 3-42}$ levels (Cynis et al., 2008a). In both cases, addition of $Q C$ inhibitor reduced $A \beta_{\mathrm{pE} 3-42}$ levels with no impact on $\mathrm{A} \beta_{\mathrm{x}-42}$ peptides.

The consequences of hQC overexpression on the plaque pathology in 5XFAD mice were evaluated by quantifying the plaque load in the cortex. Consistent with the ELISA, $\mathrm{A} \beta_{\mathrm{pE} 3-42}$ plaque load was significantly elevated in $5 \mathrm{XFAD} / \mathrm{hQC}$ mice in comparison to agematched 5XFAD mice. While no significant increase in $A \beta_{x-42}$ levels could be depicted in the ELISA, plaque load of pan $A \beta$ was substantially increased in the cortex of $5 \mathrm{XFAD} / \mathrm{hQC}$ mice. $\mathrm{A} \beta_{\mathrm{pE} 3-\mathrm{x}}$ is known to possess higher aggregation propensity, oligomerization and enhanced $\beta$ sheet formation (He and Barrow, 1999). Therefore, it was previously suggested to have a seeding effect on plaques (Schilling et al., 2008c). However, the lack of an effect on the SDS and formic acid fractions of $A \beta_{x-42}$ in the ELISA contradicts the increased pan $A \beta$ plaque load in the cortex of $5 \mathrm{XFAD} / \mathrm{hQC}$ mice. This might be due to the fact that plaque load quantification was limited to the cortex, whereas the ELISA was performed on whole brain lysates. Thus, higher total $\mathrm{A} \beta$ plaque load in the cortex does not necessarily reflect an overall increase in brain $\mathrm{A} \beta$ plaques.

\subsubsection{2 hQC overexpression worsens the behavior impairments of 5XFAD mice}

The consequences of $\mathrm{QC}$ overexpression and the high $\mathrm{A} \beta_{\mathrm{PE} 3-42}$ levels in the $5 \mathrm{XFAD} / \mathrm{hQC}$ model were studied by following the behavior performance of these mice. As described earlier (Chapter 4.1.1), 5XFAD mice display motor impairments in the string suspension and the balance beam task starting from 9 months of age. Overexpression of hQC in $5 \mathrm{XFAD} / \mathrm{hQC}$ mice led to an earlier onset of the motor deficits already at 6 months of age. Although the 5XFAD mice showed normal alternation behavior in the Y-maze, 5XFAD/hQC mice had significantly reduced spatial alternation. In addition, the existing working memory deficits demonstrated by the 5XFAD mice in the cross maze were significantly exacerbated in the $5 \mathrm{XFAD} / \mathrm{hQC}$ mice. In both mazes, the reduced alternation was not due to reduced locomotor activity since the number of arm entries was not significantly changed. 
In agreement with the neurological phenotype seen in the $5 \mathrm{XFAD} / \mathrm{hQC}$ model, the TBA2 mouse model expressed tremendous amounts of intraneuronal $A \beta_{\mathrm{pE} 3-42}$ associated with loss of Purkinje cells, cerebellar atrophy, growth retardation and premature death. It also showed loss of motor coordination and ataxia (Wirths et al., 2009). Two recent transgenic models, TBA2.1 and TBA2.2, were developed expressing a similar transgene construct to that used in the TBA2 model but with milder pathology. In agreement, these mice displayed progressive $A \beta_{\mathrm{pE} 3-\mathrm{x}}$ deposits in brain regions with neuron loss, impaired LTP, microglial activation, and astrocytosis. Along with that, the TBA2.1 mice showed reduced activity and body weight, tremor, rigor, abnormal posture in the SHIRPA screen and impaired performance in the rotarod test in comparison to wildtype controls. Onset of overt behavioral symptoms associated with the peak of $\mathrm{A} \beta_{\mathrm{pE} 3-\mathrm{x}}$ (Alexandru et al., 2011). However, the early, severe motor deficits hindered the testing of the TBA mice in cognitive tasks. These data clearly illustrate the toxic effect of $\mathrm{A} \beta_{\mathrm{pE} 3-\mathrm{x}}$ in vivo and support the findings in the 5XFAD/hQC mouse model. On the contrary, a study by Youssef et al. (2008) compared the effect of $\mathrm{A} \beta_{\mathrm{pE} 3-42}$ and $\mathrm{A} \beta_{1-42}$ after intracerebroventricle injection in wildtype mice. They reported that both $\mathrm{A} \beta_{\mathrm{pE} 3-42}$ and $\mathrm{A} \beta_{1-42}$ induced similar deficits in the alternation percentage in the Y-maze and the delayed memory acquisition in the Y-maze and the Morris water maze tests. However, their study investigated the transient consequence of a single injection of synthetic peptides. Oppositely, the 5XFAD/hQC mouse model reflects continuous overproduction of internally produced and processed peptides.

Likewise, in vitro experiments proposed $A \beta_{\mathrm{pE} 3-40}$ to be more toxic to neurons and astorcytes compared to $A \beta_{1-40}$ (Russo et al., 2001). Similarly, $A \beta$ mixtures with high $A \beta_{\mathrm{pE} 3-42}$ contents resulted in significant reduction in the survival levels of neuroblastoma cells compared to $A \beta_{1-42}$ (Piccini et al., 2005).

Collectively, the $5 \mathrm{XFAD} / \mathrm{hQC}$ mouse model represents a valuable tool to study the pathological effects of $\mathrm{QC}$ on the $\mathrm{A} \beta_{\mathrm{pE} 3-42}$ production and thus on $\mathrm{AD}$ pathology. Despite the fact that it is an artificial overexpression model, it is a model which brings the QC enzyme together with the $A \beta$ precursor, APP. As a result, levels of $A \beta_{\mathrm{pE} 3-42}$ peptides and $A \beta_{\mathrm{pE} 3-\mathrm{x}}$ plaques were significantly higher in the bigenic mice. This led to a neurological phenotype in $5 \mathrm{XFAD} / \mathrm{hQC}$ mice represented by accelerated, significant motor and spatial working memory 
impairments in comparison to 5XFAD mice, an observation which supports the previously suggested toxicity of $\mathrm{A} \beta_{\mathrm{pE} 3-\mathrm{x}}$ in vivo.

\subsubsection{The 5XFAD/QC-KO mouse model}

In order to study the effects of endogenous QC on the pathology of $\mathrm{AD}, 5 \mathrm{XFAD} / \mathrm{QC}$ KO mice were generated. This model represents an invaluable tool to study, on a genetic basis, the effects of QC depletion. Ablation of QC resulted in a dramatic drop in enzymatic activity in the brain of QC-KO mice. Complete QC ablation did not show effects on mouse fertility or weight. Also, QC-KO mice performed indistinguishably from wildtype mice in motor tasks, the fear conditioning task, general activity and ingestion behavior. The finding that complete QC knockout did not induce significant abnormalities in the mice encouraged the use of these mice to study $\mathrm{AD}$ or to test therapeutic windows for suppression of QC (Schilling et al., 2011).

\subsubsection{Glutaminyl cyclase ablation reduces $A \beta$ concentration and plaque pathology in 5XFAD mice}

The impacts of $\mathrm{QC}$ knock-out on the $\mathrm{A} \beta$ concentrations and plaque pathology in the 5XFAD/QC-KO mice were evaluated using ELISA and quantitative plaque load analysis. In agreement with the ability of hQC overexpression to increase $\mathrm{A} \beta_{\mathrm{pE} 3-42}$ levels, knocking out QC diminished the TBS soluble $\mathrm{A} \beta_{\mathrm{pE} 3-42}$ to undetectable level in the ELISA, with a concomitant decrease in the SDS and formic acid soluble $A \beta_{\mathrm{pE} 3-42}$. Surprisingly, this was associated with reduction in SDS and formic acid soluble $A \beta_{x-42}$ peptides which might be explained in light of the reduced seeding effect of $A \beta_{\mathrm{pE} 3-\mathrm{x}}$. In good agreement with the ELISA results, plaque load quantifications showed a significant reduction in total $A \beta$ plaque pathology in the cortex. The effect on $A \beta_{\mathrm{pE} 3-\mathrm{x}}$ was more prominent with more than a $70 \%$ reduction in the area covered by $\mathrm{A} \beta_{\mathrm{pE} 3-\mathrm{x}}$ positive plaques in the cortex of $5 \mathrm{XFAD} / \mathrm{QC}-\mathrm{KO}$ mice.

The effects of QC reduction, by QC inhibitor, on AD pathology were studied in vivo for the first time when $A \beta_{(E) 3-40}$ peptides where injected in rat cortex. $A \beta_{(E) 3-40}$ peptides induced an increase in $\mathrm{A} \beta_{\mathrm{pE} 3-40}$ concentration and the generation of $\mathrm{A} \beta_{\mathrm{pE} 3-\mathrm{x}}$ positive plaques 24 hours after injection utilizing the rat's endogenous QC. This was significantly decreased via 
injection with a QC inhibitor (Schilling et al., 2008b). Utilizing another model system to prove the role of $\mathrm{QC}$, transgenic Drosophila flies expressing $\mathrm{A} \beta_{(\mathrm{Q}) 3-42}$ were treated for 4 weeks with a $\mathrm{QC}$ inhibitor and showed reduced $\mathrm{A} \beta_{\mathrm{PE} 3-42}$ production (Schilling et al., 2008c). Despite the difference in the methodology, the effects of the QC inhibitor are principally in agreement with the observations from the $5 \mathrm{XFAD} / \mathrm{QC}-\mathrm{KO}$ mice.

However, $\mathrm{A} \beta_{\mathrm{pE} 3-42}$ levels were not completely reduced in $5 \mathrm{XFAD} / \mathrm{QC}-\mathrm{KO}$ mice; thereby shedding light on the QC isoenzymes that might be responsible for the formation of the $\mathrm{A} \beta_{\mathrm{pE} 3-\mathrm{x}}$ in $5 \mathrm{XFAD} / \mathrm{QC}-\mathrm{KO}$ mice. This is supported by a similar observation in primary neurons with homozygous QC-KO. In these cells, the cellular QC-like activity persisted whereas the medium activity was diminished (Schilling et al., 2011). In this regard, an isoenzyme for $\mathrm{QC}$, isoQC, was recently isolated and characterized in mice and human (Cynis et al., 2008a; Stephan et al., 2009).

To analyze a possible contribution of isoQC to the remaining QC-like activity in the 5XFAD/QC-KO mice, western blot using an antibody against isoQC was performed. The protein levels of isoQC were unchanged in different brain regions between wildtype controls and QC-KO mice. This observation demonstrates that the residual $\mathrm{A} \beta_{\mathrm{pE} 3-\mathrm{x}}$ in the $5 \mathrm{XFAD} / \mathrm{QC}-$ $\mathrm{KO}$ model is possibly mediated by isoQC activity. Recent data by Alexandru et al. (2011) showed a similar pattern in which genetic elimination of $\mathrm{QC}$, but not isoQC, resulted in around $40 \%$ reduction in $\mathrm{A} \beta_{\mathrm{PE} 3-\mathrm{x}}$ level in the TBA2.1 mice. However, the TBA2.1 mice express glutamine (instead of the naturally occurring glutamate) at the third position which favors spontaneous cyclization into pyroglutamate. Further work is required to understand the physiological activity of isoQC especially, since it is likely that QC and isoQC have complementary functions. This should be a critical issue for developing drugs that target $\mathrm{QC}$ activity in different diseases.

\subsubsection{Glutaminyl cyclase knock-out rescues the spatial working memory impairment in 5XFAD mice}

The effect of $\mathrm{QC}$ ablation on $\mathrm{A} \beta$ and the plaque pathology raised the question about its impact on the behavior deficits. For this purpose, 6-month-old 5XFAD/QC-KO mice were tested in the same behavior tasks as the $5 \mathrm{XFAD} / \mathrm{hQC}$ mice. The most relevant task in this 
regard was the cross maze because it was one of the tests in which the 5XFAD mice were impaired at 6 months of age, therefore, enabling the detection of possible beneficial effects of QC-KO. Consistently, the working memory deficit of the 5XFAD mice was rescued in the 5XFAD/QC-KO mice, showing an alternation percentage indistinguishable from wildtype littermates. Although no changes were detected in other tasks, they are important to corroborate that complete QC knockout per se (5XFAD/QC-KO and QC-KO mice) does not induce significant behavioral impairments.

A previous study by Schilling et al. (2008) showed that application of a QC inhibitor in vivo had impacts that paralleled the current findings. Oral administration of a QC inhibitor in two AD mouse models, the Tg2576 and TASD-41, reduced the concentrations of A $\beta_{\mathrm{pE} 3-42}$, $\mathrm{A} \beta_{\mathrm{x}-42}$ and $\mathrm{A} \beta_{\mathrm{x}-40}$. Consequently, this reduction was accompanied with alleviation in the plaque density and gliosis in addition to improvements in contextual fear memory and spatial memory. Notably, similar to our findings, QC inhibitor did not reduce TBS soluble $A \beta_{x-42}$ and $\mathrm{A} \beta_{\mathrm{x}-40}$ levels, ruling out an effect on $\mathrm{A} \beta$ production.

Based on what is mentioned above, the 5XFAD/QC-KO mouse model is a valuable model for studying the endogenous contribution of QC to the pathology of AD. Complete elimination of $\mathrm{QC}$ reduced $\mathrm{A} \beta_{\mathrm{pE} 3-42}$ and $\mathrm{A} \beta_{\mathrm{x}-42}$ concentrations, alleviated the plaque pathology and improved the memory impairment in an aggressive AD mouse model.

In addition to what is mentioned above, I contributed to a recent study by our group in which a novel monoclonal antibody (9D5) against low molecular weight $\mathrm{A} \beta_{\mathrm{pE} 3-\mathrm{x}}$ oligomers was generated (Wirths et al., 2010b). The toxicity of $\mathrm{A} \beta_{\mathrm{pE} 3-42}$ peptides on neuroblastoma cells was completely abolished by adding the 9D5 antibody to the media. 9D5 did not have any effect when applied with $A \beta_{1-42}$. Also, levels of $A \beta_{\mathrm{pE} 3-42}$ oligomers were significantly reduced in the plasma of $\mathrm{AD}$ patients by $46 \%$ as compared to healthy controls. Over the past

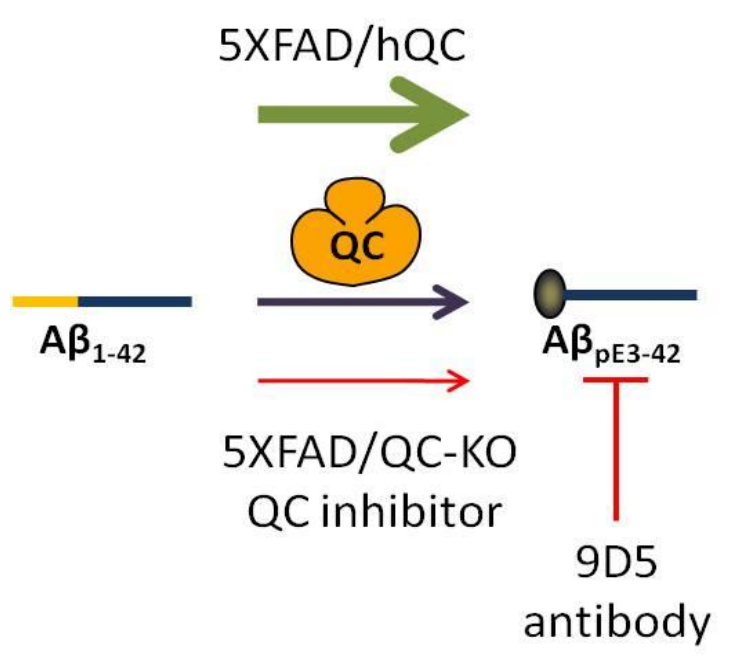

Fig. 12. Strategies for modulating $\mathbf{A} \boldsymbol{\beta}_{\mathrm{pE3}-\mathrm{x}}$ peptides by genetic modulation of $\mathrm{QC}$, treatment with $\mathrm{QC}$-inhibitor or by antibodies against $\mathbf{A} \boldsymbol{\beta}_{\text {pE3.-x. }}$ 
decade, immunotherapy gained increasing interest in the field of AD. Thus, immunotherapy against $A \beta_{\mathrm{PE} 3-\mathrm{x}}$ represents an interesting concept as well. In terms of therapeutic potential, passive immunization with 9D5 antibody in 4.5-month-old 5XFAD mice for 1.5 months was capable of reducing pan $A \beta$ plaque load and $A \beta_{\mathrm{PE} 3-\mathrm{x}}$ levels, and normalizing the anxiety changes seen at 6 months of age. Based on that, low molecular $\mathrm{A} \beta_{\mathrm{pE} 3-\mathrm{x}}$ oligomers represent a therapeutically and diagnostically effective target. These data, together with the findings from the 5XFAD/QC-KO model and QC-inhibitor studies, suggest that reduction of $\mathrm{A} \beta_{\mathrm{PE} 3-\mathrm{x}}$ levels as a beneficial approach for treating $\mathrm{AD}$ (Fig. 12).

In summary, findings from 5XFAD/hQC and 5XFAD/QC-KO mice clearly demonstrate on a genetic basis the major role played by $\mathrm{QC}$ in $\mathrm{AD}$ pathology by its direct effect on $\mathrm{A} \beta_{\mathrm{pE} 3-\mathrm{x}}$ production. It also presents $\mathrm{QC}$ as potential therapeutic target for $\mathrm{AD}$. 


\subsubsection{Conclusions of project II}

Based on the results of the current work

1. The role of $\mathrm{QC}$ in the pathology of $\mathrm{AD}$ was elucidated for the first time using genetic tools in an aggressive $\mathrm{AD}$ mouse model with abundant $\mathrm{A} \beta_{\mathrm{pE} 3-\mathrm{x}}$ formation.

2. Ectopic expression of hQC in the $5 \mathrm{XFAD} / \mathrm{hQC}$ model showed colocalization with $\mathrm{APP}$ and led to increased formation of $\mathrm{A} \beta_{\mathrm{pE} 3-42}$.

3. The increased $A \beta_{\mathrm{pE} 3-42}$ concentration aggravated the severity of the 5XFAD neurological phenotype.

4. Genetic elimination of endogenous $\mathrm{QC}$ in the $5 \mathrm{XFAD} / \mathrm{QC}-\mathrm{KO}$ reduced $\mathrm{A} \beta_{\mathrm{pE} 3-42}$ production and thus plaque pathology.

5. The residual $\mathrm{A} \beta_{\mathrm{pE} 3-42}$ in the $5 \mathrm{XFAD} / \mathrm{QC}-\mathrm{KO}$ suggests a role for isoQC enzymes.

6. Decreased $\mathrm{A} \beta_{\mathrm{PE} 3-42}$ alleviated the memory impairment in the 5XFAD mouse model. 


\section{Summary}

$\mathrm{AD}$ mouse models contributed widely to our understanding of the pathophysiology of $\mathrm{AD}$ and represent invaluable tools in testing potential therapeutic interventions. In the present study, age-dependent characterization of the neuropathological and behavioral hallmarks of the 5XFAD mouse model was performed, demonstrating that the 5XFAD mouse model develops a variety of $\mathrm{AD}$ neuropathological and behavioral hallmarks. It was shown for the first time that the 5XFAD mice develop neurological and motor phenotypes, which coincide with spinal cord pathology such axonopathy and intraneuronal $A \beta$. One of the most important characteristics originally reported in the 5XFAD mouse model is the neuron loss; however, this was never appropriately quantified. Using designed-based stereology, it was shown that the 5XFAD mice develop significant selective neuron loss in the fifth cortical of the frontal cortex which correlates with the accumulation of intraneuronal $\mathrm{A} \beta$ peptides. The 5XFAD mouse model develops early age-dependent alterations in anxiety levels and impaired spatial working memory starting from 6 months of age. Employing a variety of $A \beta$ specific antibodies, abundant intraneuronal $A \beta$ and extracellular plaque deposition was noted and showed age-dependent alterations. Altogether, the simultaneous occurrence of age-dependent neuropathological and the behavioral changes present the 5XFAD mouse model as an invaluable model to study AD. More importantly, the cosegregation of the mutations in the 5XFAD facilitates its use to generate bigenic mouse models.

$\mathrm{A} \beta_{\mathrm{PE} 3-\mathrm{x}}$ is a modified form of $\mathrm{A} \beta$ which is particularly interesting due to its predominance in $\mathrm{AD}$ brains and its high pathogenicity. The above mentioned considerations, in addition to the early deposition and the abundance of $A \beta_{\mathrm{pE} 3-\mathrm{x}}$ in the 5XFAD mouse model, prompted its use to test genetic strategies for modulating this $A \beta$ isoform. Several in vitro and in vivo lines of evidence suggested that $\mathrm{QC}$ enzyme is responsible for the formation of $\mathrm{A} \beta_{\mathrm{pE} 3-\mathrm{x}}$. In order to study the pathological contribution of QC to the pathology of AD, 5XFAD mice were crossed with hQC mice to generate a bigenic model of $\mathrm{AD}$ with $\mathrm{QC}$ overexpression, the $5 \mathrm{XFAD} / \mathrm{hQC}$ model. The $5 \mathrm{XFAD} / \mathrm{hQC}$ mouse model brought together $\mathrm{QC}$ and the $\mathrm{A} \beta$ precursor leading to higher $\mathrm{A} \beta_{\mathrm{PE} 3-\mathrm{x}}$ levels. As a consequence, the $5 \mathrm{XFAD} / \mathrm{hQC}$ mice exhibited higher $\mathrm{A} \beta_{\mathrm{pE} 3-\mathrm{x}}$ plaque pathology and accelerated motor and spatial working memory impairments in comparison to the 5XFAD mice. More importantly, the endogenous 
contribution of $\mathrm{QC}$ to $\mathrm{AD}$ pathology was studied by generating the 5XFAD/QC-KO bigenic mouse model. Complete depletion of $Q C$ reduced $A \beta_{\mathrm{PE} 3-42}$ and $A \beta_{x-42}$ concentrations, alleviated the plaque pathology and improved the memory impairment in the 5XFAD/QC-KO mouse model. Taken together, the outcome of this work clearly indicates the pivotal role of $\mathrm{QC}$ in AD pathology.

In summary, the aim of this thesis was to perform an age-dependent characterization of the 5XFAD mouse model and to verify its use in studying strategies that modulate $\mathrm{A} \beta_{\mathrm{pE} 3-\mathrm{x}}$ and thus $\mathrm{AD}$ pathology. Based on that, data from the $5 \mathrm{XFAD} / \mathrm{hQC}$ and the $5 \mathrm{XFAD} / \mathrm{QC}-\mathrm{KO}$ mice clearly demonstrate that $\mathrm{QC}$ is an essential enzyme modulating $\mathrm{A} \beta_{\mathrm{PE} 3-42}$ levels in vivo and proves on a genetic basis the concept that reduction of QC activity is an interesting new therapeutic approach for AD. 


\section{References}

Aggarwal NT, Wilson RS, Beck TL, Bienias JL, Bennett DA (2006) Motor dysfunction in mild cognitive impairment and the risk of incident Alzheimer disease. Arch Neurol 63:1763-1769.

Aletrino MA, Vogels OJ, Van Domburg PH, Ten Donkelaar HJ (1992) Cell loss in the nucleus raphes dorsalis in Alzheimer's disease. Neurobiol Aging 13:461-468.

Alexandru A, Jagla W, Graubner S, Becker A, Bauscher C, Kohlmann S, Sedlmeier R, Raber KA, Cynis H, Ronicke R, Reymann KG, Petrasch-Parwez E, Hartlage-Rubsamen M, Waniek A, Rossner S, Schilling S, Osmand AP, Demuth HU, von Horsten S (2011) Selective Hippocampal Neurodegeneration in Transgenic Mice Expressing Small Amounts of Truncated Abeta Is Induced by Pyroglutamate-Abeta Formation. J Neurosci 31:12790-12801.

Alonso A, Zaidi T, Novak M, Grundke-Iqbal I, Iqbal K (2001) Hyperphosphorylation induces self-assembly of tau into tangles of paired helical filaments/straight filaments. Proc Natl Acad Sci U S A 98:6923-6928.

Anderson CT, Sheets PL, Kiritani T, Shepherd GM (2010) Sublayer-specific microcircuits of corticospinal and corticostriatal neurons in motor cortex. Nat Neurosci 13:739-744.

Arendash GW, King DL, Gordon MN, Morgan D, Hatcher JM, Hope CE, Diamond DM (2001a) Progressive, age-related behavioral impairments in transgenic mice carrying both mutant amyloid precursor protein and presenilin-1 transgenes. Brain Res 891:4253.

Arendash GW, Gordon MN, Diamond DM, Austin LA, Hatcher JM, Jantzen P, DiCarlo G, Wilcock D, Morgan D (2001b) Behavioral assessment of Alzheimer's transgenic mice following long-term Abeta vaccination: task specificity and correlations between Abeta deposition and spatial memory. DNA Cell Biol 20:737-744.

Areosa SA, Sherriff F, McShane R (2005) Memantine for dementia. Cochrane Database Syst Rev:CD003154.

Ballard C, Waite J (2006) The effectiveness of atypical antipsychotics for the treatment of aggression and psychosis in Alzheimer's disease. Cochrane Database Syst Rev:CD003476.

Barrow CJ, Zagorski MG (1991) Solution structures of beta peptide and its constituent fragments: relation to amyloid deposition. Science 253:179-182.

Baumann K, Mandelkow EM, Biernat J, Piwnica-Worms H, Mandelkow E (1993) Abnormal Alzheimer-like phosphorylation of tau-protein by cyclin-dependent kinases cdk2 and cdk5. FEBS Lett 336:417-424.

Bayer TA, Wirths O (2011) Intraneuronal Abeta as a trigger for neuron loss: can this be translated into human pathology? Biochem Soc Trans 39:857-861.

Bayer TA, Cappai R, Masters CL, Beyreuther K, Multhaup G (1999) It all sticks together--the APP-related family of proteins and Alzheimer's disease. Mol Psychiatry 4:524-528.

Bekris LM, Yu CE, Bird TD, Tsuang DW (2010) Genetics of Alzheimer disease. J Geriatr Psychiatry Neurol 23:213-227.

Bertram L, Lill CM, Tanzi RE (2010) The genetics of Alzheimer disease: back to the future. Neuron 68:270-281. 
Blennow K, Bogdanovic N, Alafuzoff I, Ekman R, Davidsson P (1996) Synaptic pathology in Alzheimer's disease: relation to severity of dementia, but not to senile plaques, neurofibrillary tangles, or the ApoE4 allele. J Neural Transm 103:603-618.

Boon WC, van den Buuse M, Wegener N, Martin S, Chua HK, Bush AI, Masters CL, Adlard PA, Li QX (2010) Behavioural phenotype of APPC100.V717F transgenic mice overexpressing a mutant Abeta-bearing fragment is associated with reduced NMDA receptor density. Behav Brain Res 209:27-35.

Bottino CM, Castro CC, Gomes RL, Buchpiguel CA, Marchetti RL, Neto MR (2002) Volumetric MRI measurements can differentiate Alzheimer's disease, mild cognitive impairment, and normal aging. Int Psychogeriatr 14:59-72.

Braak H, Braak E, Yilmazer D, de Vos RA, Jansen EN, Bohl J (1996) Pattern of brain destruction in Parkinson's and Alzheimer's diseases. J Neural Transm 103:455-490.

Breyhan H, Wirths O, Duan K, Marcello A, Rettig J, Bayer TA (2009) APP/PS1KI bigenic mice develop early synaptic deficits and hippocampus atrophy. Acta Neuropathol 117:677-685.

Brouwers N, Sleegers K, Van Broeckhoven C (2008) Molecular genetics of Alzheimer's disease: an update. Ann Med 40:562-583.

Bugiani O, Giaccone G, Frangione B, Ghetti B, Tagliavini F (1989) Alzheimer patients: preamyloid deposits are more widely distributed than senile plaques throughout the central nervous system. Neurosci Lett 103:263-268.

Bullock R, Dengiz A (2005) Cognitive performance in patients with Alzheimer's disease receiving cholinesterase inhibitors for up to 5 years. Int J Clin Pract 59:817-822.

Bullock R, Touchon J, Bergman H, Gambina G, He Y, Rapatz G, Nagel J, Lane R (2005) Rivastigmine and donepezil treatment in moderate to moderately-severe Alzheimer's disease over a 2-year period. Curr Med Res Opin 21:1317-1327.

Burns A, Jacoby R, Levy R (1990) Psychiatric phenomena in Alzheimer's disease. III: Disorders of mood. Br J Psychiatry 157:81-86, 92-84.

Busby WH, Jr., Quackenbush GE, Humm J, Youngblood WW, Kizer JS (1987) An enzyme(s) that converts glutaminyl-peptides into pyroglutamyl-peptides. Presence in pituitary, brain, adrenal medulla, and lymphocytes. J Biol Chem 262:8532-8536.

Busch C, Bohl J, Ohm TG (1997) Spatial, temporal and numeric analysis of Alzheimer changes in the nucleus coeruleus. Neurobiol Aging 18:401-406.

Bussiere T, Giannakopoulos P, Bouras C, Perl DP, Morrison JH, Hof PR (2003) Progressive degeneration of nonphosphorylated neurofilament protein-enriched pyramidal neurons predicts cognitive impairment in Alzheimer's disease: stereologic analysis of prefrontal cortex area 9. J Comp Neurol 463:281-302.

Buxbaum JD, Liu KN, Luo Y, Slack JL, Stocking KL, Peschon JJ, Johnson RS, Castner BJ, Cerretti DP, Black RA (1998) Evidence that tumor necrosis factor alpha converting enzyme is involved in regulated alpha-secretase cleavage of the Alzheimer amyloid protein precursor. J Biol Chem 273:27765-27767.

Cai H, Wang Y, McCarthy D, Wen H, Borchelt DR, Price DL, Wong PC (2001) BACE1 is the major beta-secretase for generation of Abeta peptides by neurons. Nat Neurosci 4:233-234. 
Calhoun ME, Wiederhold KH, Abramowski D, Phinney AL, Probst A, Sturchler-Pierrat C, Staufenbiel M, Sommer B, Jucker M (1998) Neuron loss in APP transgenic mice. Nature 395:755-756.

Calingasan NY, Chen J, Kiaei M, Beal MF (2005) Beta-amyloid 42 accumulation in the lumbar spinal cord motor neurons of amyotrophic lateral sclerosis patients. Neurobiol Dis 19:340-347.

Carlson GA, Borchelt DR, Dake A, Turner S, Danielson V, Coffin JD, Eckman C, Meiners J, Nilsen SP, Younkin SG, Hsiao KK (1997) Genetic modification of the phenotypes produced by amyloid precursor protein overexpression in transgenic mice. Hum Mol Genet 6:1951-1959.

Casas C, Sergeant N, Itier JM, Blanchard V, Wirths O, van der Kolk N, Vingtdeux V, van de Steeg E, Ret G, Canton T, Drobecq H, Clark A, Bonici B, Delacourte A, Benavides J, Schmitz C, Tremp G, Bayer TA, Benoit P, Pradier L (2004) Massive CA1/2 neuronal loss with intraneuronal and N-terminal truncated Abeta42 accumulation in a novel Alzheimer transgenic model. Am J Pathol 165:1289-1300.

Caspersen C, Wang N, Yao J, Sosunov A, Chen X, Lustbader JW, Xu HW, Stern D, McKhann G, Yan SD (2005) Mitochondrial Abeta: a potential focal point for neuronal metabolic dysfunction in Alzheimer's disease. Faseb J 19:2040-2041.

Castellano JM, Kim J, Stewart FR, Jiang H, DeMattos RB, Patterson BW, Fagan AM, Morris JC, Mawuenyega KG, Cruchaga C, Goate AM, Bales KR, Paul SM, Bateman RJ, Holtzman DM (2011) Human apoE isoforms differentially regulate brain amyloid-beta peptide clearance. Sci Transl Med 3:89ra57.

Chambers JK, Kuribayashi H, Ikeda S, Une Y (2010) Distribution of neprilysin and deposit patterns of Abeta subtypes in the brains of aged squirrel monkeys (Saimiri sciureus). Amyloid 17:75-82.

Chen H, Epelbaum S, Delatour B (2011) Fiber Tracts Anomalies in APPxPS1 Transgenic Mice Modeling Alzheimer's Disease. J Aging Res 2011:281274.

Chevalier-Larsen E, Holzbaur EL (2006) Axonal transport and neurodegenerative disease. Biochim Biophys Acta 1762:1094-1108.

Chikuma T, Taguchi K, Yamaguchi M, Hojo H, Kato T (2004) Improved determination of bovine glutaminyl cyclase activity using precolumn derivatization and reversed-phase high-performance liquid chromatography with ultraviolet detection. J Chromatogr B Analyt Technol Biomed Life Sci 806:113-118.

Chishti MA, Yang DS, Janus C, Phinney AL, Horne P, Pearson J, Strome R, Zuker N, Loukides J, French J, Turner S, Lozza G, Grilli M, Kunicki S, Morissette C, Paquette J, Gervais F, Bergeron C, Fraser PE, Carlson GA, George-Hyslop PS, Westaway D (2001) Early-onset amyloid deposition and cognitive deficits in transgenic mice expressing a double mutant form of amyloid precursor protein 695. J Biol Chem 276:21562-21570.

Chow VW, Mattson MP, Wong PC, Gleichmann M (2011) An overview of APP processing enzymes and products. Neuromolecular Med 12:1-12.

Christensen DZ, Kraus SL, Flohr A, Cotel MC, Wirths O, Bayer TA (2008) Transient intraneuronal Abeta rather than extracellular plaque pathology correlates with neuron loss in the frontal cortex of APP/PS1KI mice. Acta Neuropathol 116:647-655. 
Chui DH, Dobo E, Makifuchi T, Akiyama H, Kawakatsu S, Petit A, Checler F, Araki W, Takahashi K, Tabira T (2001) Apoptotic neurons in Alzheimer's disease frequently show intracellular Abeta42 labeling. J Alzheimers Dis 3:231-239.

Chui HC, Lyness SA, Sobel E, Schneider LS (1994) Extrapyramidal signs and psychiatric symptoms predict faster cognitive decline in Alzheimer's disease. Arch Neurol 51:676681.

Chung JA, Cummings JL (2000) Neurobehavioral and neuropsychiatric symptoms in Alzheimer's disease: characteristics and treatment. Neurol Clin 18:829-846.

Citron M, Teplow DB, Selkoe DJ (1995) Generation of amyloid beta protein from its precursor is sequence specific. Neuron 14:661-670.

Citron M, Oltersdorf T, Haass C, McConlogue L, Hung AY, Seubert P, Vigo-Pelfrey C, Lieberburg I, Selkoe DJ (1992) Mutation of the beta-amyloid precursor protein in familial Alzheimer's disease increases beta-protein production. Nature 360:672-674.

Coma M, Sereno L, Da Rocha-Souto B, Scotton TC, Espana J, Sanchez MB, Rodriguez M, Agullo J, Guardia-Laguarta C, Garcia-Alloza M, Borrelli LA, Clarimon J, Lleo A, Bacskai BJ, Saura CA, Hyman BT, Gomez-Isla T (2010) Triflusal reduces dense-core plaque load, associated axonal alterations and inflammatory changes, and rescues cognition in a transgenic mouse model of Alzheimer's disease. Neurobiol Dis 38:482491.

Cook DG, Forman MS, Sung JC, Leight S, Kolson DL, Iwatsubo T, Lee VM, Doms RW (1997) Alzheimer's A beta(1-42) is generated in the endoplasmic reticulum/intermediate compartment of NT2N cells. Nat Med 3:1021-1023.

Cras P, Kawai M, Lowery D, Gonzalez-DeWhitt P, Greenberg B, Perry G (1991) Senile plaque neurites in Alzheimer disease accumulate amyloid precursor protein. Proc Natl Acad Sci U S A 88:7552-7556.

Cras P, van Harskamp F, Hendriks L, Ceuterick C, van Duijn CM, Stefanko SZ, Hofman A, Kros JM, Van Broeckhoven C, Martin JJ (1998) Presenile Alzheimer dementia characterized by amyloid angiopathy and large amyloid core type senile plaques in the APP 692Ala-->Gly mutation. Acta Neuropathol 96:253-260.

Cullen KM, Halliday GM, Double KL, Brooks WS, Creasey H, Broe GA (1997) Cell loss in the nucleus basalis is related to regional cortical atrophy in Alzheimer's disease. Neuroscience 78:641-652.

Cummings JL (2000) Cognitive and behavioral heterogeneity in Alzheimer's disease: seeking the neurobiological basis. Neurobiol Aging 21:845-861.

Cupers P, Bentahir M, Craessaerts K, Orlans I, Vanderstichele H, Saftig P, De Strooper B, Annaert W (2001) The discrepancy between presenilin subcellular localization and gamma-secretase processing of amyloid precursor protein. J Cell Biol 154:731-740.

Cynis H, Scheel E, Saido TC, Schilling S, Demuth HU (2008a) Amyloidogenic processing of amyloid precursor protein: evidence of a pivotal role of glutaminyl cyclase in generation of pyroglutamate-modified amyloid-beta. Biochemistry 47:7405-7413.

Cynis H, Schilling S, Bodnar M, Hoffmann T, Heiser U, Saido TC, Demuth HU (2006) Inhibition of glutaminyl cyclase alters pyroglutamate formation in mammalian cells. Biochim Biophys Acta 1764:1618-1625.

Cynis H, Rahfeld JU, Stephan A, Kehlen A, Koch B, Wermann M, Demuth HU, Schilling S (2008b) Isolation of an isoenzyme of human glutaminyl cyclase: retention in the Golgi 
complex suggests involvement in the protein maturation machinery. $\mathrm{J}$ Mol Biol 379:966-980.

D'Andrea MR, Nagele RG, Wang HY, Lee DH (2002) Consistent immunohistochemical detection of intracellular beta-amyloid42 in pyramidal neurons of Alzheimer's disease entorhinal cortex. Neurosci Lett 333:163-166.

Dahl SW, Slaughter C, Lauritzen C, Bateman RC, Jr., Connerton I, Pedersen J (2000) Carica papaya glutamine cyclotransferase belongs to a novel plant enzyme subfamily: cloning and characterization of the recombinant enzyme. Protein Expr Purif 20:27-36.

Dahm R (2006) Alzheimer's discovery. Curr Biol 16:R906-910.

Davies CA, Mann DM, Sumpter PQ, Yates PO (1987) A quantitative morphometric analysis of the neuronal and synaptic content of the frontal and temporal cortex in patients with Alzheimer's disease. J Neurol Sci 78:151-164.

Davies P, Maloney AJ (1976) Selective loss of central cholinergic neurons in Alzheimer's disease. Lancet 2:1403.

Dawson GR, Seabrook GR, Zheng H, Smith DW, Graham S, O'Dowd G, Bowery BJ, Boyce S, Trumbauer ME, Chen HY, Van der Ploeg LH, Sirinathsinghji DJ (1999) Agerelated cognitive deficits, impaired long-term potentiation and reduction in synaptic marker density in mice lacking the beta-amyloid precursor protein. Neuroscience 90:113.

De Vos KJ, Grierson AJ, Ackerley S, Miller CC (2008) Role of axonal transport in neurodegenerative diseases. Annu Rev Neurosci 31:151-173.

Deacon RM, Koros E, Bornemann KD, Rawlins JN (2009) Aged Tg2576 mice are impaired on social memory and open field habituation tests. Behav Brain Res 197:466-468.

DeKosky ST, Scheff SW (1990) Synapse loss in frontal cortex biopsies in Alzheimer's disease: correlation with cognitive severity. Ann Neurol 27:457-464.

Dember WN, Fowler H (1958) Spontaneous alternation behavior. Psychol Bull 55:412-428.

Devi L, Ohno M (2010) Genetic reductions of beta-site amyloid precursor protein-cleaving enzyme 1 and amyloid-beta ameliorate impairment of conditioned taste aversion memory in 5XFAD Alzheimer's disease model mice. Eur J Neurosci 31:110-118.

Devi L, Alldred MJ, Ginsberg SD, Ohno M (2010) Sex- and brain region-specific acceleration of beta-amyloidogenesis following behavioral stress in a mouse model of Alzheimer's disease. Mol Brain 3:34.

Dickson DW (1997) The pathogenesis of senile plaques. J Neuropathol Exp Neurol 56:321339.

Ditaranto K, Tekirian TL, Yang AJ (2001) Lysosomal membrane damage in soluble Abetamediated cell death in Alzheimer's disease. Neurobiol Dis 8:19-31.

Divac I (1975) Magnocellular nuclei of the basal forebrain project to neocortex, brain stem, and olfactory bulb. Review of some functional correlates. Brain Res 93:385-398.

Dodart JC, Mathis C, Saura J, Bales KR, Paul SM, Ungerer A (2000) Neuroanatomical abnormalities in behaviorally characterized APP(V717F) transgenic mice. Neurobiol Dis 7:71-85.

Dolinak D, Reichard R (2006) An overview of inflicted head injury in infants and young children, with a review of beta-amyloid precursor protein immunohistochemistry. Arch Pathol Lab Med 130:712-717. 
Drewes G, Lichtenberg-Kraag B, Doring F, Mandelkow EM, Biernat J, Goris J, Doree M, Mandelkow E (1992) Mitogen activated protein (MAP) kinase transforms tau protein into an Alzheimer-like state. Embo J 11:2131-2138.

Duff K, Eckman C, Zehr C, Yu X, Prada CM, Perez-tur J, Hutton M, Buee L, Harigaya Y, Yager D, Morgan D, Gordon MN, Holcomb L, Refolo L, Zenk B, Hardy J, Younkin S (1996) Increased amyloid-beta42(43) in brains of mice expressing mutant presenilin 1. Nature 383:710-713.

Duyckaerts C, Delatour B, Potier MC (2009) Classification and basic pathology of Alzheimer disease. Acta Neuropathol 118:5-36.

Eckman, C.B., Mehta, N.D., Crook, R., Perez-tur, J., Prihar, G., Pfeiffer, E., Graff-Radford, N., Hinder, P., Yager, D., Zenk, B., et al. (1997) A new pathogenic mutation in the APP gene (I716V) increases the relative proportion of A beta 42(43). Hum Mol Genet 6:2087-2089.

Espana J, Gimenez-Llort L, Valero J, Minano A, Rabano A, Rodriguez-Alvarez J, LaFerla FM, Saura CA (2009) Intraneuronal beta-amyloid accumulation in the amygdala enhances fear and anxiety in Alzheimer's disease transgenic mice. Biol Psychiatry 67:513-521.

Ewers M, Morgan DG, Gordon MN, Woodruff-Pak DS (2006) Associative and motor learning in 12-month-old transgenic APP+PS1 mice. Neurobiol Aging 27:1118-1128.

Farrer LA, Cupples LA, Haines JL, Hyman B, Kukull WA, Mayeux R, Myers RH, PericakVance MA, Risch N, van Duijn CM (1997) Effects of age, sex, and ethnicity on the association between apolipoprotein $\mathrm{E}$ genotype and Alzheimer disease. A metaanalysis. APOE and Alzheimer Disease Meta Analysis Consortium. Jama 278:13491356.

Faure A, Verret L, Bozon B, El Tannir El Tayara N, Ly M, Kober F, Dhenain M, Rampon C, Delatour B (2011) Impaired neurogenesis, neuronal loss, and brain functional deficits in the APPxPS1-Ki mouse model of Alzheimer's disease. Neurobiol Aging 32:407418.

Feng G, Mellor RH, Bernstein M, Keller-Peck C, Nguyen QT, Wallace M, Nerbonne JM, Lichtman JW, Sanes JR (2000) Imaging neuronal subsets in transgenic mice expressing multiple spectral variants of GFP. Neuron 28:41-51.

Ferri CP, Prince M, Brayne C, Brodaty H, Fratiglioni L, Ganguli M, Hall K, Hasegawa K, Hendrie H, Huang Y, Jorm A, Mathers C, Menezes PR, Rimmer E, Scazufca M (2005) Global prevalence of dementia: a Delphi consensus study. Lancet 366:2112-2117.

Fischer WH, Spiess J (1987) Identification of a mammalian glutaminyl cyclase converting glutaminyl into pyroglutamyl peptides. Proc Natl Acad Sci U S A 84:3628-3632.

Folstein MF, Folstein SE, McHugh PR (1975) "Mini-mental state". A practical method for grading the cognitive state of patients for the clinician. J Psychiatr Res 12:189-198.

Forstl H, Kurz A (1999) Clinical features of Alzheimer's disease. Eur Arch Psychiatry Clin Neurosci 249:288-290.

Games D, Adams D, Alessandrini R, Barbour R, Berthelette P, Blackwell C, Carr T, Clemens J, Donaldson T, Gillespie F, et al. (1995) Alzheimer-type neuropathology in transgenic mice overexpressing V717F beta-amyloid precursor protein. Nature 373:523-527.

Gandy S (2005) The role of cerebral amyloid beta accumulation in common forms of Alzheimer disease. J Clin Invest 115:1121-1129. 
Gauthier S, Reisberg B, Zaudig M, Petersen RC, Ritchie K, Broich K, Belleville S, Brodaty H, Bennett D, Chertkow H, Cummings JL, de Leon M, Feldman H, Ganguli M, Hampel H, Scheltens P, Tierney MC, Whitehouse P, Winblad B (2006) Mild cognitive impairment. Lancet 367:1262-1270.

Glazner KA, Odero GL, Anema E, Motnenko A, Schapansky J, Grossman D, Oliver DR, Glazner GW, Albensi BC (2010) Strain specific differences in memory and neuropathology in a mouse model of Alzheimer's disease. Life Sci 86:942-950.

Glenner GG, Wong CW (1984) Alzheimer's disease: initial report of the purification and characterization of a novel cerebrovascular amyloid protein. Biochem Biophys Res Commun 120:885-890.

Goate A, Chartier-Harlin MC, Mullan M, Brown J, Crawford F, Fidani L, Giuffra L, Haynes A, Irving N, James L, et al. (1991) Segregation of a missense mutation in the amyloid precursor protein gene with familial Alzheimer's disease. Nature 349:704-706.

Goedert M, Spillantini MG, Jakes R, Rutherford D, Crowther RA (1989) Multiple isoforms of human microtubule-associated protein tau: sequences and localization in neurofibrillary tangles of Alzheimer's disease. Neuron 3:519-526.

Gomez-Isla T, Price JL, McKeel DW, Jr., Morris JC, Growdon JH, Hyman BT (1996) Profound loss of layer II entorhinal cortex neurons occurs in very mild Alzheimer's disease. J Neurosci 16:4491-4500.

Goren HJ, Bauce LG, Vale W (1977) Forces and structural limitations of binding of thyrotrophin-releasing factor to the thyrotrophin-releasing receptor: the pyroglutamic acid moiety. Mol Pharmacol 13:606-614.

Gorevic PD, Goni F, Pons-Estel B, Alvarez F, Peress NS, Frangione B (1986) Isolation and partial characterization of neurofibrillary tangles and amyloid plaque core in Alzheimer's disease: immunohistological studies. J Neuropathol Exp Neurol 45:647664.

Gouras GK, Tampellini D, Takahashi RH, Capetillo-Zarate E (2010) Intraneuronal betaamyloid accumulation and synapse pathology in Alzheimer's disease. Acta Neuropathol 119:523-541.

Gouras GK, Tsai J, Naslund J, Vincent B, Edgar M, Checler F, Greenfield JP, Haroutunian V, Buxbaum JD, Xu H, Greengard P, Relkin NR (2000) Intraneuronal Abeta42 accumulation in human brain. Am J Pathol 156:15-20.

Greenfield JP, Tsai J, Gouras GK, Hai B, Thinakaran G, Checler F, Sisodia SS, Greengard P, $\mathrm{Xu} \mathrm{H}$ (1999) Endoplasmic reticulum and trans-Golgi network generate distinct populations of Alzheimer beta-amyloid peptides. Proc Natl Acad Sci U S A 96:742747.

Grundke-Iqbal I, Iqbal K, Tung YC, Quinlan M, Wisniewski HM, Binder LI (1986) Abnormal phosphorylation of the microtubule-associated protein tau (tau) in Alzheimer cytoskeletal pathology. Proc Natl Acad Sci U S A 83:4913-4917.

Grundke-Iqbal I, Iqbal K, George L, Tung YC, Kim KS, Wisniewski HM (1989) Amyloid protein and neurofibrillary tangles coexist in the same neuron in Alzheimer disease. Proc Natl Acad Sci U S A 86:2853-2857.

Gunn AP, Masters CL, Cherny RA (2010) Pyroglutamate-Abeta: Role in the natural history of Alzheimer's disease. Int J Biochem Cell Biol 42:1915-1918. 
Guo Q, Sebastian L, Sopher BL, Miller MW, Ware CB, Martin GM, Mattson MP (1999) Increased vulnerability of hippocampal neurons from presenilin-1 mutant knock-in mice to amyloid beta-peptide toxicity: central roles of superoxide production and caspase activation. J Neurochem 72:1019-1029.

Gyure KA, Durham R, Stewart WF, Smialek JE, Troncoso JC (2001) Intraneuronal abetaamyloid precedes development of amyloid plaques in Down syndrome. Arch Pathol Lab Med 125:489-492.

Haas C, Hung AY, Citron M, Teplow DB, Selkoe DJ (1995) beta-Amyloid, protein processing and Alzheimer's disease. Arzneimittelforschung 45:398-402.

Hardy J, Allsop D (1991) Amyloid deposition as the central event in the aetiology of Alzheimer's disease. Trends Pharmacol Sci 12:383-388.

Harigaya Y, Saido TC, Eckman CB, Prada CM, Shoji M, Younkin SG (2000) Amyloid beta protein starting pyroglutamate at position 3 is a major component of the amyloid deposits in the Alzheimer's disease brain. Biochem Biophys Res Commun 276:422427.

Hartig W, Goldhammer S, Bauer U, Wegner F, Wirths O, Bayer TA, Grosche J (2010) Concomitant detection of beta-amyloid peptides with $\mathrm{N}$-terminal truncation and different $\mathrm{C}$-terminal endings in cortical plaques from cases with Alzheimer's disease, senile monkeys and triple transgenic mice. J Chem Neuroanat 40:82-92.

Hartlage-Rubsamen M, Staffa K, Waniek A, Wermann M, Hoffmann T, Cynis H, Schilling S, Demuth HU, Rossner S (2009) Developmental expression and subcellular localization of glutaminyl cyclase in mouse brain. Int J Dev Neurosci 27:825-835.

Hartmann T, Bieger SC, Bruhl B, Tienari PJ, Ida N, Allsop D, Roberts GW, Masters CL, Dotti CG, Unsicker K, Beyreuther K (1997) Distinct sites of intracellular production for Alzheimer's disease Abeta40/42 amyloid peptides. Nat Med 3:1016-1020.

He W, Barrow CJ (1999) The A beta 3-pyroglutamyl and 11-pyroglutamyl peptides found in senile plaque have greater beta-sheet forming and aggregation propensities in vitro than full-length A beta. Biochemistry 38:10871-10877.

Herholz K, Salmon E, Perani D, Baron JC, Holthoff V, Frolich L, Schonknecht P, Ito K, Mielke R, Kalbe E, Zundorf G, Delbeuck X, Pelati O, Anchisi D, Fazio F, Kerrouche N, Desgranges B, Eustache F, Beuthien-Baumann B, Menzel C, Schroder J, Kato T, Arahata Y, Henze M, Heiss WD (2002) Discrimination between Alzheimer dementia and controls by automated analysis of multicenter FDG PET. Neuroimage 17:302-316.

Hirokawa N, Funakoshi T, Sato-Harada R, Kanai Y (1996) Selective stabilization of tau in axons and microtubule-associated protein $2 \mathrm{C}$ in cell bodies and dendrites contributes to polarized localization of cytoskeletal proteins in mature neurons. $\mathrm{J}$ Cell Biol 132:667-679.

Holcomb L, Gordon MN, McGowan E, Yu X, Benkovic S, Jantzen P, Wright K, Saad I, Mueller R, Morgan D, Sanders S, Zehr C, O'Campo K, Hardy J, Prada CM, Eckman C, Younkin S, Hsiao K, Duff K (1998) Accelerated Alzheimer-type phenotype in transgenic mice carrying both mutant amyloid precursor protein and presenilin 1 transgenes. Nat Med 4:97-100.

Holmes A, Wrenn CC, Harris AP, Thayer KE, Crawley JN (2002) Behavioral profiles of inbred strains on novel olfactory, spatial and emotional tests for reference memory in mice. Genes Brain Behav 1:55-69. 
Holtzman DM, Morris JC, Goate AM (2011) Alzheimer's disease: the challenge of the second century. Sci Transl Med 3:77sr71.

Hongpaisan J, Sun MK, Alkon DL (2011) PKC epsilon activation prevents synaptic loss, Abeta elevation, and cognitive deficits in Alzheimer's disease transgenic mice. $\mathrm{J}$ Neurosci 31:630-643.

Hosoda R, Saido TC, Otvos L, Jr., Arai T, Mann DM, Lee VM, Trojanowski JQ, Iwatsubo T (1998) Quantification of modified amyloid beta peptides in Alzheimer disease and Down syndrome brains. J Neuropathol Exp Neurol 57:1089-1095.

Howlett DR, Richardson JC, Austin A, Parsons AA, Bate ST, Davies DC, Gonzalez MI (2004) Cognitive correlates of Abeta deposition in male and female mice bearing amyloid precursor protein and presenilin-1 mutant transgenes. Brain Res 1017:130136.

Hsiao K, Chapman P, Nilsen S, Eckman C, Harigaya Y, Younkin S, Yang F, Cole G (1996) Correlative memory deficits, Abeta elevation, and amyloid plaques in transgenic mice. Science 274:99-102.

Huse JT, Liu K, Pijak DS, Carlin D, Lee VM, Doms RW (2002) Beta-secretase processing in the trans-Golgi network preferentially generates truncated amyloid species that accumulate in Alzheimer's disease brain. J Biol Chem 277:16278-16284.

Hussain I, Powell D, Howlett DR, Tew DG, Meek TD, Chapman C, Gloger IS, Murphy KE, Southan CD, Ryan DM, Smith TS, Simmons DL, Walsh FS, Dingwall C, Christie G (1999) Identification of a novel aspartic protease (Asp 2) as beta-secretase. Mol Cell Neurosci 14:419-427.

Iwatsubo T, Odaka A, Suzuki N, Mizusawa H, Nukina N, Ihara Y (1994) Visualization of A beta 42(43) and A beta 40 in senile plaques with end-specific A beta monoclonals: evidence that an initially deposited species is A beta 42(43). Neuron 13:45-53.

Jankowsky JL, Younkin LH, Gonzales V, Fadale DJ, Slunt HH, Lester HA, Younkin SG, Borchelt DR (2007) Rodent A beta modulates the solubility and distribution of amyloid deposits in transgenic mice. J Biol Chem 282:22707-22720.

Johansson AS, Garlind A, Berglind-Dehlin F, Karlsson G, Edwards K, Gellerfors P, EkholmPettersson F, Palmblad J, Lannfelt L (2007) Docosahexaenoic acid stabilizes soluble amyloid-beta protofibrils and sustains amyloid-beta-induced neurotoxicity in vitro. Febs J 274:990-1000.

Joyashiki E, Matsuya Y, Tohda C (2011) Sominone improves memory impairments and increases axonal density in Alzheimer's disease model mice, 5XFAD. Int J Neurosci 121:181-190.

Kalinin S, Richardson JC, Feinstein DL (2009) A PPARdelta agonist reduces amyloid burden and brain inflammation in a transgenic mouse model of Alzheimer's disease. Curr Alzheimer Res 6:431-437.

Kang J, Lemaire HG, Unterbeck A, Salbaum JM, Masters CL, Grzeschik KH, Multhaup G, Beyreuther K, Muller-Hill B (1987) The precursor of Alzheimer's disease amyloid A4 protein resembles a cell-surface receptor. Nature 325:733-736.

Kawarabayashi T, Younkin LH, Saido TC, Shoji M, Ashe KH, Younkin SG (2001) Agedependent changes in brain, CSF, and plasma amyloid (beta) protein in the Tg2576 transgenic mouse model of Alzheimer's disease. J Neurosci 21:372-381. 
Kidd M (1963) Paired helical filaments in electron microscopy of Alzheimer's disease. Nature 197:192-193.

Killiany RJ, Moss MB, Albert MS, Sandor T, Tieman J, Jolesz F (1993) Temporal lobe regions on magnetic resonance imaging identify patients with early Alzheimer's disease. Arch Neurol 50:949-954.

Kimberly WT, LaVoie MJ, Ostaszewski BL, Ye W, Wolfe MS, Selkoe DJ (2003) Gammasecretase is a membrane protein complex comprised of presenilin, nicastrin, Aph-1, and Pen-2. Proc Natl Acad Sci U S A 100:6382-6387.

Kimura R, Ohno M (2009) Impairments in remote memory stabilization precede hippocampal synaptic and cognitive failures in 5XFAD Alzheimer mouse model. Neurobiol Dis 33:229-235.

Kitaguchi N, Takahashi Y, Tokushima Y, Shiojiri S, Ito H (1988) Novel precursor of Alzheimer's disease amyloid protein shows protease inhibitory activity. Nature 331:530-532.

Klein WL, Krafft GA, Finch CE (2001) Targeting small Abeta oligomers: the solution to an Alzheimer's disease conundrum? Trends Neurosci 24:219-224.

Koike H, Tomioka S, Sorimachi H, Saido TC, Maruyama K, Okuyama A, Fujisawa-Sehara A, Ohno S, Suzuki K, Ishiura S (1999) Membrane-anchored metalloprotease MDC9 has an alpha-secretase activity responsible for processing the amyloid precursor protein. Biochem J 343 Pt 2:371-375.

Koo EH, Squazzo SL (1994) Evidence that production and release of amyloid beta-protein involves the endocytic pathway. J Biol Chem 269:17386-17389.

Koo EH, Sisodia SS, Archer DR, Martin LJ, Weidemann A, Beyreuther K, Fischer P, Masters CL, Price DL (1990) Precursor of amyloid protein in Alzheimer disease undergoes fast anterograde axonal transport. Proc Natl Acad Sci U S A 87:1561-1565.

Kowall NW, Kosik KS (1987) Axonal disruption and aberrant localization of tau protein characterize the neuropil pathology of Alzheimer's disease. Ann Neurol 22:639-643.

Kumar-Singh S, Theuns J, Van Broeck B, Pirici D, Vennekens K, Corsmit E, Cruts M, Dermaut B, Wang R, Van Broeckhoven C (2006) Mean age-of-onset of familial alzheimer disease caused by presenilin mutations correlates with both increased Abeta42 and decreased Abeta40. Hum Mutat 27:686-695.

Kuo YM, Emmerling MR, Woods AS, Cotter RJ, Roher AE (1997) Isolation, chemical characterization, and quantitation of A beta 3-pyroglutamyl peptide from neuritic plaques and vascular amyloid deposits. Biochem Biophys Res Commun 237:188-191.

Kuo YM, Kokjohn TA, Beach TG, Sue LI, Brune D, Lopez JC, Kalback WM, Abramowski D, Sturchler-Pierrat C, Staufenbiel M, Roher AE (2001) Comparative analysis of amyloid-beta chemical structure and amyloid plaque morphology of transgenic mouse and Alzheimer's disease brains. J Biol Chem 276:12991-12998.

Lalonde R, Strazielle C (2007) Brain regions and genes affecting postural control. Prog Neurobiol 81:45-60.

Lalonde R, Qian S, Strazielle C (2003a) Transgenic mice expressing the PS1-A246E mutation: effects on spatial learning, exploration, anxiety, and motor coordination. Behav Brain Res 138:71-79.

Lalonde R, Kim HD, Fukuchi K (2004) Exploratory activity, anxiety, and motor coordination in bigenic APPswe + PS1/DeltaE9 mice. Neurosci Lett 369:156-161. 
Lalonde R, Dumont M, Staufenbiel M, Strazielle C (2005) Neurobehavioral characterization of APP23 transgenic mice with the SHIRPA primary screen. Behav Brain Res 157:9198.

Lalonde R, Dumont M, Staufenbiel M, Sturchler-Pierrat C, Strazielle C (2002) Spatial learning, exploration, anxiety, and motor coordination in female APP23 transgenic mice with the Swedish mutation. Brain Res 956:36-44.

Lalonde R, Lewis TL, Strazielle C, Kim H, Fukuchi K (2003b) Transgenic mice expressing the betaAPP695SWE mutation: effects on exploratory activity, anxiety, and motor coordination. Brain Res 977:38-45.

Lammich S, Kojro E, Postina R, Gilbert S, Pfeiffer R, Jasionowski M, Haass C, Fahrenholz F (1999) Constitutive and regulated alpha-secretase cleavage of Alzheimer's amyloid precursor protein by a disintegrin metalloprotease. Proc Natl Acad Sci U S A 96:39223927.

Lange-Asschenfeldt C, Kojda G (2008) Alzheimer's disease, cerebrovascular dysfunction and the benefits of exercise: from vessels to neurons. Exp Gerontol 43:499-504.

Lassalle JM, Halley H, Daumas S, Verret L, Frances B (2008) Effects of the genetic background on cognitive performances of TG2576 mice. Behav Brain Res 191:104110.

Le Cudennec C, Faure A, Ly M, Delatour B (2008) One-year longitudinal evaluation of sensorimotor functions in APP751SL transgenic mice. Genes Brain Behav 7 Suppl $1: 83-91$.

Lee KW, Lee SH, Kim H, Song JS, Yang SD, Paik SG, Han PL (2004) Progressive cognitive impairment and anxiety induction in the absence of plaque deposition in C57BL/6 inbred mice expressing transgenic amyloid precursor protein. J Neurosci Res 76:572580.

Lee MS, Kao SC, Lemere CA, Xia W, Tseng HC, Zhou Y, Neve R, Ahlijanian MK, Tsai LH (2003) APP processing is regulated by cytoplasmic phosphorylation. J Cell Biol 163:83-95.

Lee VM, Balin BJ, Otvos L, Jr., Trojanowski JQ (1991) A68: a major subunit of paired helical filaments and derivatized forms of normal Tau. Science 251:675-678.

Lehman EJ, Kulnane LS, Gao Y, Petriello MC, Pimpis KM, Younkin L, Dolios G, Wang R, Younkin SG, Lamb BT (2003) Genetic background regulates beta-amyloid precursor protein processing and beta-amyloid deposition in the mouse. Hum Mol Genet 12:2949-2956.

Lemere CA, Blusztajn JK, Yamaguchi H, Wisniewski T, Saido TC, Selkoe DJ (1996) Sequence of deposition of heterogeneous amyloid beta-peptides and APO E in Down syndrome: implications for initial events in amyloid plaque formation. Neurobiol Dis 3:16-32.

Lemmens MA, Sierksma AS, Rutten BP, Dennissen F, Steinbusch HW, Lucassen PJ, Schmitz $C$ (2011) Age-related changes of neuron numbers in the frontal cortex of a transgenic mouse model of Alzheimer's disease. Brain Struct Funct. Advance online publication. Retrieved August 21, 2011. doi: 10.1007/s00429-011-0305-1.

Lewis J, Dickson DW, Lin WL, Chisholm L, Corral A, Jones G, Yen SH, Sahara N, Skipper L, Yager D, Eckman C, Hardy J, Hutton M, McGowan E (2001) Enhanced 
neurofibrillary degeneration in transgenic mice expressing mutant tau and APP. Science 293:1487-1491.

Lindsay J, Laurin D, Verreault R, Hebert R, Helliwell B, Hill GB, McDowell I (2002) Risk factors for Alzheimer's disease: a prospective analysis from the Canadian Study of Health and Aging. Am J Epidemiol 156:445-453.

Linn RT, Wolf PA, Bachman DL, Knoefel JE, Cobb JL, Belanger AJ, Kaplan EF, D'Agostino RB (1995) The 'preclinical phase' of probable Alzheimer's disease. A 13-year prospective study of the Framingham cohort. Arch Neurol 52:485-490.

Locascio JJ, Growdon JH, Corkin S (1995) Cognitive test performance in detecting, staging, and tracking Alzheimer's disease. Arch Neurol 52:1087-1099.

Manczak M, Anekonda TS, Henson E, Park BS, Quinn J, Reddy PH (2006) Mitochondria are a direct site of $\mathrm{A}$ beta accumulation in Alzheimer's disease neurons: implications for free radical generation and oxidative damage in disease progression. Hum Mol Genet 15:1437-1449.

Mandelkow EM, Drewes G, Biernat J, Gustke N, Van Lint J, Vandenheede JR, Mandelkow E (1992) Glycogen synthase kinase-3 and the Alzheimer-like state of microtubuleassociated protein tau. FEBS Lett 314:315-321.

Masters CL, Multhaup G, Simms G, Pottgiesser J, Martins RN, Beyreuther K (1985a) Neuronal origin of a cerebral amyloid: neurofibrillary tangles of Alzheimer's disease contain the same protein as the amyloid of plaque cores and blood vessels. Embo J 4:2757-2763.

Masters CL, Simms G, Weinman NA, Multhaup G, McDonald BL, Beyreuther K (1985b) Amyloid plaque core protein in Alzheimer disease and Down syndrome. Proc Natl Acad Sci U S A 82:4245-4249.

Mattsson N, Blennow K, Zetterberg H (2009) CSF biomarkers: pinpointing Alzheimer pathogenesis. Ann N Y Acad Sci 1180:28-35.

Mayeux R (2003) Epidemiology of neurodegeneration. Annu Rev Neurosci 26:81-104.

McNeill TH, Koek LL, Haycock JW (1984) The nigrostriatal system and aging. Peptides 5 Suppl 1:263-268.

Mega MS, Cummings JL, Fiorello T, Gornbein J (1996) The spectrum of behavioral changes in Alzheimer's disease. Neurology 46:130-135.

Messer M (1963) Enzymatic cyclization of L-glutamine and L-glutaminyl peptides. Nature 197:1299.

Miller DL, Papayannopoulos IA, Styles J, Bobin SA, Lin YY, Biemann K, Iqbal K (1993) Peptide compositions of the cerebrovascular and senile plaque core amyloid deposits of Alzheimer's disease. Arch Biochem Biophys 301:41-52.

Mohamed A, Posse de Chaves E (2011) Abeta internalization by neurons and glia. Int J Alzheimers Dis 2011:127984.

Moore V, Wyke MA (1984) Drawing disability in patients with senile dementia. Psychol Med 14:97-105.

Moran PM, Higgins LS, Cordell B, Moser PC (1995) Age-related learning deficits in transgenic mice expressing the 751-amino acid isoform of human beta-amyloid precursor protein. Proc Natl Acad Sci U S A 92:5341-5345.

Mori H, Takio K, Ogawara M, Selkoe DJ (1992) Mass spectrometry of purified amyloid beta protein in Alzheimer's disease. J Biol Chem 267:17082-17086. 
Morrison JH, Hof PR (2007) Life and death of neurons in the aging cerebral cortex. Int Rev Neurobiol 81:41-57.

Morty RE, Bulau P, Pelle R, Wilk S, Abe K (2006) Pyroglutamyl peptidase type I from Trypanosoma brucei: a new virulence factor from African trypanosomes that de-blocks regulatory peptides in the plasma of infected hosts. Biochem J 394:635-645.

Mullan M (1991) A genetic defect causing Alzheimer's disease. Br J Hosp Med 45:131.

Munoz DG, Wang D (1992) Tangle-associated neuritic clusters. A new lesion in Alzheimer's disease and aging suggests that aggregates of dystrophic neurites are not necessarily associated with beta/A4. Am J Pathol 140:1167-1178.

Naslund J, Karlstrom AR, Tjernberg LO, Schierhorn A, Terenius L, Nordstedt C (1996) Highresolution separation of amyloid beta-peptides: structural variants present in Alzheimer's disease amyloid. J Neurochem 67:294-301.

Naslund J, Schierhorn A, Hellman U, Lannfelt L, Roses AD, Tjernberg LO, Silberring J, Gandy SE, Winblad B, Greengard P, et al. (1994) Relative abundance of Alzheimer A beta amyloid peptide variants in Alzheimer disease and normal aging. Proc Natl Acad Sci U S A 91:8378-8382.

Neve RL, Harris P, Kosik KS, Kurnit DM, Donlon TA (1986) Identification of cDNA clones for the human microtubule-associated protein tau and chromosomal localization of the genes for tau and microtubule-associated protein 2. Brain Res 387:271-280.

Nilsberth C, Westlind-Danielsson A, Eckman CB, Condron MM, Axelman K, Forsell C, Stenh C, Luthman J, Teplow DB, Younkin SG, Naslund J, Lannfelt L (2001) The 'Arctic' APP mutation (E693G) causes Alzheimer's disease by enhanced Abeta protofibril formation. Nat Neurosci 4:887-893.

Norfray JF, Provenzale JM (2004) Alzheimer's disease: neuropathologic findings and recent advances in imaging. AJR Am J Roentgenol 182:3-13.

O'Keeffe ST, Kazeem H, Philpott RM, Playfer JR, Gosney M, Lye M (1996) Gait disturbance in Alzheimer's disease: a clinical study. Age Ageing 25:313-316.

Oakley H, Cole SL, Logan S, Maus E, Shao P, Craft J, Guillozet-Bongaarts A, Ohno M, Disterhoft J, Van Eldik L, Berry R, Vassar R (2006) Intraneuronal beta-amyloid aggregates, neurodegeneration, and neuron loss in transgenic mice with five familial Alzheimer's disease mutations: potential factors in amyloid plaque formation. J Neurosci 26:10129-10140.

Oddo S, Caccamo A, Shepherd JD, Murphy MP, Golde TE, Kayed R, Metherate R, Mattson MP, Akbari Y, LaFerla FM (2003) Triple-transgenic model of Alzheimer's disease with plaques and tangles: intracellular Abeta and synaptic dysfunction. Neuron 39:409-421.

Ognibene E, Middei S, Daniele S, Adriani W, Ghirardi O, Caprioli A, Laviola G (2005) Aspects of spatial memory and behavioral disinhibition in $\mathrm{Tg} 2576$ transgenic mice as a model of Alzheimer's disease. Behav Brain Res 156:225-232.

Ogomori K, Kitamoto T, Tateishi J, Sato Y, Suetsugu M, Abe M (1989) Beta-protein amyloid is widely distributed in the central nervous system of patients with Alzheimer's disease. Am J Pathol 134:243-251.

Ohno M, Cole SL, Yasvoina M, Zhao J, Citron M, Berry R, Disterhoft JF, Vassar R (2007) BACE1 gene deletion prevents neuron loss and memory deficits in 5XFAD APP/PS1 transgenic mice. Neurobiol Dis 26:134-145. 
Petersen RC (2004) Challenges of epidemiological studies of mild cognitive impairment. Alzheimer Dis Assoc Disord 18:1-2.

Philipson O, Lord A, Gumucio A, O'Callaghan P, Lannfelt L, Nilsson LN (2010) Animal models of amyloid-beta-related pathologies in Alzheimer's disease. Febs J 277:13891409.

Piccini A, Russo C, Gliozzi A, Relini A, Vitali A, Borghi R, Giliberto L, Armirotti A, D'Arrigo C, Bachi A, Cattaneo A, Canale C, Torrassa S, Saido TC, Markesbery W, Gambetti P, Tabaton M (2005) beta-amyloid is different in normal aging and in Alzheimer disease. J Biol Chem 280:34186-34192.

Pietropaolo S, Feldon J, Yee BK (2008) Age-dependent phenotypic characteristics of a triple transgenic mouse model of Alzheimer disease. Behav Neurosci 122:733-747.

Pike CJ, Overman MJ, Cotman CW (1995) Amino-terminal deletions enhance aggregation of beta-amyloid peptides in vitro. J Biol Chem 270:23895-23898.

Pohl T, Zimmer M, Mugele K, Spiess J (1991) Primary structure and functional expression of a glutaminyl cyclase. Proc Natl Acad Sci U S A 88:10059-10063.

Prelli F, Castano E, Glenner GG, Frangione B (1988) Differences between vascular and plaque core amyloid in Alzheimer's disease. J Neurochem 51:648-651.

Probst A, Basler V, Bron B, Ulrich J (1983) Neuritic plaques in senile dementia of Alzheimer type: a Golgi analysis in the hippocampal region. Brain Res 268:249-254.

Probst A, Gotz J, Wiederhold KH, Tolnay M, Mistl C, Jaton AL, Hong M, Ishihara T, Lee VM, Trojanowski JQ, Jakes R, Crowther RA, Spillantini MG, Burki K, Goedert M (2000) Axonopathy and amyotrophy in mice transgenic for human four-repeat tau protein. Acta Neuropathol 99:469-481.

Pugh PL, Richardson JC, Bate ST, Upton N, Sunter D (2007) Non-cognitive behaviours in an APP/PS1 transgenic model of Alzheimer's disease. Behav Brain Res 178:18-28.

Rebeck GW, Hoe HS, Moussa CE (2010) Beta-amyloid1-42 gene transfer model exhibits intraneuronal amyloid, gliosis, tau phosphorylation, and neuronal loss. J Biol Chem 285:7440-7446.

Roberts SB, Ripellino JA, Ingalls KM, Robakis NK, Felsenstein KM (1994) Nonamyloidogenic cleavage of the beta-amyloid precursor protein by an integral membrane metalloendopeptidase. J Biol Chem 269:3111-3116.

Rockenstein E, Mallory M, Mante M, Sisk A, Masliaha E (2001) Early formation of mature amyloid-beta protein deposits in a mutant APP transgenic model depends on levels of Abeta(1-42). J Neurosci Res 66:573-582.

Rockenstein EM, McConlogue L, Tan H, Power M, Masliah E, Mucke L (1995) Levels and alternative splicing of amyloid beta protein precursor (APP) transcripts in brains of APP transgenic mice and humans with Alzheimer's disease. J Biol Chem 270:2825728267.

Roher AE, Lowenson JD, Clarke S, Woods AS, Cotter RJ, Gowing E, Ball MJ (1993) betaAmyloid-(1-42) is a major component of cerebrovascular amyloid deposits: implications for the pathology of Alzheimer disease. Proc Natl Acad Sci U S A 90:10836-10840.

Romanelli MF, Morris JC, Ashkin K, Coben LA (1990) Advanced Alzheimer's disease is a risk factor for late-onset seizures. Arch Neurol 47:847-850. 
Roychaudhuri R, Yang M, Hoshi MM, Teplow DB (2009) Amyloid beta-protein assembly and Alzheimer disease. J Biol Chem 284:4749-4753.

Russo C, Saido TC, DeBusk LM, Tabaton M, Gambetti P, Teller JK (1997) Heterogeneity of water-soluble amyloid beta-peptide in Alzheimer's disease and Down's syndrome brains. FEBS Lett 409:411-416.

Russo C, Salis S, Dolcini V, Venezia V, Song XH, Teller JK, Schettini G (2001) Aminoterminal modification and tyrosine phosphorylation of [corrected] carboxy-terminal fragments of the amyloid precursor protein in Alzheimer's disease and Down's syndrome brain. Neurobiol Dis 8:173-180.

Russo C, Violani E, Salis S, Venezia V, Dolcini V, Damonte G, Benatti U, D'Arrigo C, Patrone E, Carlo P, Schettini G (2002) Pyroglutamate-modified amyloid beta-peptides-AbetaN3(pE)--strongly affect cultured neuron and astrocyte survival. J Neurochem 82:1480-1489.

Saido TC, Yamao-Harigaya W, Iwatsubo T, Kawashima S (1996) Amino- and carboxylterminal heterogeneity of beta-amyloid peptides deposited in human brain. Neurosci Lett 215:173-176.

Saido TC, Iwatsubo T, Mann DM, Shimada H, Ihara Y, Kawashima S (1995) Dominant and differential deposition of distinct beta-amyloid peptide species, A beta N3(pE), in senile plaques. Neuron 14:457-466.

Saito Y, Murayama S (2000) Expression of tau immunoreactivity in the spinal motor neurons of Alzheimer's disease. Neurology 55:1727-1729.

Salehi A, Delcroix JD, Belichenko PV, Zhan K, Wu C, Valletta JS, Takimoto-Kimura R, Kleschevnikov AM, Sambamurti K, Chung PP, Xia W, Villar A, Campbell WA, Kulnane LS, Nixon RA, Lamb BT, Epstein CJ, Stokin GB, Goldstein LS, Mobley WC (2006) Increased App expression in a mouse model of Down's syndrome disrupts NGF transport and causes cholinergic neuron degeneration. Neuron 51:29-42.

Savonenko AV, Xu GM, Price DL, Borchelt DR, Markowska AL (2003) Normal cognitive behavior in two distinct congenic lines of transgenic mice hyperexpressing mutant APP SWE. Neurobiol Dis 12:194-211.

Scarmeas N, Levy G, Tang MX, Manly J, Stern Y (2001) Influence of leisure activity on the incidence of Alzheimer's disease. Neurology 57:2236-2242.

Scarmeas N, Hadjigeorgiou GM, Papadimitriou A, Dubois B, Sarazin M, Brandt J, Albert M, Marder K, Bell K, Honig LS, Wegesin D, Stern Y (2004) Motor signs during the course of Alzheimer disease. Neurology 63:975-982.

Scheff SW, Price DA, Schmitt FA, DeKosky ST, Mufson EJ (2007) Synaptic alterations in CA1 in mild Alzheimer disease and mild cognitive impairment. Neurology 68:15011508.

Schieb H, Kratzin H, Jahn O, Moebius W, Rabe S, Staufenbiel M, Wiltfang J, Klafki HW (2011) \{beta\}-amyloid peptide variants in brains and cerebrospinal fluid from APP transgenic mice: Comparison with human Alzheimer's amyloid. J Biol Chem.

Schilling S, Wasternack C, Demuth HU (2008a) Glutaminyl cyclases from animals and plants: a case of functionally convergent protein evolution. Biol Chem 389:983-991.

Schilling S, Hoffmann T, Manhart S, Hoffmann M, Demuth HU (2004) Glutaminyl cyclases unfold glutamyl cyclase activity under mild acid conditions. FEBS Lett 563:191-196. 
Schilling S, Lauber T, Schaupp M, Manhart S, Scheel E, Bohm G, Demuth HU (2006) On the seeding and oligomerization of pGlu-amyloid peptides (in vitro). Biochemistry 45:12393-12399.

Schilling S, Stenzel I, von Bohlen A, Wermann M, Schulz K, Demuth HU, Wasternack C (2007) Isolation and characterization of the glutaminyl cyclases from Solanum tuberosum and Arabidopsis thaliana: implications for physiological functions. Biol Chem 388:145-153.

Schilling S, Niestroj AJ, Rahfeld JU, Hoffmann T, Wermann M, Zunkel K, Wasternack C, Demuth HU (2003) Identification of human glutaminyl cyclase as a metalloenzyme. Potent inhibition by imidazole derivatives and heterocyclic chelators. J Biol Chem 278:49773-49779.

Schilling S, Appl T, Hoffmann T, Cynis H, Schulz K, Jagla W, Friedrich D, Wermann M, Buchholz M, Heiser U, von Horsten S, Demuth HU (2008b) Inhibition of glutaminyl cyclase prevents pGlu-Abeta formation after intracortical/hippocampal microinjection in vivo/in situ. J Neurochem 106:1225-1236.

Schilling S, Kohlmann S, Bauscher C, Sedlmeier R, Koch B, Eichentopf R, Becker A, Cynis H, Hoffmann T, Berg S, Freyse EJ, von Horsten S, Rossner S, Graubner S, Demuth HU (2011) Glutaminyl cyclase knock-out mice exhibit slight hypothyroidism but no hypogonadism: implications for enzyme function and drug development. J Biol Chem 286:14199-14208.

Schilling S, Zeitschel U, Hoffmann T, Heiser U, Francke M, Kehlen A, Holzer M, HutterPaier B, Prokesch M, Windisch M, Jagla W, Schlenzig D, Lindner C, Rudolph T, Reuter G, Cynis H, Montag D, Demuth HU, Rossner S (2008c) Glutaminyl cyclase inhibition attenuates pyroglutamate Abeta and Alzheimer's disease-like pathology. Nat Med 14:1106-1111.

Schmitz C, Rutten BP, Pielen A, Schafer S, Wirths O, Tremp G, Czech C, Blanchard V, Multhaup G, Rezaie P, Korr H, Steinbusch HW, Pradier L, Bayer TA (2004) Hippocampal neuron loss exceeds amyloid plaque load in a transgenic mouse model of Alzheimer's disease. Am J Pathol 164:1495-1502.

Sealfon SC, Weinstein H, Millar RP (1997) Molecular mechanisms of ligand interaction with the gonadotropin-releasing hormone receptor. Endocr Rev 18:180-205.

Seifert F, Schulz K, Koch B, Manhart S, Demuth HU, Schilling S (2009) Glutaminyl cyclases display significant catalytic proficiency for glutamyl substrates. Biochemistry 48:11831-11833.

Selkoe DJ (2001) Alzheimer's disease: genes, proteins, and therapy. Physiol Rev 81:741-766.

Selkoe DJ (2008) Soluble oligomers of the amyloid beta-protein impair synaptic plasticity and behavior. Behav Brain Res 192:106-113.

Selkoe DJ, Abraham CR, Podlisny MB, Duffy LK (1986) Isolation of low-molecular-weight proteins from amyloid plaque fibers in Alzheimer's disease. J Neurochem 46:18201834.

Seo JS, Leem YH, Lee KW, Kim SW, Lee JK, Han PL (2010) Severe motor neuron degeneration in the spinal cord of the Tg2576 mouse model of Alzheimer disease. $\mathrm{J}$ Alzheimers Dis 21:263-276. 
Sevalle J, Amoyel A, Robert P, Fournie-Zaluski MC, Roques B, Checler F (2009) Aminopeptidase A contributes to the N-terminal truncation of amyloid beta-peptide. $\mathrm{J}$ Neurochem 109:248-256.

Shao CY, Mirra SS, Sait HB, Sacktor TC, Sigurdsson EM (2011) Postsynaptic degeneration as revealed by PSD-95 reduction occurs after advanced Abeta and tau pathology in transgenic mouse models of Alzheimer's disease. Acta Neuropathol.122:285-292.

Shin RW, Ogino K, Kondo A, Saido TC, Trojanowski JQ, Kitamoto T, Tateishi J (1997) Amyloid beta-protein (Abeta) 1-40 but not Abeta1-42 contributes to the experimental formation of Alzheimer disease amyloid fibrils in rat brain. J Neurosci 17:8187-8193.

Silverman DH, Small GW, Chang CY, Lu CS, Kung De Aburto MA, Chen W, Czernin J, Rapoport SI, Pietrini P, Alexander GE, Schapiro MB, Jagust WJ, Hoffman JM, WelshBohmer KA, Alavi A, Clark CM, Salmon E, de Leon MJ, Mielke R, Cummings JL, Kowell AP, Gambhir SS, Hoh CK, Phelps ME (2001) Positron emission tomography in evaluation of dementia: Regional brain metabolism and long-term outcome. Jama 286:2120-2127.

Sinha S, Anderson JP, Barbour R, Basi GS, Caccavello R, Davis D, Doan M, Dovey HF, Frigon N, Hong J, Jacobson-Croak K, Jewett N, Keim P, Knops J, Lieberburg I, Power M, Tan H, Tatsuno G, Tung J, Schenk D, Seubert P, Suomensaari SM, Wang S, Walker D, Zhao J, McConlogue L, John V (1999) Purification and cloning of amyloid precursor protein beta-secretase from human brain. Nature 402:537-540.

Sisodia SS (1992) Beta-amyloid precursor protein cleavage by a membrane-bound protease. Proc Natl Acad Sci U S A 89:6075-6079.

Sisodia SS, Koo EH, Beyreuther K, Unterbeck A, Price DL (1990) Evidence that betaamyloid protein in Alzheimer's disease is not derived by normal processing. Science 248:492-495.

Sleegers K, Lambert JC, Bertram L, Cruts M, Amouyel P, Van Broeckhoven C (2010) The pursuit of susceptibility genes for Alzheimer's disease: progress and prospects. Trends Genet 26:84-93.

Small GW (1998) The pathogenesis of Alzheimer's disease. J Clin Psychiatry 59 Suppl 9:714.

Smith KD, Kallhoff V, Zheng H, Pautler RG (2007) In vivo axonal transport rates decrease in a mouse model of Alzheimer's disease. Neuroimage 35:1401-1408.

Snowdon DA (2003) Healthy aging and dementia: findings from the Nun Study. Ann Intern Med 139:450-454.

Sola C, Mengod G, Probst A, Palacios JM (1993) Differential regional and cellular distribution of beta-amyloid precursor protein messenger RNAs containing and lacking the Kunitz protease inhibitor domain in the brain of human, rat and mouse. Neuroscience 53:267-295.

Song I, Chuang CZ, Bateman RC, Jr. (1994) Molecular cloning, sequence analysis and expression of human pituitary glutaminyl cyclase. J Mol Endocrinol 13:77-86.

Spittaels K, Van den Haute C, Van Dorpe J, Bruynseels K, Vandezande K, Laenen I, Geerts H, Mercken M, Sciot R, Van Lommel A, Loos R, Van Leuven F (1999) Prominent axonopathy in the brain and spinal cord of transgenic mice overexpressing four-repeat human tau protein. Am J Pathol 155:2153-2165. 
St George-Hyslop PH, Tanzi RE, Polinsky RJ, Haines JL, Nee L, Watkins PC, Myers RH, Feldman RG, Pollen D, Drachman D, et al. (1987) The genetic defect causing familial Alzheimer's disease maps on chromosome 21. Science 235:885-890.

Stephan A, Wermann M, von Bohlen A, Koch B, Cynis H, Demuth HU, Schilling S (2009) Mammalian glutaminyl cyclases and their isoenzymes have identical enzymatic characteristics. Febs J 276:6522-6536.

Stokin GB, Lillo C, Falzone TL, Brusch RG, Rockenstein E, Mount SL, Raman R, Davies P, Masliah E, Williams DS, Goldstein LS (2005) Axonopathy and transport deficits early in the pathogenesis of Alzheimer's disease. Science 307:1282-1288.

$\mathrm{Su}$ JH, Cummings BJ, Cotman CW (1998) Plaque biogenesis in brain aging and Alzheimer's disease. II. Progressive transformation and developmental sequence of dystrophic neurites. Acta Neuropathol 96:463-471.

Suzuki N, Iwatsubo T, Odaka A, Ishibashi Y, Kitada C, Ihara Y (1994a) High tissue content of soluble beta 1-40 is linked to cerebral amyloid angiopathy. Am J Pathol 145:452460.

Suzuki N, Cheung TT, Cai XD, Odaka A, Otvos L, Jr., Eckman C, Golde TE, Younkin SG (1994b) An increased percentage of long amyloid beta protein secreted by familial amyloid beta protein precursor (beta APP717) mutants. Science 264:1336-1340.

Suzuki T, Nakaya T (2008) Regulation of amyloid beta-protein precursor by phosphorylation and protein interactions. J Biol Chem 283:29633-29637.

Suzuki T, Ando K, Isohara T, Oishi M, Lim GS, Satoh Y, Wasco W, Tanzi RE, Nairn AC, Greengard P, Gandy SE, Kirino Y (1997) Phosphorylation of Alzheimer beta-amyloid precursor-like proteins. Biochemistry 36:4643-4649.

Takahashi RH, Milner TA, Li F, Nam EE, Edgar MA, Yamaguchi H, Beal MF, Xu H, Greengard P, Gouras GK (2002) Intraneuronal Alzheimer abeta42 accumulates in multivesicular bodies and is associated with synaptic pathology. Am J Pathol 161:1869-1879.

Tamura BK, Masaki KH, Blanchette P (2007) Weight loss in patients with Alzheimer's disease. J Nutr Elder 26:21-38.

Tang BL (2009) Neuronal protein trafficking associated with Alzheimer disease: from APP and BACE1 to glutamate receptors. Cell Adh Migr 3:118-128.

Tanghe A, Termont A, Merchiers P, Schilling S, Demuth HU, Scrocchi L, Van Leuven F, Griffioen G, Van Dooren T (2010) Pathological Hallmarks, Clinical Parallels, and Value for Drug Testing in Alzheimer's Disease of the APP[V717I] London Transgenic Mouse Model. Int J Alzheimers Dis. Advance online publication. Retrieved August 21, 2011. doi: 10.4061/2010/417314.

Tanzi RE, McClatchey AI, Lamperti ED, Villa-Komaroff L, Gusella JF, Neve RL (1988) Protease inhibitor domain encoded by an amyloid protein precursor mRNA associated with Alzheimer's disease. Nature 331:528-530.

Tanzi RE, Gusella JF, Watkins PC, Bruns GA, St George-Hyslop P, Van Keuren ML, Patterson D, Pagan S, Kurnit DM, Neve RL (1987) Amyloid beta protein gene: cDNA, mRNA distribution, and genetic linkage near the Alzheimer locus. Science 235:880884. 
Tekirian TL, Yang AY, Glabe C, Geddes JW (1999) Toxicity of pyroglutaminated amyloid beta-peptides $3(\mathrm{pE})-40$ and -42 is similar to that of A beta1-40 and -42 . J Neurochem 73:1584-1589.

Tekirian TL, Saido TC, Markesbery WR, Russell MJ, Wekstein DR, Patel E, Geddes JW (1998) N-terminal heterogeneity of parenchymal and cerebrovascular Abeta deposits. J Neuropathol Exp Neurol 57:76-94.

ter Laak HJ, Renkawek K, van Workum FP (1994) The olfactory bulb in Alzheimer disease: a morphologic study of neuron loss, tangles, and senile plaques in relation to olfaction. Alzheimer Dis Assoc Disord 8:38-48.

Terry AV, Jr., Buccafusco JJ (2003) The cholinergic hypothesis of age and Alzheimer's disease-related cognitive deficits: recent challenges and their implications for novel drug development. J Pharmacol Exp Ther 306:821-827.

Terry RD, Gonatas NK, Weiss M (1964) Ultrastructural Studies in Alzheimer's Presenile Dementia. Am J Pathol 44:269-297.

Terry RD, Masliah E, Salmon DP, Butters N, DeTeresa R, Hill R, Hansen LA, Katzman R (1991) Physical basis of cognitive alterations in Alzheimer's disease: synapse loss is the major correlate of cognitive impairment. Ann Neurol 30:572-580.

Tesseur I, Van Dorpe J, Bruynseels K, Bronfman F, Sciot R, Van Lommel A, Van Leuven F (2000) Prominent axonopathy and disruption of axonal transport in transgenic mice expressing human apolipoprotein E4 in neurons of brain and spinal cord. Am J Pathol 157:1495-1510.

Thompson AJ, Lim TK, Barrow CJ (1999) On-line high-performance liquid chromatography/mass spectrometric investigation of amyloid-beta peptide variants found in Alzheimer's disease. Rapid Commun Mass Spectrom 13:2348-2351.

Thompson PM, Mega MS, Woods RP, Zoumalan CI, Lindshield CJ, Blanton RE, Moussai J, Holmes CJ, Cummings JL, Toga AW (2001) Cortical change in Alzheimer's disease detected with a disease-specific population-based brain atlas. Cereb Cortex 11:1-16.

Tomita S, Kirino Y, Suzuki T (1998) Cleavage of Alzheimer's amyloid precursor protein (APP) by secretases occurs after O-glycosylation of APP in the protein secretory pathway. Identification of intracellular compartments in which APP cleavage occurs without using toxic agents that interfere with protein metabolism. J Biol Chem 273:6277-6284.

Tomiyama T, Matsuyama S, Iso H, Umeda T, Takuma H, Ohnishi K, Ishibashi K, Teraoka R, Sakama N, Yamashita T, Nishitsuji K, Ito K, Shimada H, Lambert MP, Klein WL, Mori H (2010) A mouse model of amyloid beta oligomers: their contribution to synaptic alteration, abnormal tau phosphorylation, glial activation, and neuronal loss in vivo. J Neurosci 30:4845-4856.

Trobe JD, Waller PF, Cook-Flannagan CA, Teshima SM, Bieliauskas LA (1996) Crashes and violations among drivers with Alzheimer disease. Arch Neurol 53:411-416.

Tseng BP, Green KN, Chan JL, Blurton-Jones M, LaFerla FM (2008) Abeta inhibits the proteasome and enhances amyloid and tau accumulation. Neurobiol Aging 29:16071618.

Turner RS, Suzuki N, Chyung AS, Younkin SG, Lee VM (1996) Amyloids beta40 and beta42 are generated intracellularly in cultured human neurons and their secretion increases with maturation. J Biol Chem 271:8966-8970. 
Uchihara T, Kondo H, Kosaka K, Tsukagoshi H (1992) Selective loss of nigral neurons in Alzheimer's disease: a morphometric study. Acta Neuropathol 83:271-276.

Urano T, Tohda C (2010) Icariin improves memory impairment in Alzheimer's disease model mice $(5 x F A D)$ and attenuates amyloid beta-induced neurite atrophy. Phytother Res 24:1658-1663.

Urbanc B, Cruz L, Le R, Sanders J, Ashe KH, Duff K, Stanley HE, Irizarry MC, Hyman BT (2002) Neurotoxic effects of thioflavin S-positive amyloid deposits in transgenic mice and Alzheimer's disease. Proc Natl Acad Sci U S A 99:13990-13995.

Van Coillie E, Proost P, Van Aelst I, Struyf S, Polfliet M, De Meester I, Harvey DJ, Van Damme J, Opdenakker G (1998) Functional comparison of two human monocyte chemotactic protein-2 isoforms, role of the amino-terminal pyroglutamic acid and processing by CD26/dipeptidyl peptidase IV. Biochemistry 37:12672-12680.

Van Dam D, D'Hooge R, Staufenbiel M, Van Ginneken C, Van Meir F, De Deyn PP (2003) Age-dependent cognitive decline in the APP23 model precedes amyloid deposition. Eur J Neurosci 17:388-396.

Vassar R, Bennett BD, Babu-Khan S, Kahn S, Mendiaz EA, Denis P, Teplow DB, Ross S, Amarante P, Loeloff R, Luo Y, Fisher S, Fuller J, Edenson S, Lile J, Jarosinski MA, Biere AL, Curran E, Burgess T, Louis JC, Collins F, Treanor J, Rogers G, Citron M (1999) Beta-secretase cleavage of Alzheimer's amyloid precursor protein by the transmembrane aspartic protease BACE. Science 286:735-741.

Vereecken TH, Vogels OJ, Nieuwenhuys R (1994) Neuron loss and shrinkage in the amygdala in Alzheimer's disease. Neurobiol Aging 15:45-54.

Waldemar G, Dubois B, Emre M, Georges J, McKeith IG, Rossor M, Scheltens P, Tariska P, Winblad B (2007) Recommendations for the diagnosis and management of Alzheimer's disease and other disorders associated with dementia: EFNS guideline. Eur J Neurol 14:e1-26.

Walker JM, Fowler SW, Miller DK, Sun AY, Weisman GA, Wood WG, Sun GY, Simonyi A, Schachtman TR (2011) Spatial learning and memory impairment and increased locomotion in a transgenic amyloid precursor protein mouse model of Alzheimer's disease. Behav Brain Res 222:169-175.

Walsh DM, Selkoe DJ (2007) A beta oligomers - a decade of discovery. J Neurochem 101:1172-1184.

Wertkin AM, Turner RS, Pleasure SJ, Golde TE, Younkin SG, Trojanowski JQ, Lee VM (1993) Human neurons derived from a teratocarcinoma cell line express solely the 695-amino acid amyloid precursor protein and produce intracellular beta-amyloid or A4 peptides. Proc Natl Acad Sci U S A 90:9513-9517.

West MJ, Kawas CH, Stewart WF, Rudow GL, Troncoso JC (2004) Hippocampal neurons in pre-clinical Alzheimer's disease. Neurobiol Aging 25:1205-1212.

Whalley LJ, Starr JM, Athawes R, Hunter D, Pattie A, Deary IJ (2000) Childhood mental ability and dementia. Neurology 55:1455-1459.

Winblad B, Kilander L, Eriksson S, Minthon L, Batsman S, Wetterholm AL, Jansson-Blixt C, Haglund A (2006) Donepezil in patients with severe Alzheimer's disease: doubleblind, parallel-group, placebo-controlled study. Lancet 367:1057-1065. 
Wintjens R, Belrhali H, Clantin B, Azarkan M, Bompard C, Baeyens-Volant D, Looze Y, Villeret V (2006) Crystal structure of papaya glutaminyl cyclase, an archetype for plant and bacterial glutaminyl cyclases. J Mol Biol 357:457-470.

Wirths O, Bayer TA (2008) Motor impairment in Alzheimer's disease and transgenic Alzheimer's disease mouse models. Genes Brain Behav 7 Suppl 1:1-5.

Wirths O, Bayer TA (2010) Neuron loss in transgenic mouse models of Alzheimer's disease. Int J Alzheimers Dis 2010. Advance online publication. Retrieved August 21, 2011. doi: $10.4061 / 2010 / 723782$.

Wirths O, Multhaup G, Bayer TA (2004) A modified beta-amyloid hypothesis: intraneuronal accumulation of the beta-amyloid peptide--the first step of a fatal cascade. $\mathrm{J}$ Neurochem 91:513-520.

Wirths O, Weis J, Szczygielski J, Multhaup G, Bayer TA (2006) Axonopathy in an APP/PS1 transgenic mouse model of Alzheimer's disease. Acta Neuropathol 111:312-319.

Wirths O, Weis J, Kayed R, Saido TC, Bayer TA (2007) Age-dependent axonal degeneration in an Alzheimer mouse model. Neurobiol Aging 28:1689-1699.

Wirths O, Breyhan H, Cynis H, Schilling S, Demuth HU, Bayer TA (2009) Intraneuronal pyroglutamate-Abeta 3-42 triggers neurodegeneration and lethal neurological deficits in a transgenic mouse model. Acta Neuropathol 118:487-496.

Wirths O, Bethge T, Marcello A, Harmeier A, Jawhar S, Lucassen PJ, Multhaup G, Brody DL, Esparza T, Ingelsson M, Kalimo H, Lannfelt L, Bayer TA (2010a) Pyroglutamate Abeta pathology in APP/PS1KI mice, sporadic and familial Alzheimer's disease cases. J Neural Transm 117:85-96.

Wirths O, Erck C, Martens H, Harmeier A, Geumann C, Jawhar S, Kumar S, Multhaup G, Walter J, Ingelsson M, Degerman-Gunnarsson M, Kalimo H, Huitinga I, Lannfelt L, Bayer TA (2010b) Identification of low molecular weight pyroglutamate Abeta oligomers in Alzheimer disease: a novel tool for therapy and diagnosis. J Biol Chem 285:41517-41524.

Wolfe MS (2010) Structure, mechanism and inhibition of gamma-secretase and presenilin-like proteases. Biol Chem 391:839-847.

Xu H, Sweeney D, Wang R, Thinakaran G, Lo AC, Sisodia SS, Greengard P, Gandy S (1997) Generation of Alzheimer beta-amyloid protein in the trans-Golgi network in the apparent absence of vesicle formation. Proc Natl Acad Sci U S A 94:3748-3752.

Yamaguchi H, Hirai S, Morimatsu M, Shoji M, Harigaya Y (1988a) Diffuse type of senile plaques in the brains of Alzheimer-type dementia. Acta Neuropathol 77:113-119.

Yamaguchi H, Hirai S, Morimatsu M, Shoji M, Ihara Y (1988b) A variety of cerebral amyloid deposits in the brains of the Alzheimer-type dementia demonstrated by beta protein immunostaining. Acta Neuropathol 76:541-549.

Youssef I, Florent-Bechard S, Malaplate-Armand C, Koziel V, Bihain B, Olivier JL, Leininger-Muller B, Kriem B, Oster T, Pillot T (2008) N-truncated amyloid-beta oligomers induce learning impairment and neuronal apoptosis. Neurobiol Aging 29:1319-1333.

Zanetti O, Solerte SB, Cantoni F (2009) Life expectancy in Alzheimer's disease (AD). Arch Gerontol Geriatr 49 Suppl 1:237-243.

Zhang XM, Cai Y, Xiong K, Cai H, Luo XG, Feng JC, Clough RW, Struble RG, Patrylo PR, Yan XX (2009) Beta-secretase-1 elevation in transgenic mouse models of Alzheimer's 
disease is associated with synaptic/axonal pathology and amyloidogenesis: implications for neuritic plaque development. Eur J Neurosci 30:2271-2283.

Zhang YW, Thompson R, Zhang H, Xu H (2011) APP processing in Alzheimer's disease. Mol Brain 4:3. Advance online publication. Retrieved August 21, 2011. doi: 10.1186/17566606-4-3

Zheng H, Koo EH (2011) Biology and pathophysiology of the amyloid precursor protein. Mol Neurodegener 6:27. Advance online publication. Retrieved August 21, 2011. doi: 10.1186/1750-1326-6-27.

\section{Websites}

- World Alzheimer Report 2010, Alzheimer's Disease International, UK, accessed August 2011, < http://www.alz.co.uk/research/files/WorldAlzheimerReport2010.pdf>

- German Alzheimer Report 2010, Deutsche Alzheimer Gesellschaft, Germany, accessed August 2011, < http://www.deutsche-alzheimer.de>

- Alzheimer's Association Lebanon, 2010, Beirut, accessed August 2011, < http://www.alzlebanon.org>

- Department of molecular genetics, 2011, University of Antwerp, Belgium, accessed August 2011, < http://www.molgen.ua.ac.be/ADMutations/> 


\section{Curriculum Vitae}

\section{Personal Data}

Name: $\quad$ Sadim Jawhar

Date and place of birth: $\quad$ October $25^{\text {th }}, 1984$. Al-Bass, Lebanon

Address: $\quad$ Maschmuehlenweg-4/163, 37073 Goettingen, Germany

E-mail: $\quad$ sadim.jawhar@mail.med.uni-goettingen.de

\section{Education}

4/2009-Date

PhD, University of Goettingen, International

Max-Plank Research School, Neuroscience Program

9/2007-3/2009

M.Sc, University of Goettingen, International

Max-Plank Research School, Neuroscience Program

9/2001-2/2006

B.Sc, Qatar University, Biomedical Sciences

\section{Awards and Scholarships}

2010-2012: GGNB Excellence PhD Stipend

9/2009: Golden Award for oral presentation for an excellent first year student presentation, NEURAD summer school-Lille (France)

2007-2008: Stipend of the excellence foundation for the promotion of the Max Planck society 2006: Qatar University's Distinction medal 2001-2006: Stipend Qatar University 


\section{List of Publications}

\section{Original Articles}

1. Jawhar, S., Wirths, O., Schilling, S., Graubner, S., Demuth. HU., Bayer. T.A. (2011) Overexpression of glutaminyl cyclase, the enzyme responsible for pyroglutamate Abeta formation, induces behavioral deficits, and glutaminyl cyclase knock-out rescues the behavioral phenotype in 5XFAD mice. Journal of Biological Chemistry. 286:4454-4460.

2. Jawhar, S., Trawicka, A., Jenneckens, C., Bayer, T. A., and Wirths, O. (2010) Motor deficits, neuron loss, and reduced anxiety coinciding with axonal degeneration and intraneuronal Abeta aggregation in the 5XFAD mouse model of Alzheimer's disease. Neurobiology of Aging. doi.org/10.1016/j.neurobiolaging.2010.05.027

3. Wirths, O., Erck, C., , Henrik, M., Harmeier, A., Geumann, C., Jawhar, S., Kumar, S., Multhaup, G., Walter, J., Ingelsson, M., Degerman-Gunnarsson, M., Kalimo, H., Huitinga, I., Lannfelt, L., and Bayer, T. A. (2010) Identification of low molecular weight pyroglutamate Abeta oligomers in Alzheimer disease: a novel tool for therapy and diagnosis. Journal of Biological Chemistry. 285: 41517-24

4. Cotel, MC., Jawhar, S., Christensin, D., Bayer, T.A, Wirths,O. (2010) Environmental enrichment fails to rescue working memory deficits, neuron loss, and neurogenesis in APP/PS1KI mice. Neurobiology of Aging. doi: 10.1016/j.neurobiolaging.2010.02.01

5. Wirths, O., Bethge, T., Marcello, A., Harmeier, A., Jawhar, S., Lucassen, P. J., Multhaup, G., Brody, D. L.,Esparza, T., Ingelsson, M., Kalimo, H., Lannfelt, L., and Bayer, T. A. (2010) Pyroglutamate Abeta pathology in APP/PS1KI mice, sporadic and familial Alzheimer's disease cases. Journal of Neural Transmission. 117: 85-96.

\section{Review}

- Jawhar, S., Wirths, O., Bayer. T.A. (2011). Pyroglutamate Abeta a hatchet man in Alzheimer's disease. Journal of Biological Chemistry. doi:10.1074/jbc.R111.288308

\section{Book}

- Participated in writing parts of a chapter about Alzheimer's disease in an online book. Neuroscience in the $21^{\text {st }}$ Century: Problems during aging (Alzheimer's, et al.). (2011). Springer. 